Portland State University

PDXScholar

Summer 9-1-2015

\title{
Training "In a Good Way": Evaluating the Effect of a Culturally Responsive Pre-training Intervention on Learning and Motivation
}

Adam Thomas Murry

Portland State University

Follow this and additional works at: https://pdxscholar.library.pdx.edu/open_access_etds

Part of the Psychology Commons, and the Training and Development Commons Let us know how access to this document benefits you.

\section{Recommended Citation}

Murry, Adam Thomas, "Training "In a Good Way": Evaluating the Effect of a Culturally Responsive Pretraining Intervention on Learning and Motivation" (2015). Dissertations and Theses. Paper 2480.

https://doi.org/10.15760/etd.2477

This Dissertation is brought to you for free and open access. It has been accepted for inclusion in Dissertations and Theses by an authorized administrator of PDXScholar. Please contact us if we can make this document more accessible: pdxscholar@pdx.edu. 


\begin{abstract}
Training "In a Good Way":
Evaluating the Effect of a Culturally Responsive Pre-training Intervention

on Learning and Motivation
\end{abstract}

by

Adam Thomas Murry

A dissertation submitted in partial fulfillment of the requirements for the degree of

\author{
Doctor in Philosophy \\ in \\ Applied Psychology
}
Dissertation Committee:
Keith James, Chair
Donald Truxillo
Joel Steele
Cornel Pewewardy
Timothy Anderson

Portland State University

2015 
(C) 2015 Adam Thomas Murry 


\begin{abstract}
Employee Training and Development (T\&D) is a crucial component to an organization's success and its ability to remain competitive. Although researchers in the field have discovered ways to enhance the effectiveness of training programs through the design, delivery, and evaluation process, research has not provided empirically-based recommendations for how to best train individuals whose cultural backgrounds may influence receptiveness of training curriculum. This is particularly relevant for employees whose cultural groups have been historically discriminated against, where cultural norms implicit in the training design may be met with resistance on behalf of the trainees. In the field of multicultural education, an instructional approach has been suggested to overcome cultural differences between instructor, curriculum writers, and students known as culturally responsive education. I evaluated a pre-training video prime based on this approach in the context of multi-site data-use training program for Native American educational professionals. Data-use training was delivered after exposure to one of two videos that framed the objectives of data use either in a culturally responsive way or in a generic mainstream fashion. Participants filled out surveys after the video but before the training, and then again after the training. Prime type was randomly assigned by training location. I hypothesized that participants who received a culturally responsive training prime would learn more during the data-usage training than participants who did not, and that this effect would be mediated by heightened affective motivators immediately following the culturally responsive prime. None of the hypothesized pathways were supported. There was no direct effect of my prime manipulation on
\end{abstract}


Responsive Training ii

knowledge (Hypothesis 1) or skill acquisition (H2), or on pre-training motivation to learn (H3), research-related self-efficacy (H4), goal-commitment (H5), or identification with research (H6). Furthermore, there were no indirect effects of my manipulation on knowledge (H7) or skill (H8) acquisition through pre-training motivation to learn, selfefficacy, goal-commitment, or identification with research. The motivation to learn subscale for valence had a significant positive direct effect on knowledge and skill acquisition and self-efficacy significantly positively predicted skill acquisition. Goalcommitment and motivation to learn subscales for instrumentality and expectancy had a significant negative relationship with skill acquisition. Lack of support for my hypothesized pathways is explained partially by the failure of my independent variable to influence perceptions of cultural responsiveness. A manipulation check revealed that participants did not appraise my culturally responsive priming video as significantly more relevant or culturally appropriate compared to the alternative mainstream video. Findings on the positive effects of valence and self-efficacy are discussed in support of current literature. Negative effects of expectancy are discussed in terms of stereotype threat. The implications and applications for trainers and researchers in training and minority education are followed by a consideration of this study's limitations and suggestions for future research. 


\section{Acknowledgements}

I would like to acknowledge my adviser, Dr. Keith James, my dissertation committee, the Oregon Indian Education Association, and all my family and friends that supported me on my way here. In particular, I would like to acknowledge my mother, Nancy Murry, who always modeled diligence and encouraged me throughout my life and education. 


\section{TABLE OF CONTENTS}

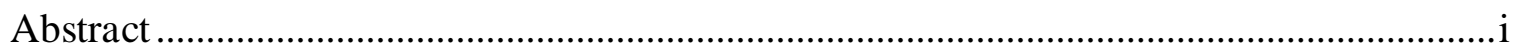

Acknowledgements ....................................................................................................... iii

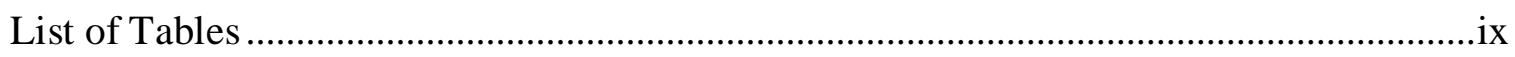

List of Figures....................................................................................................

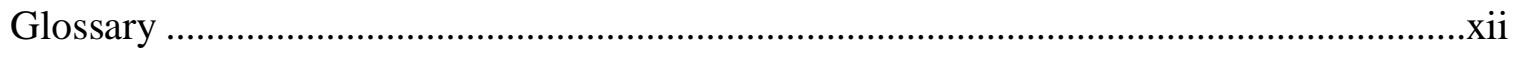

Training "In a Good Way": Evaluating the Effect of a Culturally Responsive Pre-training

Intervention on Learning and Motivation .................................................................

Culturally Responsive Education ...............................................................................

Culturally Responsive Education (CRE) and Native Americans.......................................

Study Goal \& Expectations ..................................................................................

Study Motivation ..................................................................................................

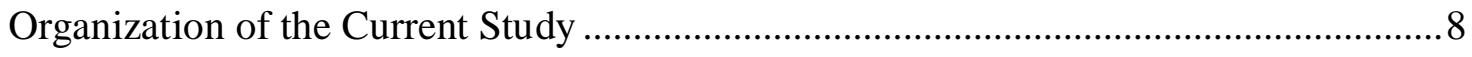

Literature Review: Training ………………………........................................

A Brief Review of Training: Past and Present ……………..........................................10

Pre-training: Training needs assessment (TNA) ………………………………….....11

During training: Instructional design and delivery ...................................................12

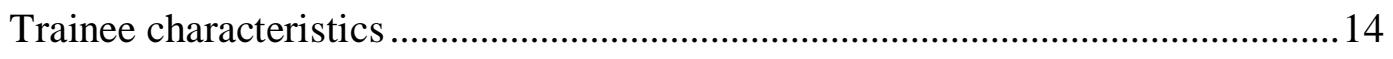

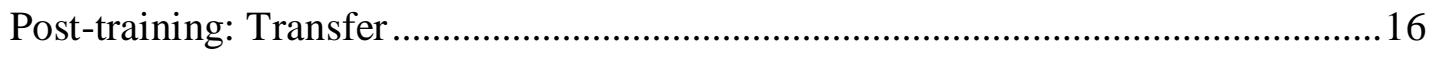

Post-training: Evaluation ...................................................................................... 17

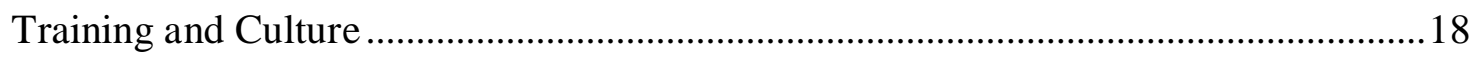

Cross-Cultural Training .......................................................................................... 19 


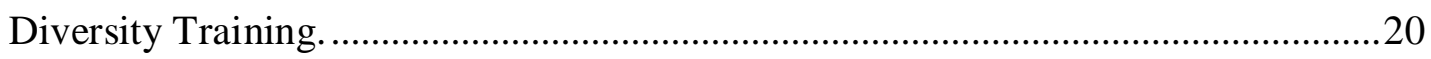

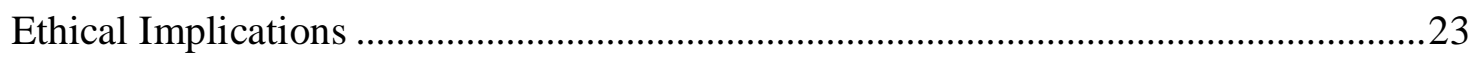

Training Racial/Ethnic/Cultural Groups .................................................................24

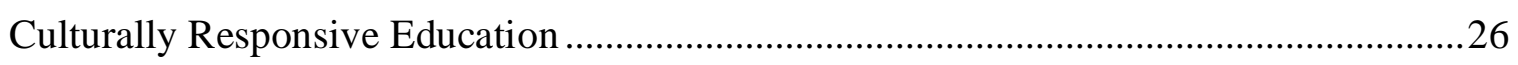

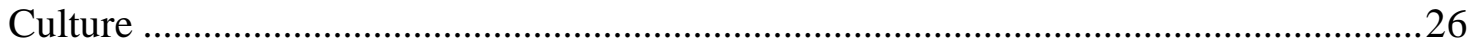

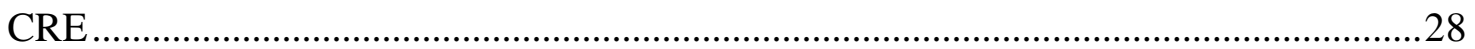

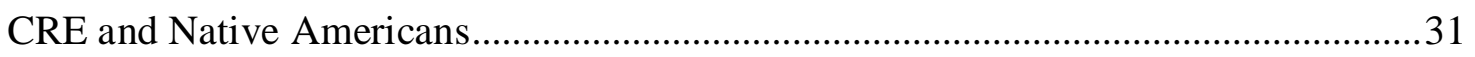

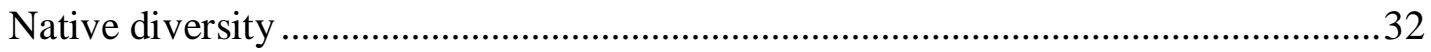

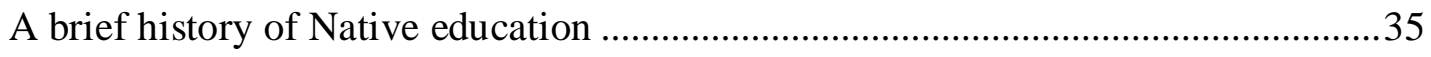

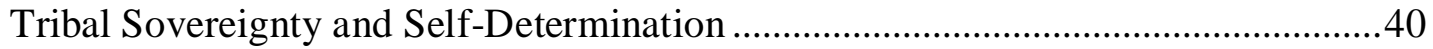

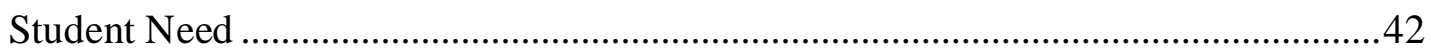

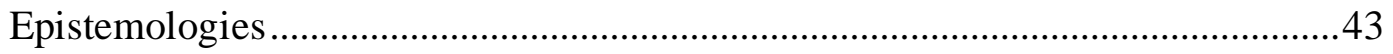

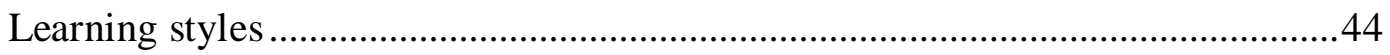

Etiquette (Communication norms) ............................................................46

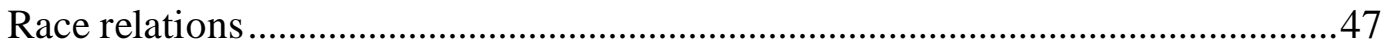

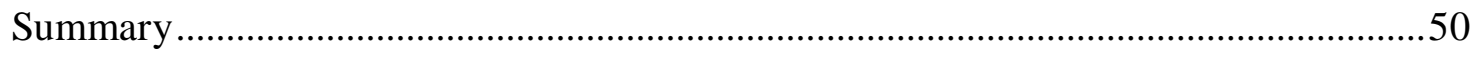

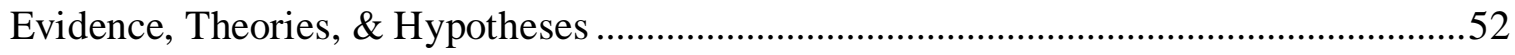

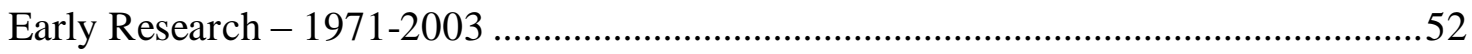

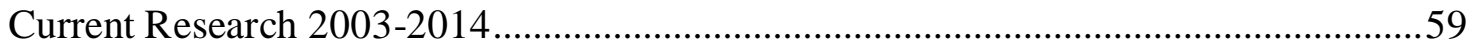

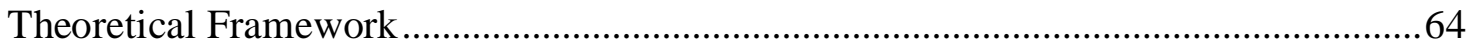

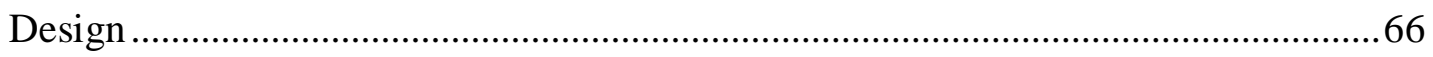

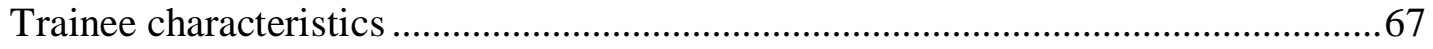




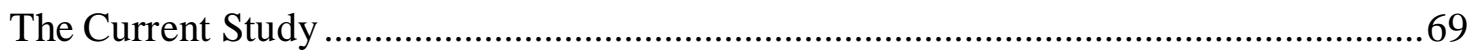

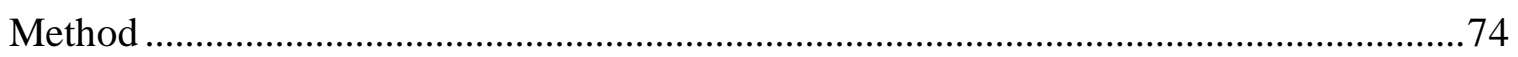

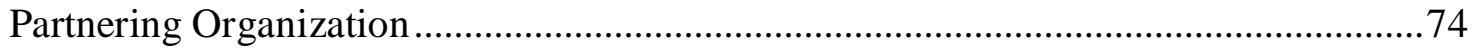

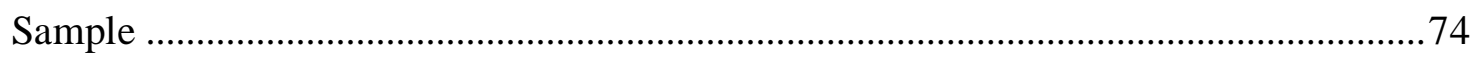

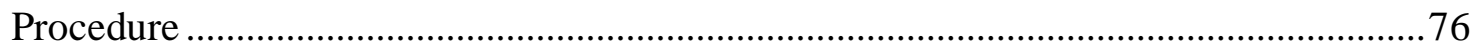

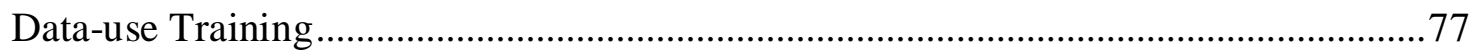

Manipulation: Culturally-responsive pre-training intervention ....................................79

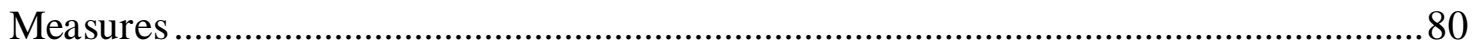

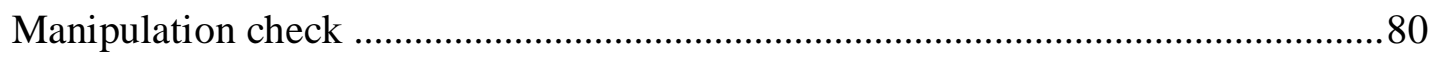

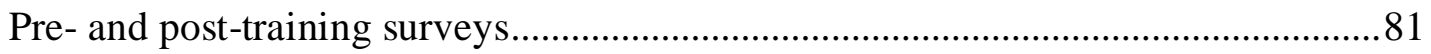

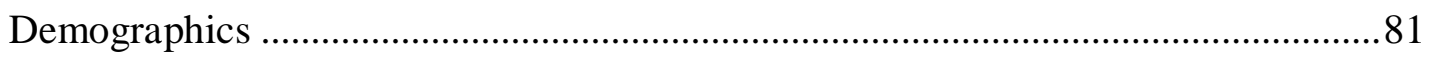

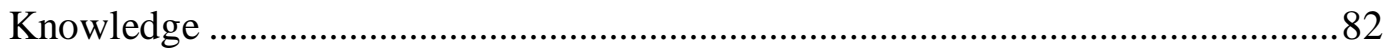

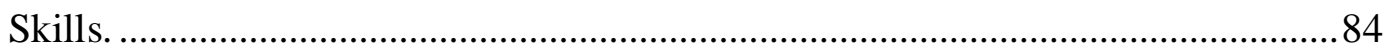

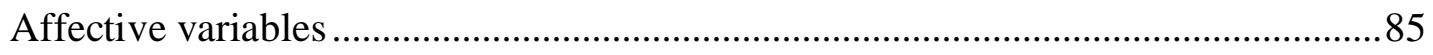

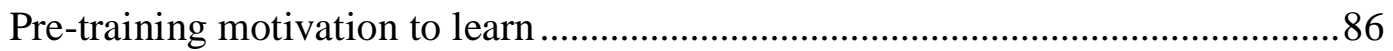

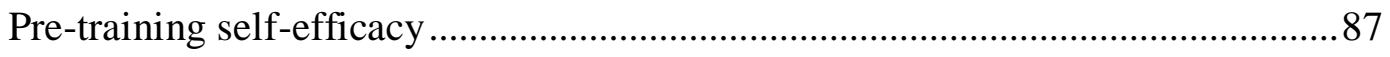

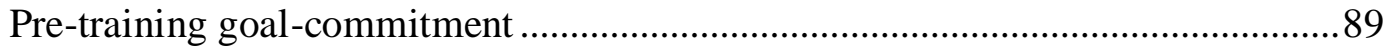

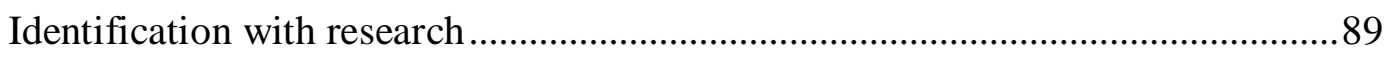

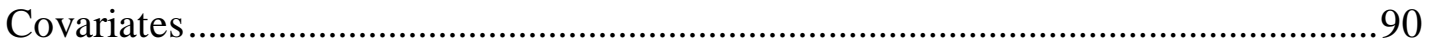

Research experience and occupational support ...............................................90

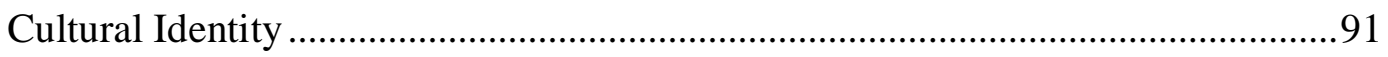

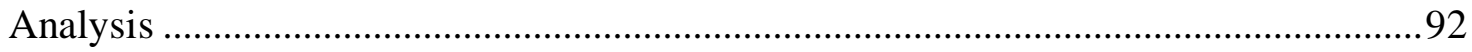




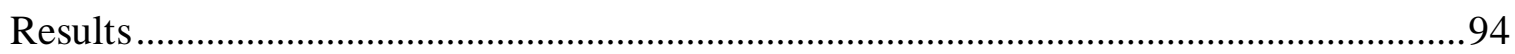

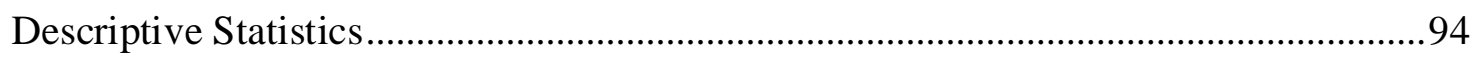

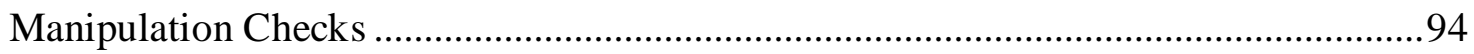

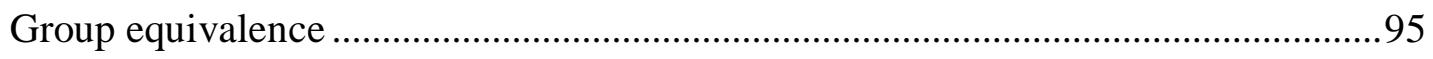

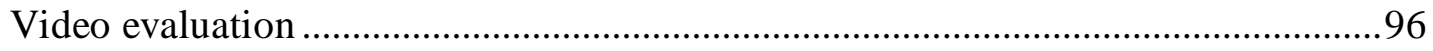

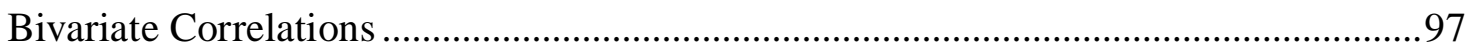

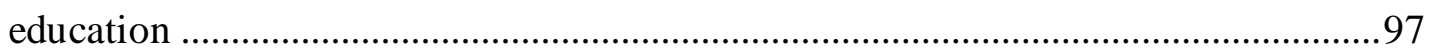

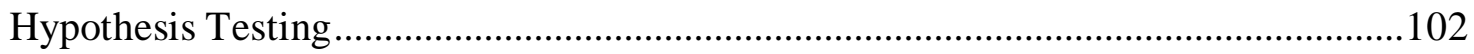

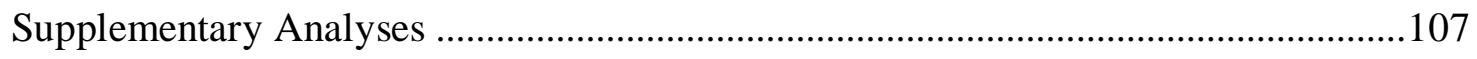

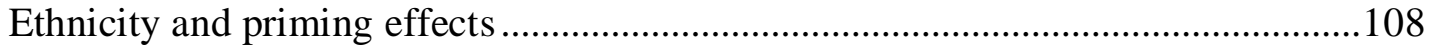

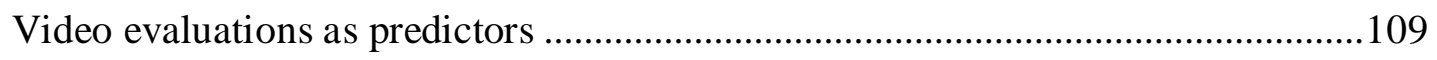

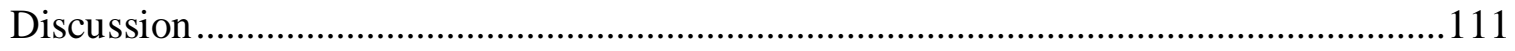

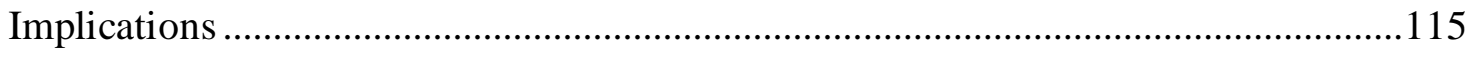

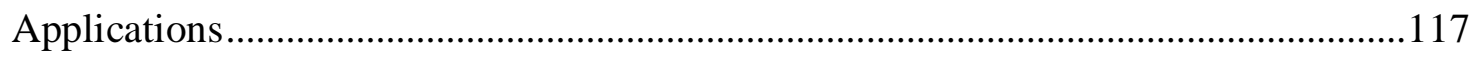

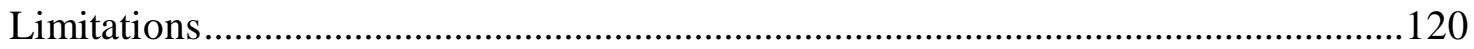

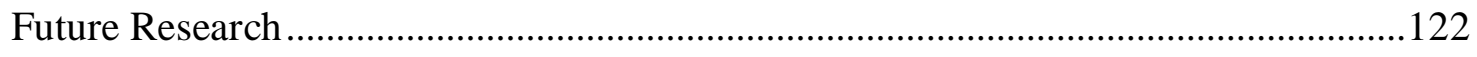

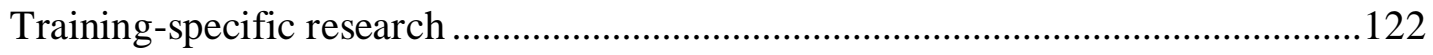

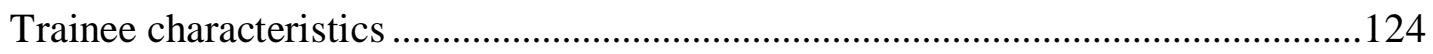

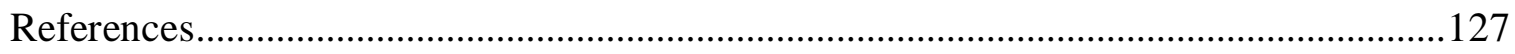

APPENDIX A: Manipulation check (video prime evaluation) ....................................171

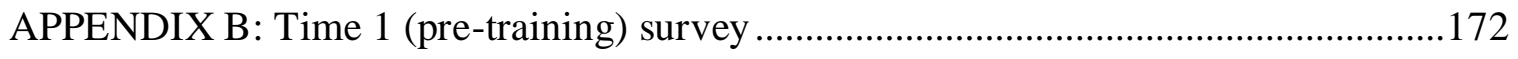

APPENDIX C: Time 2 (post-training) survey ......................................................... 181 
Responsive Training viii

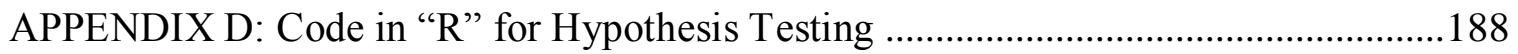




\section{List of Tables}

Table 1. Components of CRE included in CRTI ...........................................................73

Table 2. Sample characteristics by occupation and location by data collection time point.

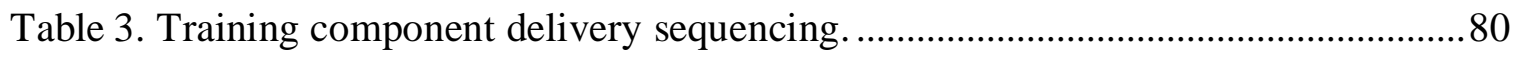

Table 4. Name and number of items for measured constructs by survey occasion. ...........82

Table 5. Ethnicity recode, sample size, and percentage of total sample .............................82

Table 6. Scoring rubric for knowledge and skill assessments.............................................83

Table 7. Knowledge and skill assessment performance statistics.......................84

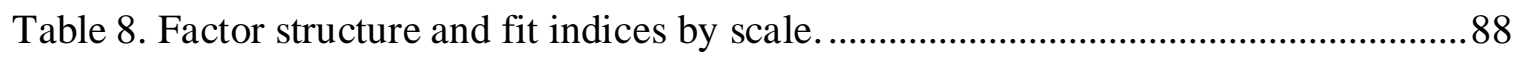

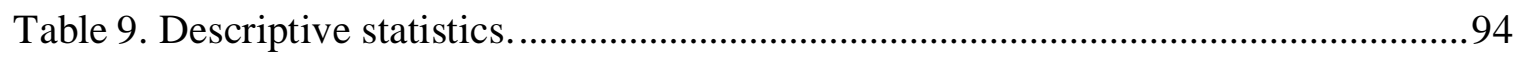

Table 10. Multivariate Analysis of Variance (MANOVA): CRTI on control variables to test group equivalence

Table 11. Correlation matrix of independent and dependent variables .............................99

Table 11b. Correlation matrix of independent and dependent variables, demographics,

and control variables continued... .............................................................................100

Table 11c. Correlation matrix of independent and dependent variables, demographics,

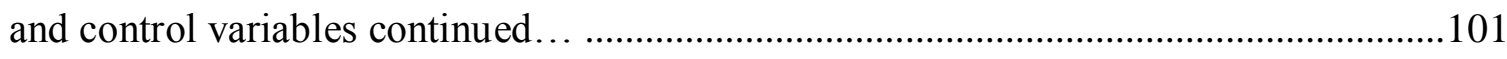

Table 12. List of statistically significant relationships between outcome variables, individual differences, and demographics..................................... 102 
Table 13. Coefficient estimates from structural equation model predicting Knowledge and Skill training outcomes from CRTI, controlling for pre-training knowledge and skills, and mediated by affective variables.

Table 14. Direct effects of CRTI on affective variables purposed to mediate its effect on learning outcomes. .104

Table 15. Coefficient estimates from structural equation model predicting Knowledge and Skill training outcomes from CRTI, controlling for pre-training knowledge and skills, and mediated by affective variables. Control variables included.. .106 Table 16. Direct effects of CRTI on affective variables purposed to mediate its effect on learning outcomes. 107 


\section{List of Figures}

Figure 1. IPO model of training evaluation.

Figure 2. Proposed model of culturally responsive pre-training intervention's effect on knowledge, skill, and affective outcomes, with affective outcomes mediating the effect of the intervention on knowledge and skills.

Figure 3. Path analysis of CRTI on knowledge and skill acquisition, mediated by motivation to learn (MTL), self-efficacy, goal-commitment, and identification with research.

Figure 4. Model 1 results: Without covariates entered.....

Figure 5. Model 2 results: With covariates entered 107

Figure 6. Qualities Associated with Traditional Knowledge and Western Science. 
Responsive Training xii

\section{Glossary}

$\mathrm{CRE}=$ Culturally Responsive Education

CRTI $=$ Culturally Responsive Training Intervention

KSAs $=$ Knowledge, skills, and abilities

KSAOs $=$ Knowledge, skills, abilities, and other characteristics

TEK $=$ Traditional Ecological Knowledge

TNA $=$ Training Needs Assessment

$\mathrm{T} \& \mathrm{D}=$ Training and Development 


\section{Training "In a Good Way": Evaluating the Effect of a Culturally Responsive Pre- training Intervention on Learning and Motivation}

The use of employee training is nearly ubiquitous among organizations. Training is valuable as years of empirical research have demonstrated its effectiveness at improving work-related behavior (e.g., performance; Aguinis \& Kraiger, 2009; Salas, Tannenbaum, Kraiger, \& Smith-Jentch, 2012; Van Iddekinge, Ferris, Perrewé, Perryman, Blass, \& Heetderks, 2009). In addition, scholars in the field of industrial-organizational psychology have argued that investments in training programs are wise because human capital is among the most accessible and malleable of organizational resources (Bourdreau \& Ramstad, 2005; Salas, Tannenbaum, Kraiger, \& Smith-Jentch, 2012). Fortunately, researchers over the past 30 years have worked to identify the critical elements of training effectiveness in regard to assessing training need, developing training objectives (i.e., KSAs and other essential characteristics), implementation (e.g., matching teaching method with task type, role modeling, error management), purposeful evaluation, training transfer, and organizational support (Arthur, Bennett, Edens, \& Bell, 2003; Cannon-Bowers, Tannenbaum, Salas \& Converse, 1991; Grossman \& Salas, 2011; Keith \& Frese, 2008; Salas, Tannenbaum, Kraiger, \& Smith-Jentch, 2012; Taylor, RussEft, \& Chan, 2005).

Although great strides have been made, societal level changes have spurned new research questions. While many questions pertain to accommodating new technologies and work environments (e.g., working remotely), one area of interest is how to accommodate cultural diversity in our theories and practices within an increasingly 
diverse workforce. In 2003, the American Psychology Association (APA) published the Guidelines on Multicultural Education, Training, Practice, and Organizational Change for Psychologists noting that "since the previous census of 1990, educational institutions, employers, government agencies, and professional and accrediting bodies are now beginning to engage in systematic efforts to become more knowledgeable, proficient, and multiculturally responsive" (p. 378, italic added).

Unfortunately with regard to the training literature, culturally responsive designs have yet to be empirically investigated. Where culture has entered the field of training, it has primarily been for the cause of training expatriates (i.e., cross-cultural training;

Littrel, Salas, Hess, Paley, \& Riedel, 2006) or improving multicultural interactions in the workplace (Chrobot-Mason \& Quinones, 2002; Kalinoski et al., 2013). This focus on training "one of ours" to work with "them" has lead the cross-cultural research away from identifying best practices for training people from other cultural groups, foreign or domestic. With the current study I attempt to address this gap by providing evidence of the efficacy of a culturally responsive training program to enhance training outcomes for culturally distinct groups.

\section{Culturally Responsive Education}

The idea to develop culturally responsive training originated from a method of instructional design called cultural responsive education (CRE) in the field of multicultural education. The method dictates updated curriculum, teacher reflexivity, the integration of culture in pedagogy, maintenance of safe classroom and school climates, and organizational support (Banks, 2007; see also Banks \& Tucker, 1998; Gay, 2000; 
2000, Nieto, 1999; Sleeter, 2011). Unfortunately, culturally responsive strategies have been infrequently applied and where they have they are exclusively focused on youth and not on adult education and training (for one exception, see Tisdell, Taylor, \& Forte, 2013). I plan to extend the research on culturally responsive education by evaluating its priming effects in an occupational training context with an adult, primarily Native American sample. From here on I will be referring to this culturally responsive prime as a Culturally Responsive Training Intervention (CRTI).

\section{Culturally Responsive Education (CRE) and Native Americans ${ }^{1}$}

CRE has been requested for Native American students in both Native and nonNative academic circles (Castagno \& Brayboy, 2008; Demmert, 2001; Gilliland, 1995; Pewewardy, 2012; Pewewardy \& Hammer, 2003), particularly within the fields of science, technology, engineering, and math (STEM; Aikenhead \& Ogawa, 2007; Allen \& Crawley, 1998; Cajete, 2000; Riggs, 2004; Semken \& Morgan, 1997). This call is critical in light of the drop-out rate of Native Americans in secondary education - they lead the nation (Chapman, Laird, Ifill, \& KewalRamani, 2011; James, Chavez, Beauvais, Edwards, \& Oetting, 1995) - and the persistent underrepresentation of Native Americans in STEM degree programs and professions (Hill, 2007; Lewis, Menzies, Najera, \& Page, 2009; Seymour, 2000). In addition to equity issues, James (2000; 2001) and colleagues have argued that underrepresentation in STEM fields leads to a limited number of tribal

\footnotetext{
${ }^{1}$ Throughout this paper I will be using the term "Native American" rather than "American Indian" to refer to descendants of indigenous nations of the United States. The label "Indian" was based on the mistaken belief by Christopher Columbus that he had landed in the East Indies.
} 
community members who are qualified to address the substantial economic, environmental, educational, and health-related issues that plague Native communities at rates often higher than other ethnic minority groups (e.g., poverty [Ogunwale, 2006]; inadequate infrastructure [Energy Information Administration, 2000]; environmental hazards [Brook, 1998; James, Hall, Hiza, Redsteer, \& Doppelt, 2008]; eligibility for special education [DOE, 2011]; exposure to violence substance abuse, and suicide [Pavkov, Leah, Fox, King, \& Cross, 2008]).

At first glance, the evidence would seem to support the use of culturally responsive practices. In Demmert's (2001) comprehensive review of the qualitative and quantitative research on CRE with Native populations, he concludes, "The preponderance of research evidence in this review shows a positive association between academic performance and the presence of Native language and cultural programs, outweighing research that shows little or no influence" (p. 12). However, a few years later Demmert, McCardle, Mele-McCarthy, and Leos (2006) report that researchers and educators developed a research blueprint that included a request for research to document whether or not culturally responsive education works (p. 102). The reason for this continued call is a lack of convincing evidence within the current body of literature, due primarily to the absence of rigorously designed experimental studies (Demmert \& Towner, 2003). As it was stated in a review by Castagno and Brayboy (2008), while "there is a plethora of scholarship consisting of case studies, program descriptions, and anecdotal calls for [culturally responsive schooling], ...many have noted that the causal links in this work 
are weak and that very few studies make strong claims about how students' academic performance is affected by efforts at [culturally responsive schooling]” (p. 982).

\section{Study Goal \& Expectations}

The goal is to quantitatively evaluate the effect of culturally responsive pretraining interventions on learning outcomes for Native American participants and identify the mechanisms through which it affects learner performance. While the mechanisms through CRE exerts if effects on performance have not yet been rigorously tested, an assortment of qualitative studies and program evaluations suggest that when teachers and curriculum designers integrate their student population's culture into the learning environment, the following affective motivators are increased: a) students' attitudes toward school, b) motivation to learn, c) self-efficacy to succeed, d) identification with the field, and e) academic goal commitment increase. Therefore, I predict that participants who receive CRTI will perform better than those who do not and that improved performance will be mediated by CRTI's effect on the above-mentioned affective motivators within a training context.

\section{Study Motivation}

This project is motivated by practical and theoretical intentions: to equip local educators with knowledge, skills, and motivation to use data to improve Native education and to expand models of training effectiveness by considering trainees' cultures in the training design and delivery. In terms of the practical contribution, I will create and provide a much-needed service (i.e., training) for Oregon-based Native community educators. According to the most recent data, only 52\% of Oregon's Native American 
high school students will graduate on time with a regular diploma (compared to $70 \%$ for Whites; Miles, 2012). In some counties up to $80 \%$ of Native student will not graduate (Curry-Stevens, Cross-Hemmer, \& Coalition of Communities of Color, 2011). Of the students that graduate, college readiness scores across four domains (i.e., English, math, science, and reading) revealed that only $8 \%$ of Oregon's Native students were prepared for college in 2013 (compared to 36\% for Whites; ACT Inc., 2013). The training provided in this study will focus on the use and interpretation of data for educational improvement objectives among Native American educators associated with the non-profit Oregon Indian Education Association (OIEA). OIEA works to enhance Native students' adjustment to and transition through school and higher education. The motivation to offer a relevant training of practical value to an organization working for the betterment of a marginalized community includes a social justice component.

It terms of the second intention to expand research regarding training effectiveness, this study will test the effect of culturally responsive pre-training intervention on trainee performance. This study addresses the above-mentioned gaps: 1) the training literature lacks empirically-based recommendations on how to train employees of diverse cultures, 2) culturally responsive education has not been studied with adult learners, and 3) culturally responsive education has not been rigorously tested and its mechanisms have not been identified, for minority populations in general and for Native Americans in particular. Should CRTI produce positive effects on learning performance and motivation, it will have implications for training and trainers at multiple levels. 
For training designers, evaluators and researchers, evidence for the effectiveness of CRTI would imply that the culture(s) of the trainee population should be considered in the design and evaluation of training effectiveness for multicultural employees. Training designers, especially for organizations that employ across culturally diverse territories, might consider CRTI when training and staff development evaluations demonstrate discrepancies in training performance and/or transfer based on employee culture, race or ethnicity, or across locations that overlap with geographically-based cultural communities. In this case, trainee culture would serve as a moderator of training effectiveness. If CRTI was shown to be more effective with particular trainees, then including trainee culture in the person analysis of a needs assessment could help select employees for training that match their cultural/value orientations (should such training be available).

For organizations, evidence for the effectiveness of CRTI has implications for employee development initiatives and the definition of organizational culture. On the surface, the appeal of CRTI is its potential to increase employee learning and transfer (i.e., improved performance) to enhance organizational production and profit. However, organizations may have more to gain from CRTI than the short-term return on investment per unit increase of on-the-job performance. Training researchers have noted the importance of the degree of similarity of training to the work environment as an important influence on transfer (Holton \& Baldwin, 2003; Holton, Bates, \& Ruona, 2000). Similar to organizational justice perceptions, CRTI may symbolize organizational concern and investment in their employees, heightening the desire for reciprocity and 
organizational commitment (Colquitt et al., 2013). Organizational commitment has been shown to decrease turnover and withdrawal intentions as well as increase attendance, performance, and organizational citizenship behaviors (Meyers, Stanley, Hercovitch, \& Topolnytsky, 2002).

For society, the results of this study could identify an important moderator of learning and performance. It may be the case that learners put forth more effort when the knowledge and skills being taught have realistic implications for themselves and similar others. To attain national-level goals, such as the need to equip all citizens with science and math skills at a level of proficiency that would prepare to contribute successfully in the workforce, CRTI suggests that societal goals be conceptualized in ways that make sense to its sub-populations' members. Empirical evidence for CRTI supports its use as a means of addressing a critical social issue in the U.S., i.e. how to educate its citizens.

\section{Organization of the Current Study}

The next three sections contain my review of the literature on each of the focal areas of this study. First, the history and importance of training and development are outlined, as well as the current knowledge regarding best practices in general and as they relate to culture. Second, I review the concept of culturally responsive education and its significance for Native Americans. Third, I review the evidence in support of culturally responsive education as an instructional design method for Native Americans and other indigenous learners. Each section delineates the research gaps addressed by this study which are summarized at the end of the review. Research questions and hypotheses are followed by the method, expected results, and a discussion of the expected results. 


\section{Literature Review: Training}

This section has two objectives: first, to present the history, importance, and currently-named best practices for training in general as a foundation for this study. It is important to state at the outset that there is scholarly consensus that training produces learning and behavioral change when research recommendations are utilized (Aguinis \& Kraiger, 2009; Grossman \& Salas, 2011; Salas, Tannenbaum, Kraiger, \& Smith-Jentsch, 2012). This study does not challenge nor attempt to clarify past studies; instead, it seeks to build on training methods that have been established in psychological research.

The second objective is to discuss the important but neglected concept of trainee culture within the training design and implementation. It should be noted that by "trainee culture" I do not mean a trainee's organizational culture that supports or hinders training transfer, otherwise known as one's continuous learning culture (Tracey, Tannenbaum, \& Kavanagh, 1995). I instead refer to the cultural memberships of the trainee that evolved outside of the work environment and encompass multiple domains of life (e.g., family dynamics, values, role expectations); whether geographically, historically, nationally, or ethnically based (Hofstede, 1980). This study aims to answer the following questions: Does training guided by current evidence-based recommendations work more effectively by incorporating trainee culture into the design and delivery? If so, through what mechanisms does it have an effect? 


\section{A Brief Review of Training: Past and Present}

Training has been defined as the intentional and planned effort to equip employees with the knowledge, skills, and attitudes necessary to improve or at least adequately perform the duties of their current or future job (Noe, 2008). Scholars have pointed out that investment in training makes sense because, of all of the organizational resources (e.g., access to raw materials, technology, or the state of the economy), human resources are the least resistant to change and the most accessible to organizational influence (Boudreau \& Ramstad, 2005). Further, socio-technological changes over the past few decades (e.g., consumer access to product/price comparisons via the Internet) have either downplayed traditional advantages in the marketplace or heightened the importance of employee-based solutions (e.g., cross-cultural communication, and employee adaptability or meta-skill development; Tannenbaum, 2002).

Only four decades ago, training knowledge had been described as a "dismal picture" (Campbell, 1971), where theoretical contribution and methodological rigor were "largely dormant" (Kraiger, 2002a). In the 1980's, major advancements were made in conducting training and development (T\&D) needs assessments (Goldstein, 1980; 1986), integrating theories of motivation and organizational support (Noe, 1986), learning and transfer (Baldwin \& Ford, 1988), and information processing (Howell \& Cooke, 1989). In response to Campbell's 1971 review, Salas and Cannon-Bowers (2001) stated that, "the past 30 years have also witnessed tremendous growth in training research... [with] nothing less than an explosion in training-related research in the past 10 years" (p. 472). As it currently stands, pioneers in the training field assert with confidence that training 
works when it is properly designed (Salas, Tannenbaum, Kraiger, \& Smith-Jentsch, 2012).

Increases in organizational investment in training have occurred in line with advancements in training knowledge. For instance, in Latham's 1988 article in the Annual Review of Psychology, he described employee training as an industry that "is now a multibillion dollar activity" (p.548). Tannenbaum and Yukl (1992) reported expenditures on formal training had reached $\$ 30$ billion annually by the time they revisited the training literature. Twenty years later, this number had increased to $\$ 164.2$ billion (ASTD, 2013) with over $\$ 70$ billion spent on corporate-level leadership development alone (Bersin, 2014). The enormity of this growing investment emphasizes the importance of designing training programs with empirically tested methods. Fortunately, the research knowledge on training best practices is fairly extensive. The most common and intuitive way to present research findings on training and development is in its order of implementation, or what has been referred to as the instructional systems design model (Aguinis \& Kraiger, 2009; Goldstein \& Ford, 2002; Salas, Tannenbaum, Kraiger, \& Smith-Jentsch, 2012; Tannenbaum \& Yukl, 1992; Wexley, 1984). This sequential approach moves chronologically from needs assessment (i.e., pre-training), to instructional design and delivery (i.e., during training), to transfer and evaluation (i.e., post-training).

Pre-training: Training needs assessment (TNA). Training programs are useful because they impact thoughts, behaviors, and attitudes that matter on the job (e.g., safety rules, procedures, compliance) and advance some organizational goal (e.g., safer workers, 
fewer injuries, lower liability). TNA is the systematic process of translating work and worker requirements into training objectives and evaluation criteria (Goldstein \& Ford, 2002). Training needs assessments focus primarily on three levels; the organization, the task, and the person (Brown \& Sitzmann, 2011; McGehee \& Thayer, 1961). An organizational analysis assesses the contextual constraints and supports of the training. These include whether training is the appropriate strategy to advance organizational goals or address organizational problems, whether there is buy-in from stakeholders at multiple levels of management/labor representatives, the condition of the transfer climate, and availability of other resources (e.g., facilities). Task analysis involves an extensive description of a job's requirements through following two main steps: 1) develop actionable statements that outline the job's important tasks, and 2) identify the knowledge, skills, abilities, and other characteristics (KSAOs) necessary to complete those tasks. Finally, person analysis identifies who needs training and the KSAOs on which they need to be trained. Assessment across all three levels provides the purpose and goal of the training, the specific areas that require training interventions, what variables to measure to determine effectiveness, and an idea of who would benefit from which type of training.

During training: Instructional design and delivery. Identifying the knowledge, skills, and abilities required for a task on the job is altogether different than facilitating a learning experience that adequately delivers those KSAOs in a training setting. Salas, Tannenbaum, Kraiger, and Smith-Jentsch, (2012) argue that a thorough training does four things: 1) conveys information that needs to be learned, 2) demonstrates desired behavior, 
cognition, and attitudes, 3) creates the opportunity to practice newly taught behaviors/cognitions, and 4) provides feedback for improvement purposes (p. 86).

This outline of effective training is supported by the research. For instance, conveying information about what needs to be learned is important for directing trainee attention and aiding the development of mental models (Cannon-Bowers, Rhodenizer, Salas, \& Bowers, 1998). Also, in a meta-analysis on training methods, lecture and discussion predicted learning across multiple skill domains (Arthur, Edens, Bennet, \& Bell, 2003). In a meta-analysis on behavioral modeling, it was found that demonstrating the desired behavior produced positive changes in procedural knowledge and job-related behavior (i.e., transfer; Taylor, Russ-Eft, \& Chan, 2005). The opportunity to practice is fundamental to developing expertise (Ericsson, Krampe, \& Tesch-Romer, 1993), leads to deeper comprehension of the material (Anderson, 1996), and prevents skill-decay (Arthur, Bennett, Stanush, \& McNelly, 1998). Lastly, providing feedback that is accurate, timely, and task-oriented (rather than person-directed) improves performance while avoiding resistance or conflict (Cannon \& Witherspoon, 2005; Karl, O’Leary-Kelly, \& Martocchio, 1993; Sasson \& Austin, 2005).

Although the information-demonstration-practice-feedback formula for strong training is instructive to designers, how trainees are instructed to approach the material during practice is also important to training effectiveness. One example is how exploration and performance errors are framed. In a meta-analysis on error management training, Keith and Frese (2008) compared training conditions where exploration and errors were encouraged, only exploration was encouraged, or neither exploration nor 
errors were encouraged. Training that encouraged both exploration and errors failed to produce better training test scores, but surpassed both of the other conditions in influencing on-the-job behavior post-training (i.e., transfer).

Trainee characteristics. Training researchers Raymond Noe and Jason Colquitt (2002) delineate that for training to be effective, there are four requirements of the trainees: Trainees must show up ready for the training (e.g., have basic skills), 2) be motivated to learn, 3) learn the training material, and 4) take what they have learned in training and transfer it to the job. However, just as training quality is not uniform across training situations, trainees vary in trainability, personality, motivation, and job attitudes to the benefit or the detriment of the training goals. In a meta-analytic path analysis by Colquitt, LePine, and Noe (2000), attitudes toward the training, job attitudes, and personality (e.g., conscientiousness, anxiety) predicted motivation to learn; motivation to learn and trainability (e.g., cognitive ability) predicted learning during training (e.g., skill acquisition and self-efficacy post-training); learning predicted transfer which resulted in improved job performance. One lesson that can be derived from these results is that optimal training experiences rely on a complex set of interacting variables, of which trainees will no doubt span the low and high ends of each continuum in countless combinations. Another lesson is that, to the extent possible, the closer we can bring each trainee to the positively impactful ends of these continuums (e.g., through pre-training interventions; Cannon-Bowers, Rhodenizer, Salas, Bowers, 1998), the more training will lead trainees toward organizational goals (in this case, better job performance). 
Another layer of complexity in attempting to facilitate learning is how trainees vary depending on sub-group memberships. In Colquitt, LePine, and Noe's (2000) metaanalysis, age was positively related to pre-training self-efficacy and job-involvement but negatively related to motivation to learn and declarative knowledge, controlling for other variables. This suggests that older workers might be unique in their training needs, given that despite their higher self-efficacy and career interest, there appears to be resistance to training. One possible explanation for this is that the training method interacts with the aptitude of the trainee population (i.e., an Aptitude-Treatment Interaction [ATI];

Cronbach \& Snow, 1977). In this case, older trainees would underperform younger trainees due to characteristics of the training that failed to resonate with their shared individual characteristics, rather than because of a lack of ability on the part of older workers. Callahan, Kiker, and Cross (2003) provided evidence for this possibility with a meta-analysis on instructional training methods with older learners. Self-pacing was found to explain the most variance in performance, above modeling, active participation, or lecture, although those other methods were effective too. Investigating the effect of age-set differences and error management training, Carter and Beier (2010) found a 3 way interaction between age, cognitive ability, and training method, such that older, trainees with higher cognitive ability did best when errors were encouraged whereas younger trainees with higher cognitive ability did best when errors were not encouraged. The implication is that training effectiveness can be influenced by sub-group differences. The same reasoning could be extended to other demographic groups, reiterating the need to evaluate group-based moderators of training effectiveness (Snow, 1986). 
Post-training: Transfer. The overarching motivation for sponsoring training activities stems from the belief that what is learned during the program will be carried over to another environment (e.g., the workplace) and thereby improve performance toward a larger goal (e.g., organizational-level performance; Goldstein \& Ford, 2002). The veracity of this belief, however, has only been partly substantiated. For example, Arthur, Bennett, Edens, and Bell's (2003) meta-analysis showed that while training works, its effect sizes were larger for learning measures in, or immediately after, training than for on-the-job behaviors. This well-known discrepancy between in-training behavior and behavior on the job has been named the "transfer problem" and has been the focus of much research (Baldwin \& Ford, 1988; Burke \& Hutchins, 2007; 2008; Grossman \& Salas, 2011; Michalak, 1981).

The most influential model guiding transfer research was put forth by Baldwin and Ford (1988) who outlined that transfer is most likely to occur when training design, trainee characteristics, and the work environment all support learning and retention during training, and generalization and maintenance after training. Their model continues to be a useful heuristic for organizing the research (e.g., Burke \& Hutchins, 2007; 2008; Grossman \& Salas, 2011) and each component has garnered empirical support (see metaanalyses by Blume, Ford, Baldwin, \& Huang, 2010, and Colquitt, LePine, \& Noe, 2000). I already discussed training designs (e.g., behavioral modeling, Taylor et al, 2005; errormanagement, Keith \& Frese, 2008) and trainee characteristics (e.g., motivation/cognitive ability, Colquitt, LePine, \& Noe, 2000) in the previous sections. Work environment features extend beyond the training and the individual to include the situational cues (e.g., 
supervisor and peer support, opportunity to perform) and consequences (e.g., punishments, positive and negative feedback) that shape the overall "transfer climate" (Rouiller \& Goldstein, 1993). In a meta-analysis by Blume, Ford, Baldwin, and Huang (2010), transfer climate and support both significantly predicted transfer.

Post-training: Evaluation. Evaluation is the empirical determination of a training program's "merit, worth, or significance" (Scriven, 2013, p.1) for the purpose of making decisions about continuing or modifying a program (Goldstein \& Ford, 2002). Evaluation is considered a research purpose and, as such, is not defined by any particular method (Rossi, Lipman, \& Freeman, 2004). However, for the sake of utility and general inference, recommendations for minimally acceptable designs given an evaluation's purpose have been described on a continuum from assessing proficiency (e.g., post-test only), to establishing training effects (e.g., pre- and post-tests) above and next to control groups (e.g., quasi-experimental), to fuller models of causation (e.g., experiment with random assignment; Sackett \& Mullen, 1993).

Similarly important factors of an evaluation, next to the rigor with which it is conducted, are the outcomes selected to demonstrate training success. As Kraiger (2002b) stated, "The technology of effective training is built largely on the knowledge of what and how to measure." Since the 1970's, the decision regarding which outcomes to measure has been guided by Kirkpatrick's (1976) four-level hierarchy, which argues that outcomes move progressively upward from trainees' reactions, learning, and behavior to the broader organizational results. 
Although Kirkpatrick's model has stood the test of time as a useful heuristic for guiding evaluation, it been criticized on logical and empirical grounds, primarily with regard to the dependence of the higher levels on the lower levels (Alliger \& Janak, 1989; Alliger, Tannenbaum, Bennett, Traver, \& Shotland, 1997), as well as the adequacy of its definitions (Alliger et al., 1997; Kraiger, Ford, \& Salas, 1993). Most notably, Kraiger et al. (1993) developed an expanded model of learning outcomes to specify whether learning occurs within three domains, namely, cognition, skill, and affect or motivation. Alliger and colleagues (1997) broke reactions into two parts (i.e., affective versus utility judgments) and distinguished in-training performance from knowledge and skills maintained on the job. Regardless of which taxonomy is chosen, scholarly consensus to date is to be mindful in selecting metrics that match the purpose of the evaluation (Kraiger, 2002b; Salas et al., 2012).

\section{Training and Culture}

Research on training effectiveness has come a long way since Campbell's (1971) critical review. However, one area of importance that has remained understudied is the interplay between training and trainee culture. In Brown and Sitzmann's (2011) review chapter on training in the Handbook of Industrial-Organizational Psychology, they commented: "Research on the effects of race, nationality, and ethnicity in training is relatively scarce, except in the area of diversity training... Research should examine which training methods are more effective and efficient for trainees' from particular racial and cultural groups" (p. 486). In a quantitative review of articles on protected groups (i.e., Civil Rights Act, 1964; 1991) specifically in industrial-organizational 
psychology journals, Ruggs and colleagues (2013) write, “The biggest and already-noted limitation of the research is the simple absence of it, particularly that which goes beyond Black-White comparisons but specifies race/ethnicity" (p. 43). Where race and culture issues have entered $T \& D$, it has not been with a focus on identifying best practices for training different cultural groups, but rather to aid current employees' adjustment to cultural others through two types of training: cross-cultural training and diversity training.

Cross-Cultural Training. The first type of culture-related training is crosscultural training. Developed in the 1960's following desegregation, cross-cultural training began with a focus on methods for fostering positive intercultural experiences, such as simulations, role plays, or the "cultural assimilator" (Brislin \& Pedersen, 1976; Wight, Hammons, \& Bing, 1969; Fiedler, Mitchell, \& Triandis, 1971). However, in the 19801990 's, with the burgeoning of economic globalization, cross-cultural training research shifted to focus almost exclusively on employees who were on foreign assignments (i.e., expatriates). This wave of research sought to identify the work and worker requirements (KSAOs) of international assignments with the intention to address the expensive and unacceptably common task failure and early return of expatriates due to poor psychological adjustment (Arthur \& Bennett, 1995; Bennett, Aston, Colquhoun, 2000; Black \& Mendenhall, 1990; Lievens, Harris, Van Keer, \& Bisqueret, 2003; Tung, 1981). The goal of cross-cultural training since that time has been "to increase the likelihood that an expatriate will be successful on a foreign assignment" (Littrel et al., 2006, p. 367) by 
helping her/him to 1) adjust personally, 2) perform professionally, and 3) interact interpersonally (Bennett, Aston, Colquhoun, 2000; Littrel et al., 2006).

Overall, cross-cultural training has been found to be effective. Meta-analyses show that it produces small to moderate changes in learning and performance during training (Deshpande \& Viswesvaran, 1992; see also review by Kealey \& Protheroe, 1998) as well as adjustment and on-the-job performance (Morris \& Robie, 2001). More recently, individual differences such as personality (e.g., openness to experience) and cognitive ability have been found to influence cross-cultural training's effect on intraining learning (Lievens et al., 2003), while self-efficacy mediates learning's effect on adjustment on-the-job (Osman-Gani \& Rockstuhl, 2009). Research on expatriate success factors in general has provided additional foci for training, including family adjustment (Palthe, 2004; Bhaskar-Shrinivas, Harrison, Shaffer, \& Luk, 2005), problem-focused coping (versus emotional-focused; Stahl \& Caligiuri, 2005), proactive networking building (versus reactive adjustment; Ren, Shaffer, Harrison, Fu, \& Fodchuk, 2014), and goal orientation and organizational support (Wang \& Takeuchi, 2007). However, despite our increase in knowledge regarding foreign assignment, cross-cultural training has not offered any empirical suggestions for how to train different racial-ethno-cultural groups.

Diversity Training. The second, and more recent, type of culture-related training is diversity training. Diversity training's are programs designed to reduce prejudice and discrimination in the workplace by raising awareness and sensitivity to diversity issues and building skills to appropriately respond to cross-cultural situations (Chrobot-Mason \& Quinones, 2002; Kalinoski et al., 2013). This training sub-field has received 
considerable attention due to the fact that it has become common-place in organizational training. An estimated $67-70 \%$ of corporations now offering some form of diversity training (Bartels, Nadler, Kufahl, \& Pyatt, 2013; Esen, 2005). Another reflection of the prevalence of diversity training is the multitude of scholarly review articles that attempt to summarize the field (Bezrukova, Jehn, \& Spell, 2012, Jackson \& Joshi, 2011; Kulik \& Roberson, 2008, Paluck, 2006; Williams \& O’Reilly, 1998). Through these reviews it is possible to discern that diversity training is most likely to demonstrate an effect when it is: 1) organizationally integrated (i.e., rather than isolated events), 2) diversity-inclusive (versus specific to one type or group, e.g., religion or Muslims), 3) uses multiple instructional methods (e.g., experiential plus lecture), and 4) employs strong evaluative methods (e.g., pre-post designs with control group; Bezrukova, Jehn, \& Spell, 2012). Unfortunately, similar to cross-cultural training (Black \& Mendenhal, 1990; Kealey \& Protheroe, 1998), a general lack of rigorous, theory-driven designs has made it difficult to explain inconsistent results or to draw conclusions about when and how different diversity training designs work best (Bezrukova, Jehn, \& Spell, 2012; Kulik, 2014). A recent meta-analysis by Kalinoski and colleagues (2013) helped to fill this gap by 1) demonstrating the moderate to large effect of diversity training on cognitive, attitudinal, and behavioral outcomes, controlling for study artifacts, and 2) identifying conditions where diversity training had the greatest effects. For example, for affective outcomes, training that includes experiential learning, with an instructor, for four hours or longer outperforms passive, computer-based, shorter training. Some recent training studies that have utilized sound methods and theory have shown effectiveness in reducing 
sexist attitudes (Ehrke, Berthold, \& Steffens, 2014), increasing sexual orientationsupportive behaviors toward lesbian, gay, bi-sexual, and transgender populations (Madera, King, \& Hebl, 2013), and lowering perceptions of discrimination among minority employees (King, Dawson, Kravitz, \& Gulick, 2012).

While diversity training is important for organizational morale (Reynolds, Rahman, \& Bradetich, 2013), legal (e.g., Equal Employment Opportunities Commission requirements), and ethical reasons (Jones, King, Nelson, Geller, \& Bowes-Sperry, 2013), it offers little empirical information on how to best train participants from different racial/ethnic/cultural groups due to its focus on improving interactions by reducing prejudice and discrimination. There are two exceptions to this statement. In a review article arguing for the use of training needs assessments in the development of diversity training programs, Roberson, Kulik, and Pepper (2003) discuss two design decisions that are relevant for training other-cultural groups. These include whether or not to host training sessions with homogenous or heterogeneous groups, and whether or not to have trainers of the same demography as their participants (pp. 159-165). The available evidence being inconclusive, they argue that decisions about group composition and matched trainer-trainee demography (when controllable) should be guided by what benefits learning as identified through the needs assessment. For instance, if similarity provides a context of psychological safety (e.g., avoiding tokenism) or if a trainer's credibility is suspect, then a homogenous group with a demographically matched trainer would be ideal. 


\section{Ethical Implications}

The lack of investigation on ethno-cultural experiences in training is peculiar given the desegregation of the workplace (Civil Right Act, 1964), the increasing percentage of non-White citizens in the U.S. over the past 20 years (see Johnston, 1991 compared to Hobbs \& Stoops, 2002), and the globalization of the marketplace (Herman, 1999). However, the gap has ethical implications as well, both for psychologists and the organizations they serve.

For psychologists, the APA's Guidelines on Multicultural Education, Training, Practice, and Organizational Change for Psychologists state that "systematic efforts to become more knowledgeable, proficient, and multiculturally responsive" are based in our ethical principles "to be competent to work with a variety of populations (Principle A), to respect others' rights (Principle D), to be concerned to not harm others (Principle E), and to contribute to social ju stice (Principle F)" (p. 378, 379). With regard to industrialorganizational psychology in particular, Ruggs and colleagues (2013) point out the following:

"In the past 20 years, 19 articles in the 7 identified I-O [industrial-organizational psychology] journals have focused on racial discrimination and/or diversity for groups other than Black employees... the most consistent theme across the studies we reviewed is that employment discrimination is prevalent for minority groups other than Blacks and that such discrimination and bias is systemic across the employment cycle" (p. 41). 
For organizations, since training opportunities can be considered part of the employment cycle, particularly with regard to skill development and advancement opportunities (Goldstein \& Ford, 2002), research on what types of training is most effective for different cultural groups is important beyond organizational efficiency. As Princeton sociologists and discrimination experts Devah Pager and Hana Shepherd (2008) explain, the distinction between differential treatment and disparate impact is that "[t]he second component of this definition broadens [discrimination's] scope to include decisions and processes that may not themselves have any explicit racial content but that have the consequence of producing or reinforcing racial disadvantage" (p. 2). If training can be designed in a way that facilitates learning and performance better for particular cultural groups, the increase in qualifications for those groups might also help to address the underrepresentation of minorities in middle and upper-level positions, along with the implicated wage and income disparities (DOL, 2011; James, 2000).

\section{Training Racial/Ethnic/Cultural Groups}

Professional interactions with different cultural groups are increasing with demographic, legal, technological, and economic trends both locally and globally. Researchers have acknowledged that diversity must be managed effectively to make manifest its potential benefits (e.g., increased creativity and innovation, enhanced problem solving) rather than its potential liabilities (e.g., increased conflict and miscommunication, poor performance; Jackson, Joshi, \& Erhardt, 2003; Taylor, James, \& Murry, 2012). In addition to helping workers on foreign assignments or in diverse organizations adjust to other cultures, I propose that training methods that utilize trainee 
culture are also needed for training employees of a particular cultural group. This is especially true since ethno-cultural communities tend to be clustered geographically (Brewer \& Suchan, 2001) and employees tend to remain at organizations where there are similar others (Schneider, 1987). The combined effect of these two factors raises the odds that trainees within a particular location will consist of only a few cultural communities, especially in non-metropolitan locations.

In the following section I describe what has been called CRE, an instructional design that is gaining promise in the field of education. The design is unique in that it seeks to utilize student's home cultures to enhance motivation and learning in school; an adapted version of which I aim to test in an occupational adult training. My discussion of CRE will focus on its development within one cultural group, as a context for the actual training and to give visibility to an often understudied group of employees, i.e., Native Americans. 


\section{Culturally Responsive Education}

My review of the training literature reveals that a missing element in our training knowledge is how to maximize training effectiveness for people from different cultural groups. In this section I will review how this issue has been understood and responded to in the field of education. First I begin by defining culture and what has come to be known as CRE. Second, I describe the significance and evolution of this concept for Native Americans as a distinct ethno-cultural group. My focus on Native Americans is motivated by two factors, a) Native Americans have a unique legal and political history and experience with education that makes the appeal of culturally responsive education particularly relevant, and b) despite the fact that Native Americans are the host people of this continent they are almost completely invisible in the industrial-organizational psychology literature. In the next section, I review the evidence for this approach and extrapolate the lessons learned to make predictions for adult training using theories of motivation and learning from the training literature.

\section{Culture}

In an academic sense culture is a complex construct. Scholars have debated its exact meaning for over a century and are still arguing over the specifics (Jahoda, 2012). Anthropologists generally agree that culture is an inherently shared phenomenon that is transmitted symbolically and inter-generationally, is stable and yet dynamic and adaptive to physical/social environmental change (Ferraro, 2001). With this broad definition, "culture" can develop within any form of social organization or interacting category of people that is sustained over time, whether its boundaries are organizational/co-workers, 
national/fellow-citizens, geographical/indigenous tribesman, or demographical/sub-group members (Hofstede, 1998). Culture is important because it influences peoples' thoughts, feelings, and actions in ways that might affect how they respond to a particular training. In his influential article, Geert Hofstede (1980) argued that human action was predictable given knowledge of one's situational demands and mental programming. Mental programs, he explains, are the product of biological (i.e., universal), collective (i.e., shared), and idiosyncratic (i.e., individual) influences. He writes, "My personal definition is that culture is the collective programming of the human mind that distinguishes the members of one human group from those of another. Culture, in this sense, is a system of collectively held values" or broad tendencies "to prefer certain states of affairs over others" (p. 24, 19). According to this definition, culture a) provides a sense of group identity, b) contextualizes appropriate goals (i.e., individual-level values), and c) evaluates and motivates behavior (i.e., along with context, universal and individual drivers).

As trainers we provide a context for behavior that is designed to increase knowledge or change attitudes and behavior at the individual level without considering how cultural context may influence the delivery or the reception of the material. This is problematic since cultural variables have been shown to be of consequence. For example, organizational cultural values have been shown to predict training transfer (Simosi, 2012) and national culture values have a demonstrated effect on multiple attitudinal, behavior, and performance outcomes (see meta-analyses by Jaramillo, Mulki, \& Marshall, 2005; Taras, Kirkman, \& Steel, 2010). In addition, both organizational and national cultural 
values interact with individual-level values (i.e., normative organizational commitment, self-efficacy) to predict turnover and training transfer (Simosi \& Xenikou, 2010; Wasti, 2003a; 2003b). With the knowledge that organizational and national culture (measured almost exclusively through values) impacts training and performance on the job, it is reasonable to expect that ethnic culture, or the collective mental programming shared by people of common ancestry, history, and tradition, would also impact learning and transfer from training (Roberson, Kulik, \& Pepper, 2009). Due to the fact that this issue has not been explored with regard to training effectiveness, it is necessary to look to the education literature to see how culture can affect learning.

\section{CRE}

The idea for CRE for minority ethnic populations in general was born in the socio-political upheavals of the 1960's Civil Rights Movement. As long-time researcher and advocate of CRE Geneva Gay (1983) explains, civil rights demands for inclusion and accurate representation of marginalized groups led to critical research about the methods and theories of education. Gay (1983) recalls, "Many educators and social scientists who had endorsed the deprivation theory that undergirded compensatory education in the 1950's began to rethink their premises" (p. 561). Research shifted from explaining grouplevel differences in performance with individual or ethno-cultural inferiority (e.g., Jensen, 1969; Bloom, Davis, \& Hess, 1965) and has since evaluated the role of the classroom environment, such as the effect of preferential teacher behavior on student performance (Rosenthal \& Jacobson, 1968; Südkamp, Kaiser, \& Möller, 2012) and content analyses of instructional materials (Sleeter, 2011). A noteworthy perspective was shared by Sleeter 
(2011) after a review of several content analyses on instructional materials, that given the dominance of Euro-American worldviews and narratives in textbooks throughout K-12 and professional schools, mainstream curricula could be viewed as "Euro-American ethnic studies" (p. vii). The implication being that mainstream education is already culturally responsive, albeit for some White Americans.

In the late 1980's-early 1990's, the research program of Gloria Ladson-Billings $(1989 ; 1990 ; 1994)$ investigated the rationales and methods of teachers that were especially effective with African American youth (according to their grades and reports from their parents). Together they distilled the essential components of their approach and coined the term culturally relevant pedagogy (Young, 2010). Rather than define a particular style, she explained that culturally relevant pedagogy rests on three criteria: a) academic success of students, b) development/maintenance of cultural competence, and c) development of a critical consciousness for active citizenship (Ladson-Billings, 1995b).

At the same time other scholars were developing similar approaches, but even now agreement over what to call it has not been settled.. The concept has worked through many titles over the years with different formulations (e.g., multiethnic, multicultural, sociocultural, culturally congruent, culturally sensitive, culturally tailored and culturally relevant), different foci (e.g., curriculum, teaching, pedagogy, or schooling), and with different populations (Brown-Jeffery \& Copper, 2011; Mohatt \& Erickson, 1981; Ladson-Billings, 1995a; Nieto, 1999; Villegas \& Lucas, 2002). Gay (2000; 2002), writing on culturally responsive teaching in particular, defined the practice and its rationale as, 
“[U]sing the cultural characteristics, experiences, and perspectives of ethnically diverse students as conduits for teaching them more effectively. It is based on the assumption that when academic knowledge and skills are situated within the lived experiences and frames of reference of students, they are more personally meaningful, have higher interest appeal, and are learned more easily and thoroughly" (2002; p. 106).

Multiple scholars have offered taxonomies to outline the necessary steps to culturally responsive teaching (e.g., Howard, 2003; Villegas \& Lucas, 2002). The most popular are those of University of Washington professors James A. Banks (2007; see also Banks \& Banks, 2009; Banks \& Tucker, 1998) and Geneva Gay (2000; 2002). While they differ on the order and labeling of the components, each advocates, 1) integrating content and designing curriculum that is relevant to minority groups' self-concept and community needs (e.g., accurate histories and current scholarship guided by community values and practical needs); 2) teaching students and teachers about the assumptions and historically embedded nature of knowledge with comparisons across cultures; 3) utilizing communication, pedagogy, and learning styles from the students' home culture to facilitate learning in the classroom context; and 4) building a safe, non-discriminating learning environment for participation of diverse students and their families. Banks (2007) adds a fifth element regarding empowering the school's overall culture and structure to support classroom activities of this nature.

Despite the variety of titles and formulations, the common element of these approaches (which I refer to generally as CRE) is the goal to enhance self-efficacy and 
motivation to learn by making education safe, empowering, and personally and communally relevant (Morrison, Robbins, \& Rose, 2008; Gay, 2000). I submit that adult training would benefit from similar culturally responsive approaches and plan to evaluate a design to test the proposition. I will be using the phrase "culturally responsive" as I agree with other authors (Castgno \& Brayboy, 2008; Gay, 2000; Klump \& McNeir, 2005) that it best captures the dynamic, or changing, nature of the student-teacher relationship/instructional approach across cohorts and cultural groups.

\section{CRE and Native Americans}

Increased relevance of curriculum to pupils' lives has been argued to be crucial to educational reform for students in general, both nationally and internationally (e.g., in science education see Busch, 2005; Fusco, 2001; Osborne \& Collins, 2001). However, the enhanced cultural relevance CRE offers has a special appeal to Native Americans in that, 1) they themselves are culturally diverse, 2) education has historically not served the interests of Native communities, 3) CRE has broader implications for Tribal selfdetermination and sovereignty, and 4) it has been called for by both Native and nonNative educators who work with Native students as a promising approach to address the persistent achievement gap (Castagno \& Brayboy, 2009, Gilliland, 1995; Pewewardy, 2002; Pewewardy \& Hammer, 2003; Philips, 1976). It should be noted that although my focus for this project is Tribal nations within the United States, similar arguments, research, and educational reform is taking place for Indigenous students in other nations, such as Canada's First Nations (see Agbo, 2004; Ball, 2005; Maina, 1997), New Zealand 
Maori (see Averill et al, 2009; Savage et al., 2011), and Australian Aborigines (HicklingHudson, 2003, 2005; Santoro, 2007).

Native diversity. According to the 2011 American Community Survey, between $.9 \%-1.7 \%$ of the 309 million citizens of the United States report being Native American (i.e., between 2.9 - 5.2 million depending on whether mixed, or multi-racial, Natives are included for the higher estimate; Norris, Vines, \& Hoeffel, 2012 ${ }^{2}$ ). The label "Native American" refers to an ethnic category of people whose ancestors are indigenous to the United States. It is often used interchangeably with "American Indian" or "Indian" and placed alongside Alaskan Natives (e.g., AI/AN, see U.S. Census). I will be using Native American to refer to both except when the research is specific to one or the other. While I recognize that the term "Indian" is still in use both officially and popularly (e.g., Bureau of Indian Affairs [BIA], Indian Country Magazine), I choose to use the term Native American, or simply "Native," to avoid the somewhat antiquated misnomer.

The singular demographic category for Native Americans as an ethnic group should not imply uniformity in ethnicity or culture. On the contrary, Native Americans differ by tribe(s) as well as the degree to which one identifies with their heritage. As of 2014, there are 566 federally recognized tribes (about 229 are Alaskan Native; BIA, 2014) with an estimated 169 Native languages still in use to some extent (Siebens \& Julian, 2011). Federally recognized tribes are, “Any AI/AN, Band, Nation, Pueblo, or other organized group or community, including any Alaska Native village... acknowledged by the federal government to constitute a tribe with a government-to-

\footnotetext{
${ }^{2}$ This number includes indigenous Latin American respondents.
} 
government relationship with the United States" (US Census, 2008, p.2). Culturally speaking, while tribes that share linguistic roots and geographic, or ecological, zones tend to have much in common, even among these there is significant between-group as well as within-group variation (Waldman, 2009). For instance, in Nagel's (1994) discussion of socially constructed identity, she mentions that Native American identity has multiple layers, including;

"Subtribal (clan, lineage, traditional), tribal (ethnographic or linguistic, reservation-based, official), regional (Oklahoma, California, Alaska, Plains), supra-tribal or pan-Indian (Native American, Indian, American Indian). Which of these identities a native individual employs in social interaction depends partly on where and with whom the interaction occurs. Thus, an American Indian might be a "mixed-blood" on a reservation, from "Pine Ridge" when speaking to someone from another reservation, a "Sioux" or "Lakota" when responding to the U.S. Census, and "Native American" when interacting with non-Indians" (p.155). Moreover, individuals can vary on how much they identify with and embody their Native-ness (James, 2006). Very much due to the experience of colonization and policies directed at assimilating Native Americans into American society (described in the next section), Native identity has been described in terms of a continuum between remaining fully traditionally Native to being fully acculturated to American society. In an early study on the Menominee reservation about adaptation to culture change, Spindler and Spindler (1958) identified five different groups representing increasing levels of acculturation to American (“Western”) values: Native-oriented (i.e., traditional), peyote 
cult (i.e., Western institutions subsumed into Native context), transitional, and acculturated (broken into lower status-acculturated and elite-acculturated). La Frombroise, Trimble, and Mohatt (1990), describing a model of Native cultural identity used for clinical purposes listed the aforementioned traditional-transitional-assimilated sequence, but also distinguished categories for bicultural (equal membership/functioning in both traditional and dominant cultural settings) and marginal (neither acceptance/functioning in either cultural setting) identities. Oetting (1990-1991) and colleagues' (1998) work with the Orthogonal Cultural Identity scale demonstrated empirically that individuals can endorse more than one cultural orientation simultaneously and that one's minority and dominant cultural orientations load on different factors (see also Moran, Fleming, Somervell, \& Manson, 1999). Walters (1999), using her validated scale of Urban American Indian Identity Attitudes, furthered the discussion by showing that the degree to which one's Native identity is empowered predicts types of acculturation differently (i.e., assimilation, biculturalism, or resistance). She found a more empowered identity negatively predicted assimilation and positively predicted biculturalism and resistance.

The diversity of Native identity on both group and individual levels signals the importance of CRE and CRTI for work within as well as for Native communities. As it can be seen in the discussion above a one-size-fits-all method of instruction could be too broad even if that method were narrowed down exclusively for "Native Americans." The homogeneity/hetereogeneity of the audience could make more tailored or more general approaches more or less appropriate. If training were being held on or near a particular 
tribe's reservation a more explicit and delineated integration of that tribe's culture might be more effective. However, in diverse urban settings, where between $67-92 \%$ of Native Americans reside (i.e., depending on Native alone or mixed-race; Norris et al., 2012), a more general or pan-Indian approach may be more effective.

The diversity of Native identity also testifies to the importance of a needs assessment that considers the training target population in the development of CRTI. This is especially true as self-reported Native identification has grown exponentially over the years, even beyond tribal enrollment (Thorton, 1987). Nagel (1995) and Thornton (1997) remind us that ethnic pride movements of the 1960's and 1970's, along with the romanticizing of Native culture in popular movements and media, has led to spikes in Native self-identification in certain decades. Norton and Manson (1996) admonish researchers to qualify "Who is American Indian?” for purposes of generalization, and while they advocate the use tribal enrollment numbers, the fact that the number of federally recognized tribes has increased by over a hundred since their publication suggests that this may not be a sufficient metric.

A brief history of Native education. To understand the significance of culturally responsive approaches for Native Americans, it is necessary to situate the practice within the larger historical context. Education has earned a rather negative stigma in the Native community due to its use for purposes other than skill acquisition (James, 2001). Prior to the arrival of Spanish, Dutch, and other European colonists, education was an intergenerational and community endeavor, where information was transmitted in various 
forms (e.g., stories, songs, rituals, and mentorship) for the sake of survival and symbiotic co-existence with local ecology (Cajete, 1994; Berkes, Colding \& Folke, 2000).

Following the arrival of explorers and colonialists, education for Native Americans was usurped first by the missionaries (as early at 1568) and later by the U.S. government's boarding and public schools (Berry, 1968; DeJong, 1993). In some respect, education was requested by Natives. As legal scholar Felix S. Cohen (1945) noted in his now landmark text on Indian law,

"In payment for land ceded, and occasionally by way of compensation for other benefits or indemnification for injuries done to Indians, the Federal Government assumed extensive financial obligations to the Indian tribes... The United States agreed in treaties with most of the tribes to pay annuities in various forms: for education, blacksmiths, farmers, laborers, millers, millwrights, iron, coal, steel, salt, agricultural implements, tobacco, and transportation” (p. 44-45).

The history is long and complex and beyond the scope of this review, however, the overriding theme is that rather than provide a means of participating in the US economy, "Indian education" became an avenue to Christianize and civilize the Indigenous population by overwriting Native culture (Berry, 1968; Deyhle \& Swisher, 1997; Lomawaima, 1999; Reyhner, 1993; Szasz, 1983). For example, when Dartmouth was chartered in 1769, it was dedicated to the "education and instruction of youth of Indian tribes of this land in reading, writing, and all parts of learning which shall appear necessary and expedient for civilizing and Christianizing children of pagans" (Atkinson, 1769). Similarly in 1879, Captain Richard Henry Pratt remarked in the opening speech of 
the first government-run boarding school (i.e., Carlyle Indian School), “A great general [Philip Sheraton] has said that the only good Indian is a dead one, and that high sanction of his destruction has been an enormous factor in promoting Indian massacres. In a sense, I agree with the sentiment, but only in this: that all the Indian there is in the race should be dead. Kill the Indian in him, and save the man" (cited in Fleming, 2006).

The lesson to extract from this is that education for Native Americans had become associated with assimilation and de-identification, conformity and oppression (Deyhle \& Swicher, 1997). Methods to enhance "education" included mandates for children to leave their families for school, cutting students' hair and assigning uniforms, punishment for use of one's tribal language, frequent corporal punishment and manual labor, and regular “outings" (i.e., vacations spent in devout Christian homes); all to better interrupt the transference of Native culture and prepare students for low-end jobs (Berry, 1968; DeJong, 1993; Deyhle \& Swicher, 1997; Reyhner, 1997; Trennert, 1982, Ziibiwing Center, 2011). In 1924, the Indian Citizen Act raised attention to Native issues in Congress and the American public, motivating the Department of the Interior report The Problem of Indian Administration, otherwise known as the Merriam report after its author Lewis Merriam (Hunt, 2012).

The Merriam report heavily criticized Indian education programs for their lack of adequate funding, which resulted in sub-standard teachers, derisory assessments of student learning, child labor for school upkeep (consuming up to half of a student's day), overcrowding, the spread of preventable disease, poor nutrition, and unsanitary housing and bathroom facilities (Merriam, 1928). Notably for this project, Merriam (1928) 
defined education, "In its widest sense and include[d] not only school training for children but also activities for the training of adults to aid them in adjusting themselves to the dominant social and economic life which confronts them" (p. 9). Further, Merriam stated that, "The effort to substitute educational leadership for the more dictatorial methods now used in some places will necessitate more understanding of and sympathy for the Indian point of view. Leadership will recognize the good in the economic and social life of the Indians in their religion and ethics, and will seek to develop it and build on it rather than to crush out all that is Indian" (p. 23).

Forty years later Indian education was criticized again, this time with a focus on educational outcomes. Senator Robert Kennedy reviewed a range of indicators collected by that time (e.g., percentage of population in school, dropout rates, level of education, income, and self-efficacy in school) and concluded that the nation had failed to keep its commitments to the tribes as Natives maintained the worst statistics of any other group (see also the Havighurst Report, 1970). The 1969 “Kennedy Report," Indian Education: A National Tragedy - A National Challenge, made recommendations, among others related to health and administration, for the development of bi-cultural or "culturally sensitive" materials for education (p. 116-117, 121-122). The report led to the Indian Education Act of 1972, which provided funds to public schools with Native students, recognizing "that American Indians have unique, educational and culturally related academic needs and distinct language and cultural needs" (OESE, 2005). Part C of this act referred specifically to adult education. The importance of this mission was reaffirmed with amendments and continued funding in 1994 (public law 103-382), 1998 
(executive order 13096), 2001 (public law 107-110), 2004 (executive order 13336), and 2011 (executive order 13592). Unfortunately, despite national attention Natives still have some of the worst educational and employment outcomes of any other American minority and culturally responsive designs have yet to become the norm (Indian Nations at Risk Task Force, 1991; NIEA, n.d.; Ross et al., 2012; United States Commission on Civil Rights, 2003; see also section on Student Need below).

What is important to consider for my purposes of integrating culturally responsive approaches into adult training is how the stigma of education might carry over to other learning environments. For example, Mohatt, Thompson, Thai, and Tebes (2014) postulated that current health statistics for Native Americans (which are comparable to their negative educational statistics) are impacted by intergenerational and collectively experienced traumas. While they include structural inequalities that are the result of oppression, they argue it also has a continued effect through contemporary public narratives within the community. With regard to education, recent information regarding the scandalous nature of education for Native Americans no doubt heightens awareness of the fact that societal institutions have not served Native interests. Recent examples include the largest settlement case to date from the Roman Catholic church paid to Native students of boarding schools throughout the Pacific Northwest for almost ubiquitous physical and sexual abuses (Yardley, 2011) or the recently uncovered experiments in starvation in First Nations boarding schools in Canada (Mosby, 2013).

Although the discussion pertains most directly to contexts of compensatory (i.e., K-12) and higher education, such experiences have implications for employment contexts 
as well, since educational and employing institutions are presumably inter-related and complementary. In addition, while education and employment may be distinct in the mind of the average citizen, for those populations who have experienced perpetual discrimination, each is part of single sequentially interconnected system (James, 2001). This section points out how not only has the U.S. Government endorsed culturally responsive practices for Native American youth and adults, but also that culturally responsive approaches may help to fulfill a long-standing promise to integrate Natives into the competitive economy without requiring them to abandon their identity.

Tribal Sovereignty and Self-Determination. Culturally responsive approaches to education are pertinent to Native Americans apart from appealing to their sense of identity and disassociating from instructional platforms of past injustice. They are also important for recognizing tribal sovereignty and self-determination. It is important to remember that Native tribes have been recognized as independent sovereign nations within the United States since its foundation (e.g., the Treaty of Fort Pitt, 1778). However, despite their independence, policies made on behalf of Native Americans vacillate on whether to recognize their freedom or regulate their lives.

For example, the Dawes Act of 1887 (also referred to as the General Allotment Act) divided up tribal territory into individual family plots. The goal was to ward Natives away from communal living and teach them the value of individual property ownership. Native lands that fell outside of the determined need for individual allotments was deemed surplus and sold to land speculators (Rollings, 2004). After respectable participation of Natives in WWI this act was overwritten by the Indian Reorganization 
Act of 1934, which put a halt to the allotment process and returned portions of seized “surplus" land. Around 20 years later, Native land trusts were dissolved altogether for over a hundred tribes with the Termination Act of 1953 (public law 588; Walch, 1983) and those remaining on reservations were given incentives to leave with the promise of work with the Indian Relocation Act of 1956 (public law 959). Another example is the Indian Citizenship Act of 1924. Prior to the act citizenship was only awarded to Natives on a negotiated basis (e.g., by trading land, renouncing tribal citizenship, or joining the military). Following the act all Natives born within the U.S. were considered citizens; however, citizenship did not include the right to vote ${ }^{4}$ (Houghton, 1931) or freedom of religion. ${ }^{5}$

Following the civil rights movement, legislation changed for Native Americans. President Nixon retracted the termination policies of the 1950's and argued that tribes be allowed to control their own affairs while still being eligible for Federal assistance. The Self-Determination and Educational Assistance Act of 1975, public law (93-638), made it possible for Native organizations to apply for grants and contract services otherwise assumed exclusively by the government with regard to health, public safety, environmental management, and education, among others. It has been argued by Native and non-Native authors (Brayboy, 2005; Brayboy \& Castagno, 2009; Castagno \& Brayboy, 2008; Deyhle \& Swisher, 1997; Lomawaima \& McCarty, 2002) that culturally

\footnotetext{
${ }^{4}$ As of 1948 all but four states allow Native to vote, Arizona and New Mexico changed in 1948, followed by Utah in 1957, and Maine in 1967 (Rollings, 2004).

${ }^{5}$ The right for Native freedom of religion was provided by the American Indian Religious Freedom Act of 1978, in public law 95-341.
} 
responsive education is a manifestation of self-determination, in that it gives Native communities input and control over what and how education will be implemented. Should input from the affected Native communities be included into the design of employee training, the same implications for sovereignty and autonomy in CRE could be applied to the employment setting.

Student Need. The final rationale for utilizing culturally responsive approaches with Native students has come from scholastic arguments that such designs will help close the persistent achievement gap in education and employment through incorporating student characteristics. Unfortunately, since the time of Senator Kennedy's review of the educational statistics for Native American's, their position has not changed. For example, the National Indian Education Association (n.d.) keeps an updated review of national educational metrics to evaluate the status of Native American students. Native students are more likely than White students to be offered drugs and be threatened with violence at school (Robers, Kemp, \& Truman, 2013), to be suspended (OCR, 2012), to be eligible for special education, experience poverty, attend schools with lower average yearly progress or academic counseling, and are less likely than White students to have parents with a higher education, score as college-ready on high school assessments, graduate with a degree, or earn a comparable income when working in similar fields (STEM) with similar education (Ross et al., 2012). The situation has kept the question open as to what factors prevent Native students from doing well in academe.

Scholars in psychology, education, educational anthropology, and related topics have proposed that, in addition to environmental factors such as poverty and lack of 
infrastructure (see James, 2000), educational disparities are the result of cultural differences between student home and school cultures. The proposition has been named "Cultural Difference Theory" and grew to replace the cultural "deficit" theories of the 1950's and 1960's (Banks, 2013; Lemke, 2001; Schmeichel, 2012; Villegas, 1991). Although there are different ways to divide the cultural differences literature comparing Native Americans and mainstream schooling (see Castagno \& Brayboy, 2008), arguments generally fall into the realms of epistemologies, learning styles, and etiquette (i.e., communication styles), or how Natives think, learn and act. This section is not intended to be exhaustive, but still provide a cursory review.

Epistemologies. Epistemology refers to the methods and limits of creating knowledge. In comparing and contrasting ways of knowing between Native societies and mainstream institutions, much of the work in this area has been done within the science education (Aikenhead \& Ogawa, 2007; Allen \& Crawley, 1998; Barnhardt \& Kawagley, 2005), environmental science (Berkes, Colding, \& Folke, 2000; Cajete, 2000; Kawagley \& Barnhardt, 1998; Tsuji \& Ho, 2002), and sustainability literature (Battiste, 2002;

Chambers \& Gillepsie, 2000; Murry, James, \& Drown, 2013). When considering Native and scientific communities working together or Native students undergoing science education, these authors suggest that conflict and confusion will arise (whether inter- or intra-individual) due to differing value orientations or worldviews between the two cultures.

For example, Aikenhead and Ogawa (2007) articulated that in a Western or EuroAmerican sense, scientific knowledge is founded upon beliefs that nature is knowable 
through data, universal truth is possible, parts can be summed to understand the whole, data comes through measurement, and reality and perceptions of reality are distinct. As a consequence, methods of discovering knowledge can be applied to any and all experiential domains, follow a linear progression, and include reductionism, quantification, replication, and generalizability within the contexts of funded pathways and societal interests.

In a Native or traditional sense, or what Aikenhead and Ogawa (2007) refer to as "Indigenous Ways of Living with Nature," indigenous knowledge is based on beliefs that nature is knowable through relationship, truth is place-based and changing, the whole is different than its parts, data comes from experience and reflection, and our perceptions are part of reality. Consequently, methods for discovering knowledge are based on firsthand experience, changing iteratively to adapt to new environments, circularity in time and being, holistic and inter-generational observation, and a tolerance for mystery or multidimensional intersecting influences. It is argued that culturally responsive approaches should provide an avenue to work with, rather than against, such differences (Allen \& Crawley, 1998).

Learning styles. Learning style has been defined as "the cognitive, affective, and physiological factors that serve as relatively stable indicators of how learners perceive, interact with, and respond to the learning environment (Swanson, 1995; Keefe, 1979). The initial idea came out the cognitive psychology work of Roger Sperry, who identified that right and left brain hemispheres served different cognitive functions (Sperry, 1961). Right brain functions were said to involve visual, creative, inductive, and holistic 
processing while left brain functions are dedicated to verbal, structured, deductive, and sequential processing. Eventually the concept was used to explain sub-group differences in educational outcomes and make suggestions for tailoring instruction for minority/atrisk youth who were presumably more "right-brained" (e.g., Ross, 1982; Walker, 1995).

As the concept evolved models and taxonomies of learning styles proliferated (Curry, 1987; Felder \& Spurlin, 2005; Rayner \& Riding, 1997; Riding \& Cheema, 1991); some being less extensive (e.g., Kolb, 1981) than others (e.g., Dunn \& Dunn, 1992). Researchers have debated the evidence and utility of building instructional methods around particular learning styles because of the significant situational and within-group variation, and have since argued for simply more varied learning environments (Dunn, Beaudy, \& Klavas, 2002; Pashler, McDaniel, Rohrer, \& Bjork, 2008). Nevertheless, there has been a fair amount of research on Native Americans learning styles that are worth considering with regard to the value of culturally responsive approaches (Berry, 1966, 1969, 1971; More, 1984; 1987; 1990; Kleinfeld \& Nelson,1988).

Hilberg and Tharp (2002) reviewed the empirical literature to conclude that higher percentages of Native American learners are global (holistic) thinkers, visually oriented, reflective, and collaborative compared to their White peers who fall on opposite ends of those continuums. Global, or holistic, thinkers prefer to consider pieces of information simultaneously through first hearing broad overviews and context to conceptualize a problem. This is contrasted with sequential thinkers who prefer more linear, piecemeal sets of information. Visually-oriented thinking as a term is selfexplanatory in that it describes a benefit from visual aids to process information (versus 
text only). Reflective thinkers tend to consider an issue before acting (versus trial-anderror), and collaborators are more likely to prefer group work, shared goals and responsibilities, and shared rewards (versus competitive orientations; see also a review by Pewewardy, 2002). The incorporation of lesson plans that provide overviews and metaphors to give learning purpose, group work and visual materials, and sufficient time to process before acting should all be included in a culturally responsive design for Native Americans.

Etiquette (Communication norms). The potential for miscommunication between Native students and Anglo teachers was identified in the dissertation work of Susan Urmston Phillips (1972) on the Warm Spring reservation in Central Oregon. Over the course of her research, she documented how communication in the home and in cultural life was very different than in the classroom (Phillips, 1976). Native communication tended to involve less talking, slower and softer speech, more listening, showing attention inadvertently (less gazing), requiring attention subtly (i.e., less eye contact), surround a physical activity, and use less body language. In addition, there are rules of etiquette for youth when speaking to each other that differs when speaking to adults. That style of communication was contrasted with that found in the classroom, which is much more verbose, boisterous, competitive, sedentary, and demanding of attention. Similar results were found amongst the Apache in the Southwest (Ingalls \& Hammond, 2007). Pueblo author and scholar Greg Cajete (1999) argues that communication and behavioral norms like the ones documented by Phillips are often wrongly labeled by teachers as defiance, laziness, or a lack of engagement or ability. Culturally responsive designs should help to 
address these types of miscommunications and the consequences to students or trainees that they happen to. In an employee training situation, cross-cultural miscommunication could threaten the employee's opportunities at work, especially if satisfactory completion of a training program depends on trainer appraisal.

Two words of caution should be mentioned with regard to the literature above: firstly, describing noticeable trends is altogether different than group uniformity. As it should have been clear from the section above on Native diversity, when it comes to individual students, they may or may not ascribe to the categories and thought-processes projected onto them as there is considerable within-group variation and contextual considerations (McCarty, Lynch, Wallace, \& Benally, 1991). Secondly, all epistemologies, learning and communication styles are experienced along continuums across all humanity. Despite the fact that particular cultures may emphasize particular values or behave in certain manners does not imply that they are exclusive to one or another group.

Race relations. Before I end this section on student need for culturally responsive designs, it is important to mention the issue of racism. The effect of racism might be broken down into contemporary and historical consequences. Contemporary racism represents students' experiences with discrimination within their lifetimes. In a Native sample in the upper Midwest, Whitbeck, Hoyt, McMorris, Chen, and Stubben (2001) found that across 10 forms of discrimination, $32 \%$ of Native students on average experienced one form or another. The most frequent was teachers being surprised at a student's academic success (54\%), someone yelling a racial slur at them (51\%), and 
being insulted for being American Indian (49\%). The experience of discrimination has been found to predict decreases in variables that are important to performance, such as self-esteem and social functioning in Native students (Galliher, Jones, \& Dahl, 2011) along with substance use (Whitbeck, Hoyt, McMorris, Chen, \& Stubben, 2001).

More informative is the suggested effect of historical racism. In a critique of CRE, educational anthropologist John U. Ogbu (1992a; 1992b; 1995; Ogbu \& Simons, 1998) argued that "Multicultural education may indeed improve school learning for some minority children. However, for several reasons it is not an adequate strategy to enhance the academic performance of those minorities who have traditionally not done well in school" (1992b, p. 6). He named the de-emphasis of students' personal responsibility, the inadequacy of classroom research to infer norms and differences of a cultural community, and, most importantly, the variations in minority achievement that suggest underlying cultural factors beyond similarity and/or dissimilarity.

Ogbu (1992; Ogbu \& Simons, 1998) delineates that minorities can be distinguished by their voluntary or involuntary status. While both have culture and language differences, voluntary minorities typically overcome related difficulties and after a year or two begin to attain high marks (e.g., West Indians, Koreans, and Burakmin in the US). Voluntary minorities also report positive framings of learning English and American culture, beliefs in the value of education, educated family members, and social pressures and supports to do well in school. Involuntary minorities, however, have persistently poor outcomes in school (e.g., Blacks and Natives here in the US; Koreans and Burakmin in Japan; West Indians in Britain). To account for this disparity Ogbu 
(1992b) further distinguishes cultural characteristics as primary (pre-contact) and secondary (post-contact), and argues how the relationship between the two cultures was initiated matters for how secondary cultural characteristics develop. He describes the process of cultural inversion, where involuntary minorities "do not seem to be able or willing to separate attitudes and behaviors that result in academic success... because such attitudes and behaviors are considered 'White"' (Ogbu, 1992b, p. 10; 1995). Success, defined in this way as assimilation, adds risk to achievement that may cause internal conflict and isolation from otherwise socially supportive networks. This risk is compounded with acknowledgement that the reward of equal pay and opportunity for minorities may not be available despite an education (Ogbu, 1992b; Ross et al., 2012). Wood and Clay (1996) used Ogbu's theory to explain differences they found between White and Native American students. They hypothesized that the perceived opportunities of school for one's career would translate into academic success. They found that while self-esteem and socialization into the dominant culture predicted good grades for White and Native students, the value of school to future jobs and school attachment only predicted grades for White students. The authors argued that this was the case because Native students did not believe the relationship between school and work was true for them. This might also help to explain an early study by Kerbo (1980) that found identification with Whites and integration with Whites (i.e., number of White friends) predicted Native success in college. It is possible that Native students face additional barriers to education in that their self-concept excludes them from academic success. This self-conception is both reactionary of real conditions as well as protective 
of ethnically-tied resources. In a case study of three Natives in Ivy League institutions, Brayboy (2004) reported how students work to keep their Native-ness invisible out of the interest of maintaining cultural integrity while excelling in school.

The take-away message from this section on race relations is that Native learners (e.g., students or trainees) may be resistant to achieve due to the beliefs that high performance would equate to "selling out" and that achievement would not result in the promised benefits anyways (i.e., expectancy). Depending on the organizational context, similar beliefs may impact the effectiveness of training. For CRE approaches to address this power-based sense of exclusion, the original formula of Ladson-Billings (1994) that included a goal of developing students' critical conscientiousness may be necessary to instill a sense of efficacy and purpose to participation.

\section{Summary}

In this section I defined important concepts and argued their applicability to the training context. First I defined culture and considered its potential effect on training lesson receptivity. Secondly I defined culturally responsive education and suggested that similarly culturally inclusive designs might assist the learning process. Third, I reviewed its significance for Native Americans as a cultural group with a unique political and cultural history. The review detailed the potential of culturally responsive designs to 1) accommodate diversity (e.g., ranging from broad to specific cultural groups), 2) inform needs assessments (e.g., for cultural identifiers), 3) fulfill old promises (e.g., especially for government agencies), 4) distance training platforms from learning environments with negative connotations, 5) respect tribal sovereignty, 6) close the achievement gap, 7) tie 
training into cultural value orientations, 8) utilize pre-existing learning styles, 9) help avoid cross-cultural offenses, and 10) help address trust issues stemming from experiences with racism. However, although culturally responsive designs have been called for by Native educators and students (Barnhardt, 1994; Lee \& Quijada-Cerecer, 2010), before these multiplicative benefits can be realized the initial claim of CRE must be evaluated: Does CRE increase learning and motivation?

In the next section, I review the evidence for CRE with Native American learners, describe the theory of CRE effectiveness, and acknowledge of the current gaps in the empirical research. Finally, I combine the evidence for CRE with theory from the training literature to develop research questions and hypotheses regarding CRE in the training context that I plan to test. 


\section{Evidence, Theories, \& Hypotheses}

In the second section I reviewed the expansive literature on effective training design and noted that, despite its relevance, training people of different cultural groups is a currently understudied area. Afterwards I defined CRE as a pedagogical approach that may contribute to research on culture in the training context and described its significance for Native American organizations, communities, students, and employees. In this section, I review the evidence that CRE increases performance and/or influences important learning mechanisms for Native Americans and explain how the research supports/fails to support the theoretical models of its effectiveness. Afterwards I draw upon theories of training and development from the industrial-organizational literature to make predictions in the form of hypotheses.

\section{Early Research - 1971-2003}

Research on how to improve the education of Native Americans blossomed in the 1970 's following supportive legislation, ${ }^{6}$ continued into the 1980 's, and grew substantially in the 1990's. In 1998, President Clinton's Executive Order on AI/AN education (\#13096) requested an evaluation of the effective strategies and promising approaches to closing the achievement gap, especially those that involved native languages and cultures (Clinton, 1998, p. 42682). As part of that research agenda, over 100 articles, books, dissertations, and theses were exhaustively reviewed and summarized by Tlinget scholar William G. Demmert Jr. for Washington D.C.'s Office of Educational

\footnotetext{
${ }^{6}$ Indian Education Act of 1972; Self-Determination and Educational Assistance Act of 1975.
} 
Research and Improvement (Demmer, 2001) and Portland's Northwest Regional Education Lab (Demmert \& Towner, 2003). Because Demmert's extensive reports have become reference guides for culturally responsive education research with indigenous students, I chose the date of the later report to distinguish the early period from the current period in this young line of research. The later report was chosen as it was largely based on the articles from 2001 review, with some minor updates.

Not surprisingly, Demmert (2001) found that improving Native education entailed interventions at multiple locations and points of an individual's life. He organized research on Native success in education into six themes: early childhood environment and experiences; Native language and cultural programs in schools; teachers, instruction, and curriculum; community and parental influences on academic performance; student characteristics; and factors leading to success in college. While a full synopsis of the report is beyond the scope of my review, of particular importance are his conclusions about the evidence for culturally responsive instruction on learner outcomes.

Demmert (2001) reported, "a series of studies in the past 30 years collectively provides strong evidence that native language and cultural programs - and student identification with such programs - are associated with improved academic performance, decreased dropout rates, improved school attendance rates, decreased clinical symptoms, and improved personal behavior" (p. 17). Further, Demmert, McCardle, Mele-McCarthy, and Leos (2006) wrote that Demmert's 2001 report provided, "Information on teachers, instruction, and curriculum [that] tells us that teachers competent in their subject areas, 
given a variety of instructional approaches and a challenging, culturally-based curriculum, can motivate students to do well in school," (p. 94).

Notable examples from this report included language programs, culturally integrative programs (i.e., in content and design), and teaching/instruction studies. ${ }^{7}$ Stiles (1997) compared and contrasted four language programs with diverse indigenous groups; Cree, Hualapai (see also Watahomigie \& McCarty, 1994), Native Hawaiians, and the Maori (of New Zealand) and pointed out that while each program used different outcome criteria, each reported progress in one area or another. Benefits included increased graduation rates, decreased dropout rates, and strengthened pride in one's identity. Rudin (1989) similarly found improved native vocabulary and ethnic pride in Omaha children who participated in a language renewal program. Culturally integrative programs included a curriculum development project was associated with an increase in student reading and listening comprehension scores with Navajo students and an enduring sense of empowerment for Navajo teachers (Lipka \& McCarty, 1994). Another is the Alaska Native Knowledge Network's (ANKN, 1998) statewide program that included teacher training and parent and community involvement, but focused on increasing math achievement and reducing dropout. Schools affiliated with the program showed consistent decreases in bottom quartile math scores for $8^{\text {th }}$ and $11^{\text {th }}$ graders, unlike schools that were not part of the program. Teacher-instruction studies found that teachers did well when they took a teacher-as-learner position (Cleary \& Peacock, 1998) and that

\footnotetext{
${ }^{7}$ Demmert (2001) uses this taxonomy to provide examples of “culturally-based education," a variant of culturally responsive education.
} 
student participation and engagement can be increased by alternative conversational styles (Tharp \& Yamauchi, 1994), flexible classroom organization (Soldier, 1988), use of cooperative learning, computers, and cultural knowledge (Bennett, 1987; Swisher, 1990).

From the initial report, it would seem that culturally responsive approaches are strongly supported by the research. Such conclusions must be tempered however as Demmert (2001) admittedly did not assess the quality of the research for his exhaustive review (p. iv). Perhaps more informative is the subsequent report by Demmert and Towner (2003) that revisited this literature with a more critical eye toward methodology. Studies were screened by two criteria: They had to use a) CRE interventions and b) experimental or quasi-experimental designs. Demmert and Towner (2003) delineated that to be considered a CRE design six components needed to be present: 1) recognition and use of Native languages, 2) pedagogy that stresses traditional cultural characteristics, 3) pedagogy that uses teaching strategies that are congruent with traditional culture, 4) curriculum based on traditional culture and recognizes the importance of Native spirituality, 5) strong Native community participation, and 6) knowledge and use of the socio-political norms of the community (p. 9-10). Experimental designs were those characterized by questions of causation, included random assignment, and control groups. Quasi-experimental designs were evaluations that were, for whatever reason, unable to randomize assignment into treatment or control groups. Quasi-experimental conclusions were stronger if they had preliminary data to compare or statistically control for naturally occurring group differences. 
Of the over 100 studies reviewed by Demmert (2001), Demmert and Towner (2003) found that, "nearly all of the research consisted of qualitative case studies and simple descriptions. Of all the studies reviewed, only six studies targeting culturally based education could be considered experimental or quasi-experimental, and only one speaks directly to the culturally based education/academic achievement link ...

Obviously, there is a strong need to design and implement research studies that will yield valid and reliable information" (p. 7).

The most well-established study was conducted on the Hawaiian reading program Kamehameha Early Education Program, or KEEP (Tharp, 1982). The program was evaluated through three experiments of increasing generalizability. The first was a pilot study that compared classes who had received varying levels of the KEEP program; one class who had never received the intervention, three classes that received it all three years, one only two years, and one only one year. The second experiment tried the program in public schools where students who volunteered to participate were compared to students whose parents did not enroll them. Both intervention and control groups were considered "high risk." The third experiment implemented the program in three locations in Hawaii but enrollment was based off of random assignment. In each study students in the program outperformed their comparison group. Tharp (1982) makes a point that development of this program was very intentional about integrating aspects of traditional Polynesian culture into the pedagogy of the comprehension-based intervention. This was particularly in regard to the 'ohana, or kin-based group, that still practices traditional cooperating and instructional styles, like share-functioning (i.e., shared responsibility 
with unspecific roles), shared-resources, sibling-caretaking, and peer-orientation.

Unfortunately, the effect of the culturally responsive element was confounded with other aspects of the intervention and attempts to import the success of this program has been found to require much institutional support (Greymorning, 1997; Lipka \& McCarty, 1994)

Less intensive studies that contained CRE instruction and used experimental or quasi-experimental designs included an unpublished manuscript by Lipka and Adams (2002) on culturally-based geometry instruction and Kratochwill, McDonald, BearTibbitts, and Levin's (2001) evaluation of a parental involvement program. Lipka and Adams (2002) randomly assigned teachers to teach one or another type of instruction in both urban and rural schools, each with classrooms that served as controls. Percent correct of students' pre- and post-tests was compared with series of $t$-tests that showed culturally-based instruction outdid control groups. Kratochwill et al. (2001) identified and matched 3 pairs of schools that were randomly selected to implement the Families And Schools Together (FAST) program. FAST is designed to improve social behavior and school retention through parent participation, although academic achievement is also measured. The program failed to have a significant effect on academic achievement but did improve students' social functioning. The other studies that Demmert and Towner (2003) recognized for their experimental designs either did not evaluate the effect of any culturally responsive component of the program or did not measure achievement or both. Omizo and Omizo (1989) and Omizo, Omizo and Kitaoka (1998) tested the effect of art 
activities on the self-esteem of Hawaiian children and Clark (1996) tested a computer assisted instructional program with First Nations (Indigenous Canadians) students.

To compensate for the scant selection of experimental studies, Demmert and Towner's (2003) included a supplemental section on eight comparative studies. Comparative studies describe control groups to juxtapose the effects of their treatment groups, however group formation does not involve random assignment or matched samples, and groups tended to exist outside of the research context. These ex post facto designs are acknowledged to have weak internal validity, but are included to provide breath to the conclusions where experimental research is silent. Six of these were language or bilingual programs and two were focused on math achievement. Bilingual programs either produced better English speakers (Cottrell, 1971; Franks, 1988; Murtagh, 1982) or increased general academic performance (Bacon, Kidd, \& Seaberg, 1982; Rosier \& Holm, 1980; Wright, Taylor, \& Macarthur, 2000). Math program evaluations included the statewide project in Alaska described above (ANKN, 1998) and Brenner's (1998) mathematics contribution to Hawaii's KEEP program; both showed improvements to student math scores.

Following their review, Demmert and Towner (2003) observed that as the criteria used in research were allowed to be less stringent the conceptual focus was more closely honed in on question of interest. They concluded, "What is needed, of course, is scientifically rigorous research that is on target regarding culturally based education as an educational treatment or program" (p. 31). They end this early period of research on CRE with four basic research prescriptions to improve research in this area: 
1) Carefully define culturally-based education interventions,

2) Target student learning as an outcome,

3) Include estimates of effect size, and

4) Design research with an adequate comparative base.

\section{Current Research 2003-2014}

Over ten years have gone by since Demmert and Towner's (2003)

recommendations. The research since that time has been fruitful, but still not heeding to the standards of empirical rigor requested in their report. I searched a series of electronic databases (PsyINFO, EBSCOhost, and Google Scholar) with the terms "Native American," "American Indian," "Alaskan Native," "Indigenous;" "culturally-responsive," “-congruent," or “-tailored," “-sensitive;" "education," "curriculum," “schooling," "teaching," or "pedagogy." I recovered thirty-nine articles that devoted sections on culturally responsive education for Native Americans, but only six that studied the culturally responsive approaches systematically. Twenty-three of the articles were process papers, commentaries, literature reviews, and theoretical works related to Native education that did not involve any primary data collection (e.g., Aguilera, Lipka, Demmert, \& Tippeconnic, 2007; Barnhardt \& Kawagley, 2005; Brayboy \& Castagno, 2008, 2009; Pewewardy, 2003; Tharp et al., 2007). Of the sixteen remaining articles that did include research (fourteen qualitative, two quantitative) ten did not study culturally responsive education directly. For instance, Balter and Grossman (2009) interviewed educators about the impact of No Child Left Behind (NCLB). Educators mentioned how 
NCLB required teachers to teach toward the test and compromise the use of teaching methods related to their culture.

Qualitative studies that addressed cultural responsiveness specifically included two case studies and two interviews, one of each with a focus on science education. Science education has been argued to be of great importance to the competitiveness of our nation's workforce in general (Augustine, 2005) and any attempts to achieve environmental sustainability worldwide (World's Scientific Academies, 2000). However, science education has been argued to be particularly relevant to Native communities for three unique reasons: 1) they have lesser access to resources (i.e., barriers to development) that require science-based solutions (e.g., 10x more likely not to have electricity, Energy Information Administration, 2000; more likely to be exposed to environmental hazards, Lewis, 1995; James et al., 2008), 2) they are generally placebased people, with economic, health, and cultural investments in local geography (Semken, 2005), and 3) indigenous ecological knowledge has and should continue to contribute to mainstream science (Cajete, 2000; Barnhardt \& Kawageley, 2005). In an article largely dedicated to describing the conceptual elements of his research program on geoscience education in general (e.g., Riggs, 1998; Riggs, Robbins, \& Darner, 2007), Riggs (2004) describes a case study with Southern California’s Indigenous Earth Sciences Project. Although no outcomes were reported, he concludes that the program was successful in attracting and retaining students, to which he attributed the combination of field-based learning, place and problem-based structures, and incorporation of traditional ecological knowledge (or TEK) to be the cause. Mack et 
al. (2012) interviewed 30 educators in informal science education programs that focus on engaging Native youth in science while incorporating indigenous culture. Their interviews were read and analyzed by a Consensus Advisory Committee formed of twelve subject matter experts in the field of indigenous education. The results mimic past commentaries and theoretical works for culturally responsive education to include recommendations such as "Use the community as an integral resource in developing curriculum and instruction," "Use Native language to facilitate instruction," and "Match the values of the program to the values of the people" (p.67). The conclusion from these qualitative studies is that program designers perceive culturally responsive geoscience to work well with their indigenous students (see also Semken, 2005) and that the community is asking for such programs.

The other two qualitative studies focused more generally on culturally responsive classrooms. Hickling-Hudson and Alquist (2003) compared case studies of four schools in the U.S. and Australia, two that incorporated aboriginal culture and two that did not. They noted that culturally responsive schools validated student identity and teachers earned trust with their families, and at least in one school students scored high on standardized tests. These were contrasted with the latter two schools, of which they described an overwhelming Anglo-centricity in texts and instructional style that perpetuated the perspective that, "it is white men who made history; discovered other lands; shaped the histories of science, the arts, and humanities; and made the important contributions to the world" (p. 81). No comparable metrics on student tests were provided from these schools. Rogers and Jaime (2010) interviewed five Native community 
members for practical guidance for emerging culturally responsive non-Native educators. Three themes were distilled from the interviews: 1) learn from the community, 2) transform thinking through discomfort (e.g., cross-cultural within-person conflict management), and 3) gain awareness of positive values (i.e., reframing for empowerment). These qualitative studies provide evidence that schools that are culturally responsive differ from schools that are not, and that there are practices advocated by the community to help non-Native teachers make the transition. Unfortunately, these studies do not help to answer the question of whether or not culturally responsive designs are responsible for increases in learning and performance or how that happens.

Of the two quantitative studies I identified, the first did not study CRE's effect or its mechanisms and the second found negative results. Roehrig, Dubosarsky, Mason, Carlson, and Murphy (2011) evaluated the Ojibewe teacher training program Ah Neen Dush for its ability to impart inquiry-based and culturally relevant skills in science education. After two years of the program, teachers showed a statistically significant increase in emotional support variables (i.e., positive climate and regard for student perspective), classroom organization (i.e., productivity, learning formats, and overall), and instructional support (i.e., quality of feedback and overall). No results were reported regarding student performance nor were the degrees to which inquiry-based verses culturally responsiveness drove the effect. López, Heilig, and Schram (2013) analyzed data from the National Assessment of Educational Progress combined with the National Indian Education Study's data on Native's school experiences. In a hierarchical linear model controlling for school membership and a range of confounds at individual and 
school levels (e.g., absenteeism; teacher certifications), López et al. (2013) found a negative relationship with an item on teacher's inclusivity of Native culture and NAEP scores in grades 4 and 8 . Although this one item doesn't actually measure CRE and could refer to even stigmatizing lessons that include Native culture, the negative relationship does demonstrate that cultural integration, perhaps improperly implemented, is not necessarily a positive thing.

In 2006, Native educators produced a "research blueprint" on what information was currently needed to advance Native student success in academia (Demmert et al., 2006). One of the five major items was, "examining the effectiveness of culture-based education in comparison to existing instruction" (p. 104). Unfortunately, my review of the literature did not reveal any experimental studies that provided evidence specific to CRE's effectiveness. One important trend is the sheer number of arguments and rhetoric concerning CRE and its perceived association with self-determination and cross-cultural engagement. Although it is technically beyond the scope of my review, the same trend is occurring internationally.

For example, in my literature search I also found fifteen articles calling for culturally responsive education in Canada and Australia. Of the eight Canadian articles, only three included original research and none provide experimental evidence of CRE (Agbo, 2004; Santamaria, 2009; Sterenberg, 2013). Abgo (2004) found that First Nations community members, teachers, and band council members wanted CRE, Santamaria (2009) identified schools with improving scores and showed how they integrated CRE with differentiated instruction (see Tomlinson, 2000), and Sterenberg (2013) reported a 
case study of a teacher who attempted to integrate math lessons framed in both Western scientific and Indigenous frameworks but failed to be able to do so.

Likewise in Australia, while seven articles called for CRE for aboriginals, only two collected data on for an empirical project. In a mixed method study, Turner, Richards, and Sanders (2007) found that parental education that used culturally tailored materials and group-based designs were effective in reducing neglect and child symptoms compared to a control, although no non-culturally responsive segment was offered. In Santoro, Reid, Crawford, and Simpson, (2011), interviews with Indigenous instructors found that integrating TEK, understanding students lives outside of class, and building relationships with aided in their effectiveness as teachers. Although there is a movement nationally and internationally by educators, community members, and politicians, and there is abundance of well-reasoned arguments for culturally approaches, unfortunately the data is no available in the field of education back up the claims. My project therefore contributes to the overall culturally responsive framework across educational platforms, and although my focus will pertain to culturally responsive forms of adult training by implication my results will fill a much need gap in the education literature as well.

\section{Theoretical Framework}

Theories of why CRE is expected to be effective can be derived from the CRE or the training literature. Neither field is known for a preoccupation with theory, however both offer explanatory frameworks with which to construct models and make hypotheses. In the CRE literature, the three most often cited theories of CRE are Compatibility Theory (Tharp \& Gillmore, 1989), Cultural-Historical-Activation-Theory (CHAT) and 
Cognitive Theory (Tharp, Estrada, Dalton, \& Yamauchi, 2000). Demmert and Towner (2003) remind us that most studies only subscribe to a particular theory by implication, and that an attempt to sort studies by theoretical position proved to be a useless venture due to the small number of studies that used theory to contextualize their approach explicitly (p. 9).

Compatibility Theory asserts that problems in learning and performance can stem from differences in behavioral and cognitive expectations between home and school environments. According to Tharp (1989), school and home culture differ across four sets of variables: social-organizational, socio-linguistic, cognitive, and motivational. As a justification for CRE, Compatibility Theory would suggest that CRE reduces these differences. Cognitive theory posits that learning takes place most substantially when new information is processed through associations with prior knowledge and schemas. The greater the number of associations, the more likely information will be remembered and understood. Within this framework, CRE would increase the number of opportunities for students to connect new information with past knowledge and experiences. Whereas cognitive theory applies to any previous knowledge base or conceptual framing, Tharp and Gillmore (1989) pulled upon Vgotsky’s Cultural-Historical-Activity Theory to describe a broader form of association that takes place, when new information is introduced within the larger context of a child's socialization. They argue that new information's relation to tangible and intangible symbols of culture, history, and community activity are the most likely to result in retention. 
In the training literature, as discussed in the section on training, Baldwin and Ford's (1988) theory of training transfer has remained the most popular explanatory and predictive framework. They posited that training design, trainee characteristics, and the post-training environment (e.g., workplace) individually and collectively support or hinder learning during training and maintenance of learned content afterwards (i.e., transfer):

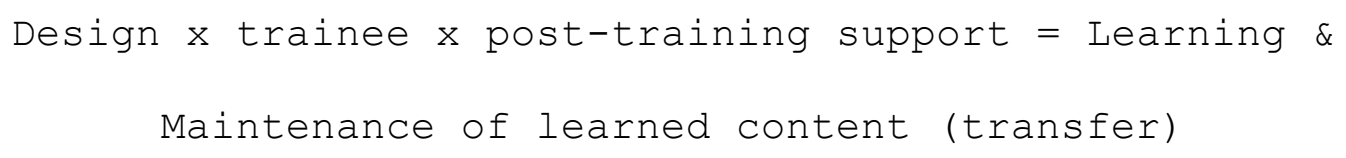

Within this framework, the CRE approach can be viewed as set of training design decisions (i.e., responsiveness) that accommodate culturally-tied trainee characteristics related to learning and performance. The emphasis on design and trainee characteristics suggests that other theories and models of training are relevant to CRE approaches.

Design. With regard to design characteristics, models of training in evaluation delineate where CRE impacts the trainee. Bushnell (1990) outlined IBM's Input-ProcessOutput (IPO) and Outcomes training evaluation model to explicate the links between different elements and stages of the training process (see Figure 1). In his rubric, training is the combination of 1) inputs: trainee qualifications, instructor abilities, materials, funding, facilities, 2) process: the planning, design, development, and delivery of the program, 3) outputs: trainee reactions, KSA's gained, enhanced performance, and 4) outcomes: more output, more profit, better product/customer satisfaction. By definition, CRE informs particular inputs (e.g., instructor abilities, materials) and the general process. The IPO model stipulates that for a culturally responsive training to be valid, it would need to demonstrate the ability to enhance outputs (e.g., trainee reactions, KSA's) 
and outcomes more than a training without the cultural responsiveness. Applying Kirkpatrick (1976) and Alliger et al.'s (1997) division of training outcomes, CRE could also differentially produce affective outcomes of liking and utility judgments depending on the purpose or success of the training.

Figure 1. IPO model of training evaluation. Reprinted from "Input, Process, Output: A Model for Evaluating Training," by D.S. Bushnell, 1990, Training and Development Journal, 44, p.41. Copyright 2003 by EBSCO publishing.

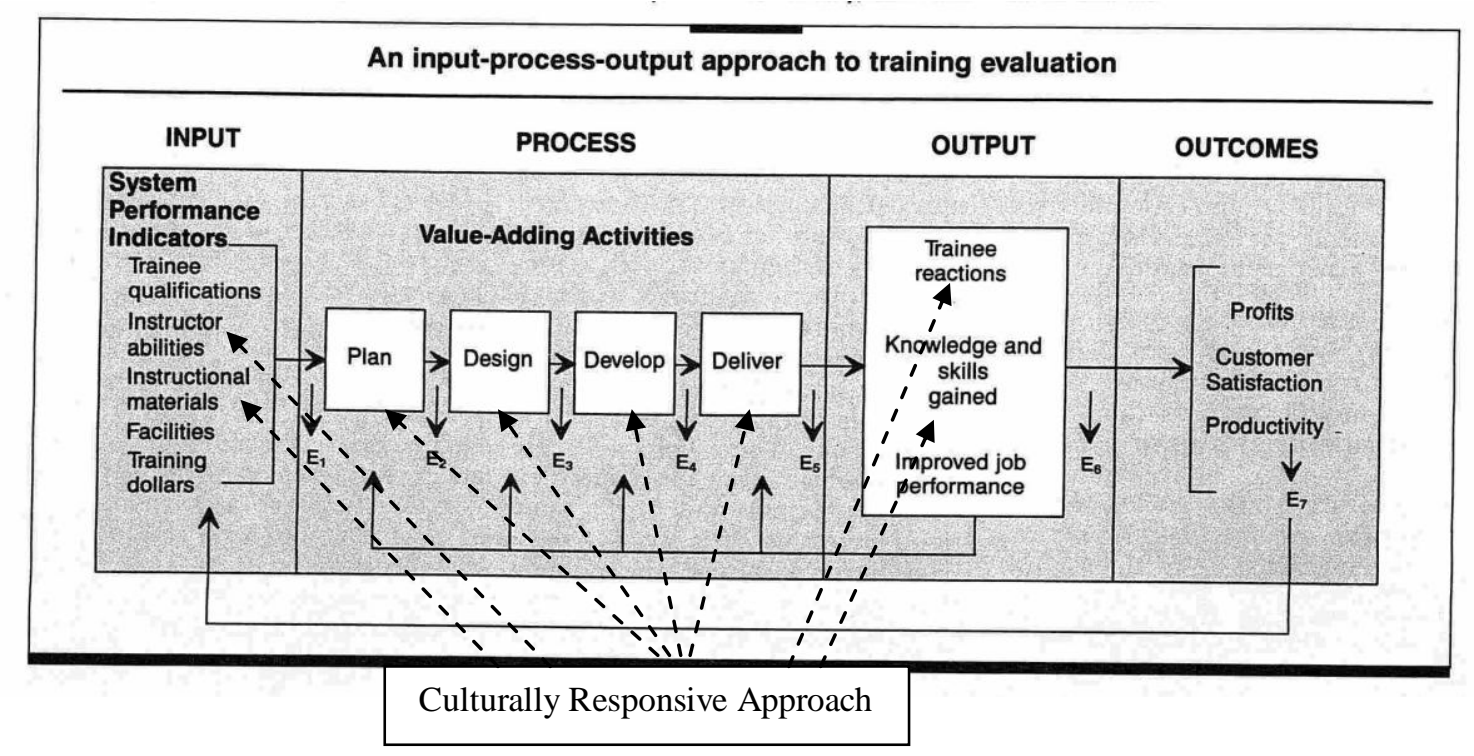

Trainee characteristics. The crux of CRE is its potential to capitalize on trainee characteristics that are shared by a cultural group. According to Baldwin and Ford (1988), trainee characteristics include ability, personality, and motivation. Since their foundational text this list has been expanded to include a range of variables (Colquitt et al., 2000). Of interest are those which would be affected by culturally responsive instruction. In other words, if CRE does in fact increase the output of trainees from a particular cultural group, how does it do it? What are the mechanisms that increase performance for trainees of this particular group? 
According to Compatibility Theory and CHAT, CRE should work by making the learning process and content more familiar and relevant to trainees' lives (Lemke, 2001; Roth \& Lee, 2007). Increased familiarity and relevance of the curriculum are not likely to be associated with more or less stable constructs, e.g., ability or personality, however each is related to elements of motivation that have been demonstrated to influence training effectiveness. For example, more experience with an environment (i.e., familiarity) is associated with higher self-efficacy and the relevance of a learning experience to actual consequences increases the perceived usefulness (i.e., instrumentality) and perceived importance (i.e., valence). Expectancy (i.e., self-efficacy) has been shown to interact with instrumentality and valence to motivate behavior in training settings (Howard, 1989; Vroom, 1964) and self-efficacy and valence (characterized as goal commitment) were found to moderate the effect of goal setting on effort and performance (Locke \& Latham, 1990; 2002). Colquitt et al. (2000) showed in their meta-analysis that self-efficacy and valence predicted motivation to learn, which in turn predicted several training outputs and outcomes.

Another trainee characteristic of importance is identification with the topic matter (or program or field), particularly for involuntary minorities. According to Ogbu's (1992) theoretical work, involuntary minorities internalize a certain amount of resistance to mainstream standards of achievement. This is partly reactionary as persistently inequitable access to resources weakens the belief in self-efficacy and instrumentality. Identification with an occupation or field or program symbolizes a sense of belonging and commitment to an intellectual, school, or workplace community. 
In summary, culturally responsive approaches are expected to improve outputs (e.g., learning, reactions) through aligning inputs and processes with trainee characteristics to create more optimal contexts for learning (Baldwin \& Ford, 1988; Bushnell, 1990). Theoretically speaking, this alignment improves the process by: 1) reducing demands on social interaction, emotion, and cognition caused by cultural conflict (Lemke, 2001; Tharp, 1989), 2) heightening relevance through framing training objectives with collectively-valued meanings and consequences (Gay, 2000; LadsonBillings, 1994; Tharp, Estrada, Dalton, \& Yamauchi, 2000), and 3) increasing comprehension through the provision of more associative networks (Tharp, Estrada, Dalton, \& Yamauchi, 2000). If CRE functions as it is theorized to do, then CRE should improve learning performance, self-efficacy, motivation, goal-commitment, and identification of the trainees over non-culturally responsive approaches for designated cultural audiences.

\section{The Current Study}

My review demonstrated the need for research on training and trainee culture (Brown \& Sitzmann, 2011) and more scientifically rigorous research on CRE (Demmert \& Towner, 2003). Case studies and qualitative research from schools and educators support the utility of CRE (Hickling-Hudson \& Alquist, 2005; Mack et al., 2012; Riggs, 2004) and some theoretical development has outlined its mechanisms (Gay, 2000; Ladson-Billings, 1995; Tharp, 1989; Tharp et al., 2000). The literature revealed however that the basic tenant, that CRE improves learning outcomes, still needs to be vetted through the experimental process (Demmert \& Towner, 2003). Therefore, I hypothesize 
that trainees who are exposed to a CRTI will acquire more knowledge and skills during training than trainees exposed to a non-CRTI. In addition, CRE scholars have made the case that one of the ways CRE facilitates a productive learning environment is by increasing motivation to learn, self-efficacy, identification with the material, and commitment to learning objectives (Demmert, 2001, Gay, 2000, Tharp \& Yamauchi, 1994). While this belief is consistent with research in T\&D that has demonstrated the mediating effect of affective motivators on learning during training (e.g., motivation to learn and self-efficacy; Colquitt, LePine, \& Noe, 2000), it has not been experimentally validated in a CRE context (Demmer \& Towner, 2003). I hypothesize that trainees who are exposed to a CRTI will have higher ratings on affective motivators (i.e., motivation to learn, self-efficacy, identification with the material, and goal-commitment) compared to trainees who received a non-CRTI. Further, I hypothesize that the effect of CRE on knowledge and skill acquisition will be mediated by increases in affective motivators. The full model is displayed in Figure 2.

I tested these hypotheses in the context of a data-use training program built for OIEA. Preliminary training needs assessment interviews and surveys at two OIEA board meetings and the OIEA 2013 conference guided the content development. Fifteen OIEA members took the survey, although six either did not use research for their work or did not provide information or ratings regarding their use of data. Of the nine members who listed their uses of data and rated the frequency and importance of their data-use, members reported they used data sometimes to a lot $(M=2.46$ on a scale from $0-3)$ and that their use of data was very important $(M=2.97$ on a scale from $0-3)$. Members listed 
an average of 4.33 reasons for using data and the top three member-requested training objectives were how to read/understand what data says, how to question the results, and how to use it practically and apply it to their work. More specifically, a) how to use and understand existing data for problem-framing in grant applications and b) how to create data to provide evidence of currently-funded programs (i.e., evaluation).

Figure 2. Proposed model of culturally responsive pre-training intervention's effect on knowledge, skill, and affective outcomes, with affective outcomes mediating the effect of the intervention on knowledge and skills.

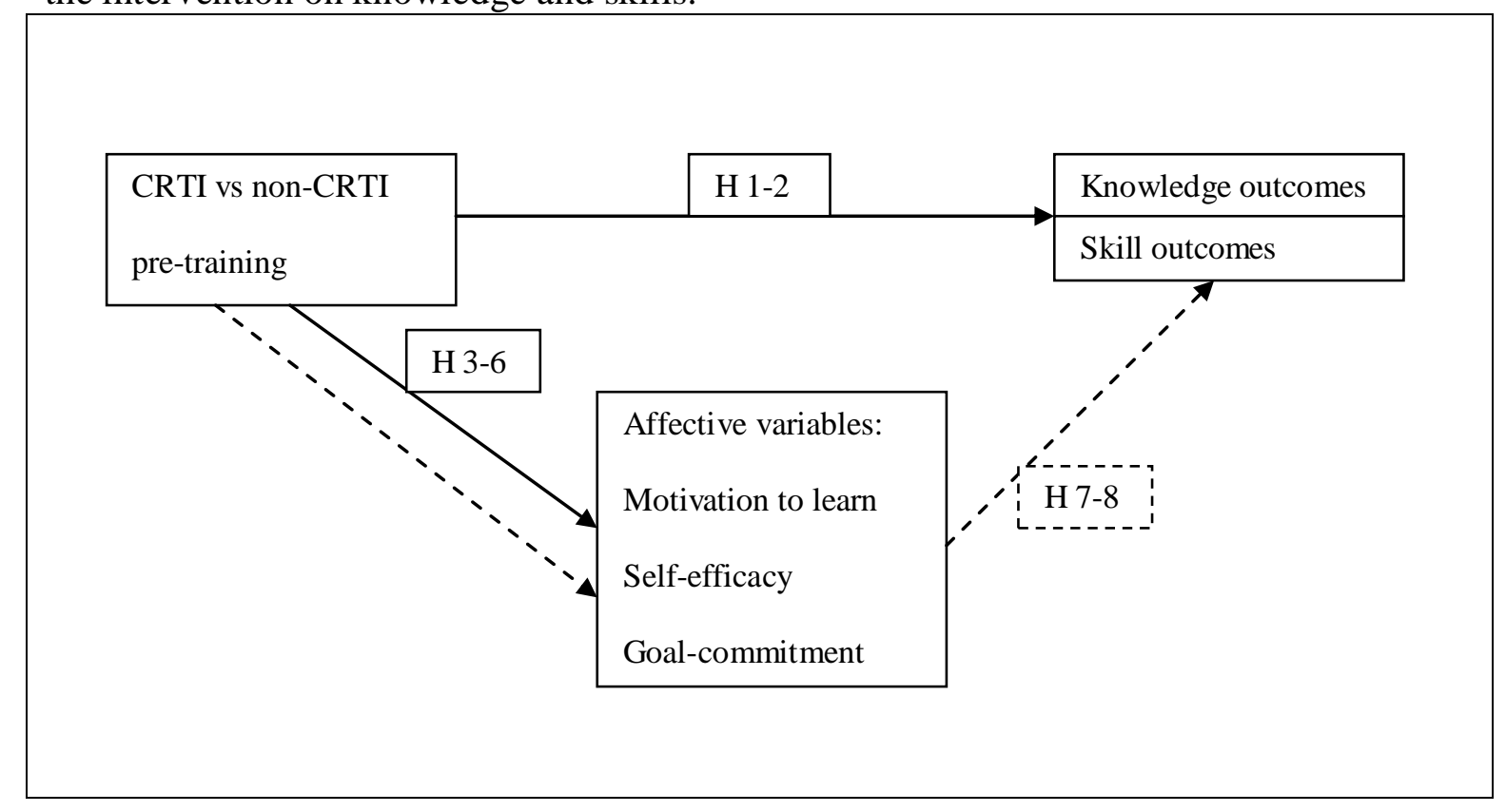

To the extent possible, the components outlined in the sections on CRE were included in the CRTI tested here. Table 1 outlines the various taxonomies of CRE by author. In line with the requests by Demmert and Towner (2003) to specify what is meant by CRE when it is tested, I placed an " $\mathrm{X}$ " under CRE components that were integrated into my CRTI manipulation. As Table 1 shows, my CRTI did not include every possible expression of CRE outlined in the literature and more closely followed prescriptions from 
multicultural education in general. Concessions had to be made given that a) the CRTI was delivered via video rather than through interactions with the trainees, which made b) the incorporation of more extensive CRE frameworks inaccessible (Barnhardt \& Kawagaley, 2005; Castagno \& Brayboy, 2008), c) CRE formulations were primarily focused on child and youth education, and e) implementation was usually described within the school context rather than a one-day occupational training, which made certain recommendations difficult if not out of place (e.g., family events). In the current study the question of dosage is not being addressed. Incremental increases in the prescribed methods of cultural responsiveness were not tested. 
Table 1. Components of CRE included in CRTI

\begin{tabular}{|c|c|c|c|c|}
\hline \multicolumn{5}{|c|}{$\begin{array}{l}\text { Components of Culturally Responsive Education by Authors } \\
\qquad \text { An "X" below the component signifies its inclusion in this training prime }\end{array}$} \\
\hline \multirow[b]{2}{*}{ Theme } & \multicolumn{4}{|c|}{ Author/Focus } \\
\hline & $\begin{array}{c}\text { Banks \& Banks (2009) } \\
\text { CRE in general }\end{array}$ & $\begin{array}{c}\text { Demmert \& Towner } \\
\text { (2003) CRE for Natives }\end{array}$ & $\begin{array}{c}\text { Gay (2000) } \\
\text { CRE in general }\end{array}$ & $\begin{array}{c}\text { Ladson-Billings (1994) } \\
\text { CRE in general }\end{array}$ \\
\hline \multirow[t]{2}{*}{ Integration } & Content integration & $\begin{array}{l}\text { Recognition \& use of } \\
\text { language }\end{array}$ & knowledge base & \\
\hline & & & $\mathrm{X}$ & \\
\hline \multirow[t]{2}{*}{ Curriculum } & Knowledge construction & $\begin{array}{l}\text { Curriculum based on } \\
\text { traditional culture and } \\
\text { recognizes the importance } \\
\text { of spirituality }\end{array}$ & $\begin{array}{l}\text { Designing Culturally } \\
\text { Responsive Curriculum }\end{array}$ & $\begin{array}{l}\text { Focuses on academic } \\
\text { success }\end{array}$ \\
\hline & & & & $\mathrm{X}$ \\
\hline \multirow[t]{2}{*}{ Interactions } & Equity pedagogy & $\begin{array}{l}\text { Pedagogy that emphasizes } \\
\text { traditional cultural } \\
\text { characteristics }\end{array}$ & $\begin{array}{l}\text { Cross-cultural } \\
\text { communication }\end{array}$ & $\begin{array}{l}\text { Develops cultural } \\
\text { competence }\end{array}$ \\
\hline & & & & $\mathrm{X}$ \\
\hline \multirow[t]{2}{*}{ Self-awareness } & $\begin{array}{l}\text { Safe, non-prejudicial } \\
\text { learning environment }\end{array}$ & $\begin{array}{l}\text { Knowledge and use of } \\
\text { socio-political norms }\end{array}$ & & $\begin{array}{l}\text { Develops critical } \\
\text { consciousness }\end{array}$ \\
\hline & $\mathrm{X}$ & $\mathrm{X}$ & & $\mathrm{X}$ \\
\hline \multirow[t]{2}{*}{ Inclusion } & $\begin{array}{l}\text { Empowering school } \\
\text { culture/structure }\end{array}$ & $\begin{array}{l}\text { Native community } \\
\text { participation }\end{array}$ & $\begin{array}{l}\text { Demonstrating cultural } \\
\text { caring/building learning } \\
\text { community }\end{array}$ & \\
\hline & & $\mathrm{X}$ & $\mathrm{X}$ & \\
\hline Congruity & & $\begin{array}{l}\text { Pedagogy that uses } \\
\text { teaching strategies } \\
\text { congruent with traditional } \\
\text { culture }\end{array}$ & $\begin{array}{l}\text { Congruity between } \\
\text { home/school culture }\end{array}$ & \\
\hline & & & & \\
\hline
\end{tabular}

Note 1. Dimensions of culturally responsive education are grouped by theme across rows. It should be noted that this thematic organization is not definitive due to overlapping and dissimilar nuances between authors' definitions. This chart should be considered a rough approximation and not a claim of essential agreement between scholars. 


\section{Method}

\section{Partnering Organization}

This study was initiated and made possible through collaboration with the Oregon Indian Education Association (OIEA). OIEA is a state-based non-profit organization that works to increase Native student success in K-12 and higher education. Their activities include: 1) maintaining a network of professionals who are actively involved in Native education, 2) disseminating information about important educational programs, legal cases, cultural events, scholarship and internship opportunities, and updates regarding Native studies and student resources at schools and universities, 3) hosting two annual conferences, one for educators/researchers and one for youth, and 4) holding board meetings that pass resolutions and discuss activities mentioned above. OIEA and its constituents work with local tribal populations (e.g., Siletz, Warm Springs, Grande Ronde) or with tribally diverse Urban Native populations (e.g., Portland). For this project, OIEA aided in the advertisement, recruitment, organization, and design of the training as well as with community outreach for volunteers.

\section{Sample}

The current sample is comprised of 67 constituents of the Oregon Indian Education Association (OIEA) who attended a day-long data-use training. Members worked in various aspects of Indian education in Oregon and Washington (see Table 2). Trainees were either Native American (80.6\%) or White (14.9\%) for the most part (two Asians and one Other), however $28.4 \%$ reported being Native American and White ( $n=$

19). Trainee age ranged from $20-67$ years $(M=43.95)$, tended to be female $(n=53$, 
$79.1 \%$ ), educated (17.9\% had an associate degree, $20.9 \%$ a bachelor's, and $43.3 \%$ had a master's degree or above), and report a median annual household income $(\underline{m}=\$ 50-60 \mathrm{k})$ on par with the national median $(\underline{m}=\$ 51,939$, DeNavas-Wilt \& Proctor, 2014).

Table 2. Sample characteristics by occupation and location by data collection time point.

Sample characteristics by occupation and location by data collection time point

\begin{tabular}{|c|c|c|c|c|}
\hline Occupation & & & $n$ & $\%$ \\
\hline Title VII Indian education specialist & & & 20 & 30 \\
\hline Director or manager of Indian education programs & & & 13 & 19 \\
\hline Program coordinator in Indian education & & & 13 & 19 \\
\hline Program assistant & & & 6 & 9 \\
\hline Intern of Indian education program & & & 5 & 7 \\
\hline Community advocate & & & 4 & 6 \\
\hline Teachers & & & 3 & 4 \\
\hline Academic (faculty or student) & & & 2 & 3 \\
\hline Tribal representative & & & 1 & 1 \\
\hline Unemployed & & & 1 & 1 \\
\hline Location & & & & \\
\hline & $n$ & $\%$ & $n$ & $\%$ \\
\hline Tacoma, WA & 18 & 27 & 15 & 26 \\
\hline Portland, OR & 15 & 22 & 7 & 24 \\
\hline Klamath Falls, OR & 12 & 18 & 11 & 19 \\
\hline The Confederated Tribes of Warm Springs Reservation & 8 & 12 & 8 & 14 \\
\hline Springfield, OR & 5 & 7 & 1 & 2 \\
\hline The Confederated Tribes of Umatilla Indian Reservation & 5 & 7 & 5 & 9 \\
\hline $\begin{array}{l}\text { The Confederated Tribes of Coos Bay, Lower Umpqua, and Siuslaw } \\
\text { Indian }\end{array}$ & 4 & 6 & 4 & 7 \\
\hline Total & 67 & 100 & 58 & 100 \\
\hline
\end{tabular}

These sample characteristics indicate that training attendees represent Native

American professionals and are not be confused with Native Americans in general, who are younger on average $(M=32.1)$, have less, formal education $(9.2 \%$ with a bachelor's, $4.7 \%$ with more than a bachelor's), and earn a lower median annual hou sehold income than the national rate ( $\underline{m}=\$ 36,641$; American Community Survey, 2013).

According to an a priori power analysis using the moderate to large effect sizes from Tharp's (1982) KEEP program evaluation ${ }^{8}\left(\oplus^{2}=.077-.145\right)$, an alpha of .05 , and

\footnotetext{
${ }^{8}$ This was the only quantitative, quasi-experimental study that evaluated CRE on learning outcomes to date.
} 
power at .80, I estimated a necessary sample size of 59-133 participants using a betweensubjects regression with six predictors to represent my exogenous variables. In the attempt to incorporate the repeated measures nature design I am using for this project, I also ran a power analysis with an effect size comparable to omega-squared for a withinbetween subjects MANOVA; alpha of .05, power at .80, and a medium-to-large effect size (Cohen's f: between .25-.40). The estimated sample ranged from 52-128. It should be noted that the sample estimate here is based on power calculations that do not account for the additional internal validity added by my manipulation and location-based random assignment.

\section{Procedure}

Training invitations and flyers were sent out via OIEA's listserve as an "OIEAdata-use training for Native educators." Occasionally flyers and invitations were circulated through within-organization listserves. The introductory letter contained a description of the general purpose of the training, who should attend, and how to sign up. In four of the six locations local coordinators handled all of the recruitment, room rental, and registration, otherwise I coordinated rooms, recruitment, and registration online. Participants received no incentive other than the learning experience and breakfast and lunch during the data-usage session. In some cases, attendees' employers paid for their time as the training was considered work-related.

A training session lasted all day (9am - 4:30pm), began with introductions and a humble breakfast. Participants then viewed a pre-training intervention video, took the initial survey, listened to slide-based lecture, ate lunch, participated in group activities, 
and ended the day by completing the exit survey. A peripheral goal of this study is to evaluate the overall effectiveness of this training to facilitate learning and increase positive attitudes toward research pre- to post-test. The central goal however is to test the impact of a culturally responsive intervention on learning and motivational factors.

\section{Data-use Training}

Data-use training had 3 objectives: to inform, to equip, and to inspire. In other words, it focused on providing information, building skills, and influencing affective variables related to comprehending data and conducting research. Data-use and research methods were taught in the context of a) problem framing and b) program evaluation for grant applications and grant accountability reporting. End goals were stated on an introductory slide as:

1. Should be familiar with fundamental concepts \& terms

2. Should be able to select metrics for evaluation

3. Should be able to interpret findings from descriptive data Content was divided into three parts: 1) Data for problem framing and program evaluation, 2) data collection and measurement, and 3) calculating and presenting results. Part 1 described large scale survey data on American Indian student outcomes, the use of percentages, means, and standard deviations to demonstrate need, and the types and designs of program evaluation (e.g., experimental/non-experimental studies). Participants were asked to imagine a program they work on or want to work on and identify key variables. Part 2 discussed sampling issues and definitions, operationalization (e.g., constructs versus variables), scaling decisions, reliability, and the validities (i.e., 
internal/external, convergent/discriminate). Participants were asked to create four questions that can be answered on a scaled response, one for each category: demographic, belief, attitude, and behavior. Participants then move around the class and collect data from fellow trainees. Part 3 contains a short description of different presentation options for data (e.g., crosstabs, bar charts, scatterplots) by data type (e.g., nominal by nominal v. ordinal by ordinal). Afterwards, trainees are asked to calculate their data with the statistic of their choice (e.g., sum, mean) and draw a graph that shows the relationships between their variables (or lack thereof) and present it back to the group.

To the extent possible, empirically-based recommendations were implemented in the delivery of this training (e.g., role modeling, error management, and feedback). The trainer participated in the activities with participants so as to be available for questions and lead by example (role-modeling), it is explicitly mentioned and written on the “activity" slides "Remember that it is okay to make mistakes," and I offer feedback on every presentation.

Focal outcome variables (described further below) included questions on datausage knowledge (e.g., the difference between averages and percentages; samples versus the population) and data-usage skills (e.g., quality of interpretation of a histogram/pie chart; operationalizing a variable). Data collection followed a design standard for a program evaluation with pre- and post-training surveys to assess learning as a result of program participation (Rossi, Lipsey, \& Freeman, 2003). 


\section{Manipulation: Culturally-responsive pre-training intervention}

My culturally responsive intervention was an 11- to 12-minute video delivered prior to a training's pre-test survey and lecture content (Prime A, see Table 2). The video was a series of edited and integrated interviews with three Native researchers (two male, one female; two $\mathrm{PhDs}$, one $\mathrm{PhD}$ candidate) who conduct research for the benefit of Native American communities. The interviews asked about what motivates them to do their work, how they stay committed to their goals, whether research was difficult in their opinion, what has helped them to gain confidence and identify with research, and how their work benefits the communities they work for. The video was targeted toward Native American pan-Indian culture, rather than specific to a tribe. Pan-Indian culture, sometimes referred to as Native Nationhood, relates to the shared cultural characteristics of tribal Nations in North America, including Alaska and Canada. Central elements to pan-Indian culture include the experience of and resistance to colonialization, indigeneity (importance of place to identity and symbolism), emphasis on spirituality, collectivist values, and tribal identification. The introduction incorporated flute music by local Kiowa/Comanche artist and Pacific Northwest tribal art.

The culturally responsive pre-training intervention was randomly assigned to location order and kept hidden from the instructor to the extent possible. If the culturally responsive intervention was not assigned, those participants received a content-matched video but from non-Native (i.e., White) researchers (two male, one female, one $\mathrm{PhD}$; two doctoral candidates). This alternative video (Prime B) was created as a control for the 
effect of receiving a motivational video, albeit minus the direct references to Native community work and culture (see Table 2).

Table 3. Training component delivery sequencing.

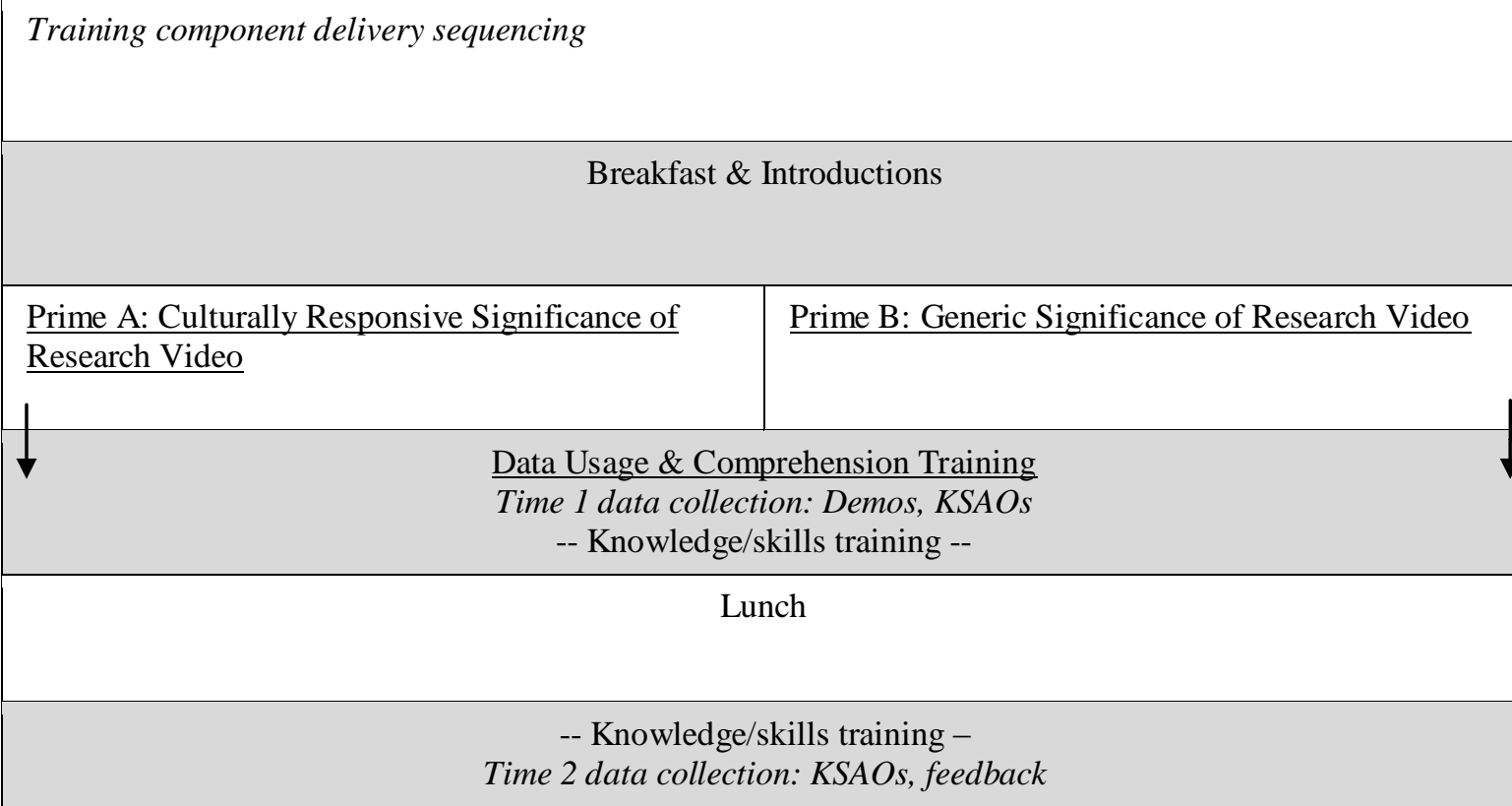

\section{Measures}

Prior to the training evaluation survey, participants watched one of two randomly assigned videos to compare the effect of culturally responsive content. A short survey was given to participants to fill out while they watched the video as a manipulation check. After that survey was completed, the participants completed the official training evaluation survey, which included items that were both unique to each test occasion as well as repeated between time 1 and time 2 .

Manipulation check. To ensure that trainees paid attention to the video prime, participants were asked to fill out a video evaluation. Ratings were asked for the video's audio and video quality, pace, clarity, relevance, and cultural appropriateness, with an 
open-ended question on the trainee's subjective take-away message. This evaluation was for an independent variable manipulation check and not intended for analysis.

Integrity of the training implementation was coded with regard to the number of activities a session successfully made it through (out of three). Various unexpected delays, some training-supportive some not, made it impossible to complete the activities as intended. An interval scale from 1-3 was created to control for the effect of incomplete hands-on examples.

A sequence variable was created that essentially numbered the training by the order they were given. This rank ordinal variable allows me to evaluate the effect of my improvement as a trainer and increased familiarity with the material over time. With 7 training, the sequence variable is on a scale from 1-7.

Pre- and post-training surveys. Surveys were handed out before and after the data-usage component of the training. Time 1 (pre-training) and Time 2 (post-training) surveys both contained unique items as well as repeated measures (see Table 4). Pretraining-only questions included demographics, covariates (i.e., research experience and cultural identity), and motivation to learn. Repeated measures included knowledge and skill assessments and three affective motivators. Post-training only items included reactions, feedback, and future interests.

Demographics. Demographic information were collected to assess the sample population served by OIEA's training program and to control individual differences. Information included age, gender, job title, type of employment, ethnicities, tribal affiliation, education, and income. Demographic information was described in the sample 
Table 4. Name and number of items for measured constructs by survey occasion.

Name and number of items for measured constructs by survey occasion

Time 1 (Pre-training)

Occupational type

Occupational title

Age

Gender

Average annual household income

Ethnicity

Education level

Cultural identity

Research experience

Research knowledge

Research skills

Motivation to learn from training

Research self-efficacy

Research goal-commitment

Identification with research
Time 2 (Post-training)

Research knowledge*

Research skills*

Research self-efficacy*† Research goal-commitment* $*$ Identification with research*†

Utility of training Suggestions for improvement Evaluation cultural appropriateness Interest in other training modules

Note: Constructs measured pre-training (time 1) are listed in the left column while constructs measured post-training (time 2) are listed in the right column. Repeated measures appear in both pre- and posttraining columns and are marked with an asterisk. The number of items per construct is listed in the center column $(i=$ number of items). Change in constructs marked $\dagger$ were not tested in this study.

section. For supplementary analyses ethnicity was recoded to reflect sub-groups that were

salient to my sample composition (see Table 5).

Table 5. Ethnicity recode, sample size, and percentage of total sample.

Ethnicity recode, sample size, and percentage of total sample

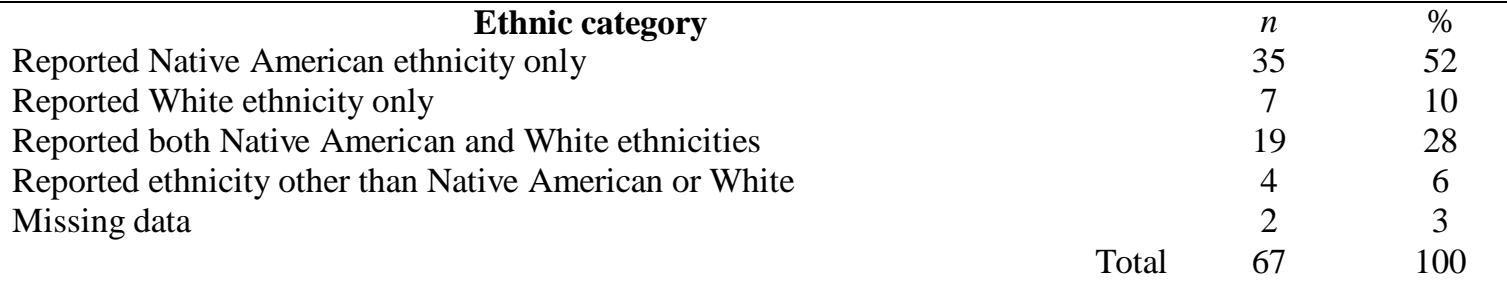

Knowledge. Knowledge was assessed through 12 items on concepts and terms that were taught during the training and basic to research (see Table 6 and Appendix A).

Three items were from the National Science Foundation's Science Indicators survey on 
understanding the scientific process and probability (Miller, 1998; 2004) and 9 were adapted from curriculum from an upper division psychological research methods course at Portland State University (Cozby \& Bates, 2012). Most of the items $(i=10)$ were dichotomously scored as correct or incorrect except for an open-ended Science Indicators item that requested an explanation for the participant's previous answer on whether or not to use a control group in the provided scenario. That item was scored on a scale from 0-3, where $0=$ no answer, $1=$ any attempt, $2=$ mentioned systematic process, $3=$ mentioned control group (Miller, 1998). Answers were summed for a total knowledge score.

Table 6. Scoring rubric for knowledge and skill assessments. Scoring rubric

\begin{tabular}{lc}
\hline \multicolumn{1}{c}{ Question topic } & Scale \\
\hline Knowledge & $0-1$ \\
Medicinal drug research scenario - use control & \\
group or not & $0-3$ \\
Explanation of answer & $0-1$ \\
Probability & $0-1$ \\
Population versus sample & $0-1$ \\
Variable types (e.g., continuous) & $0-1$ \\
Reliability versus validity & $0-1$ \\
Definition of confounded variable & $0-1$ \\
Experimental versus correlational research & $0-1$ \\
Variable versus construct & $0-1$ \\
Definition of independent variable & $0-1$ \\
Definition of dependent variable & $0-1$ \\
Basic versus applied research & 14 \\
& \\
Skill Total points possible & \\
Identify independent variables & $0-2$ \\
Identity dependent variable & $0-1$ \\
Interpret bar chart & $0-3$ \\
Calculate mean from data & $0-1$ \\
Create graph from data using mean & $0-3$ \\
\end{tabular}

The conventional cut-off for a "good" test item's item-total correlation is .25, with a minimally acceptable cut-off at .15 (Varma, 2008). Higher item-total correlations indicate that a right answer on an individual item is related to a higher total score more 
than we would expect from random chance. In other words, that correct answers to a particular item is related to overall knowledge rather than guessing. The desired median item-difficulty (percent correct) for a four-option multiple choice response format is around $75 \%$ (Lord, 1952) which indicates that the test is not too easy or too difficult (i.e., moderate difficulty). Knowledge items performed well overall in both times 1 and 2 (see Table 7).

Table 7. Knowledge and skill assessment performance statistics.

Test performance statistics

$\begin{array}{lcc}\text { Knowledge } & \text { Pre-training } & \text { Post-training } \\ \text { Average percent correct (range) } & & 75 \%(45 \%-97 \%) \\ \text { Median percent correct } & 65 \%(13 \%-94 \%) & 75 \% \\ \text { Average item-total correlation } & .31(.09-.53) & .28(-.03-.49) \\ \text { Skill } & & \\ \text { Average percent correct (range) } & 67 \%(42 \%-91 \%) & 73 \%(55 \%-86 \%) \\ \begin{array}{l}\text { Median percent correct } \\ \text { Average item-total correlation (range) }\end{array} & 66 \% & 72 \% \\ \end{array}$

Three knowledge items did not perform well in Time 1 . The most aberrant was a question on probability $\left(r_{\mathrm{i}-\mathrm{i}}=.09\right)$, but this is likely due to the high percent correct $(94 \%)$ which left little variance in the item. It was included in the composite. The two other items that failed to meet the "good" item cut-off improved in the post-test scores and were therefore kept in the composite as an indicator of change. In Time 2 two items had very poor inter-item correlations, but again the percent correct was so high (.88 and .97 respectively) that this is likely due to a lack of variance in the items. In addition, these items still technically passed minimally acceptable guidelines $\left(r_{\mathrm{i}-\mathrm{i}}\right.$ range $\left.=.14-.21\right)$.

Skills. Skills were assessed with 5 items related directly to comprehending and interpreting information from the Oregon Department of Education (ODE). Graphs were 
taken from ODE's Statewide Report Card (Saxton, 2014) which is available online and contains information relevant to Native educators in the state, such as tables, charts, graphs, and descriptions of achievement scores, disciplinary actions, and dropout rates by year, grade, gender, and ethnic groups. Trainees were asked to both interpret a graph (i.e., on dropout rates for Oregon high school students 2012-2013) and create graph with an interpretation (i.e., based off a mean disciplinary action rates for student in 2013-2014 by ethnicity; see Table 6 for scoring rubric). Skill items were summed for a total score and measured both pre- and post-training (times 1 and 2).

Pre- and post-training skill items performed well overall (see Table 7). In the post-training assessment, inter-item correlations increased and the item difficulty decreased as expected post-training. Interestingly, there was a drop in both attempts (91 to $86 \%$ ) and in detail (60 to $43 \%$ ) from pre-test to post-test answers on the graph interpretation. This was possibly due to the shortage of time to complete surveys at the end of the day in addition to participant fatigue post-training.

Affective variables. I used four affective variables measured at Time 1: trainingrelated motivation to learn, self-efficacy, goal-commitment, and identification with research. All affective scales were measured using a 5-point Likert-type scale from 0-4, zero representing strong disagreement and 4 representing strong agreement. I evaluated scale structure using principal components analysis (PCA) on the sample correlation matrix with oblique rotation and confirmatory factor analysis (CFA). PCA is useful when the goal is to describe the qualities of scales within a sample instead of generalizing components to a larger population (Tabachnick \& Fidell, 2007), oblique rotation was 
used to allow components to correlate. Variable decisions regarding composites or factor scoring were informed by PCA results and created based on fit indices produced in the CFA's. Scale reliability was evaluated using Cronbach's alpha as a measure of internal consistency (see Table 8).

Pre-training motivation to learn. Training-related motivation to learn was measured using an adapted version of Zaniboni, Fraccaroli, Truxillo, Bertolino, and Bauer's (2011) expectancy-based 9-item scale. This scale measures motivation to learn through the components outlined in Vroom's (1964) expectancy theory, that is, valence, instrumentality, and expectancy. Valence is defined as one's affective orientation (e.g., attractiveness, desirability, or importance) toward the immediate benefits of training (e.g. learning, improved skills). Instrumentality is the belief that training results in job-related benefits. Expectancy is the confidence that the participant can learn the training material if they are involved. Sample items include, "I feel that it is important to take part in this training in order to strengthen my research skills," "I believe the training activity is useful for workers who occupy a job position similar to mine," and "If I am involved in this training, I am confident I can improve my ability to do research.” The three-factor model and has shown to be both reliability and validity in Zaniboni et al.'s (2011) cross-cultural sample (Cronbach's alpha, $\alpha=.86, .78$, and .89 respectively).

$\mathrm{Hu} \&$ Bentler (1999) recommend CFI and TLI statistics above .95, an RMSEA below at least .08, although .05 is preferred, and an SRMR of less than .09. A higher order factor for motivation to learn with three subscales (i.e., valence, instrumentality, expectancy; $i=3$ each sub-scale), as defined in Zaniboni et al. (2011), fit the data well 
$(\mathrm{CFI}=.99, \mathrm{TLI}=.99, \mathrm{RMSEA}=.04, \mathrm{SRMR}=.03)$. I therefore created mean composite scores per sub-scale as in the theory-based validation study. Each sub-scale had excellent internal consistency: valence $(\alpha=.95)$, instrumentality $(\alpha=.80)$, and expectancy $(\alpha=$ $.93)$.

Pre-training self-efficacy. For research self-efficacy, three components of Bieschke, Bishop, \& Garcia’s (1996) 4-component Research Self-efficacy scale were borrowed from according to items' similarity to training' objectives (total $=16$ items). I adapted Bieschke et al.'s questions for Conceptualization, Implementation, and Presenting Results since they were related to the training content. Questions refer to the level of confidence that a participant has on a list research-related objectives. An example of conceptualization is "Generate researchable questions"; implementation, "Choose measures of dependent and independent variables;" and presenting results, "Orally present results to your research group."

A CFA testing a higher order factor with three subscales as defined in Bieschke et al. (2006; conceptualization, implementation, presentation) produced poor fit statistics $(\mathrm{CFI}=.83, \mathrm{TFI}=.80, \mathrm{RMSEA}=.16, \mathrm{SRMR}=.084) . \mathrm{I}$ suspected that this was due to the substantial number of cross-loading of items on multiple components and two rogue items identified in the PCA as driving much of the second factor; the only two items related to using computers (i.e., to analyze data and generate graphics). Dropping cross loading and computer-efficacy items resulted in a well-fitting research self-efficacy model $(\mathrm{CFI}=.985, \mathrm{TFI}=.977, \mathrm{RMSEA}=.068, \mathrm{SRMR}=.043)$, wherein a higher order factor for research self-efficacy was defined by the three subscales used by Bieschke et al.. 
Table 8. Factor structure and fit indices by scale.

Factor structure and fit indices by scale

\begin{tabular}{|c|c|c|c|c|}
\hline $\begin{array}{l}\text { Construct } \\
\text { Subscales }\end{array}$ & $i$ & Initial factor structure & Revised factor structure & $\alpha$ \\
\hline \multirow[t]{2}{*}{ Motivation to learn } & 9 & CFA single-factor model & $\begin{array}{l}\text { CFA higher-order factor } \\
\text { model* }\end{array}$ & \\
\hline & & $\begin{array}{l}\text { CFI }=.87, \text { TLI }=.82, \text { RMSEA }=.20 \text {, } \\
\text { SRMR }=.065\end{array}$ & $\begin{array}{l}\text { CFI }=.99, \text { TLI }=.99 \\
\text { RMSEA }=.04, \text { SRMR }=.03\end{array}$ & .55 \\
\hline Valence & 3 & & & .95 \\
\hline Instrumentality & 3 & & & .80 \\
\hline Expectancy & 3 & & & .93 \\
\hline \multicolumn{2}{|l|}{ Chi-Square } & & $\chi_{\text {diff }}^{2}=72.86, p<.001$ & \\
\hline Self-efficacy & 16 & $\begin{array}{l}\text { CFA higher-order factor model } \\
\text { with subscales }\end{array}$ & $\begin{array}{l}\text { CFA higher-order factor model } \\
\text { with cross-loading items } \\
\text { removed* }\end{array}$ & .96 \\
\hline $\begin{array}{l}\text { Self-efficacy without } \\
\text { cross-loading items }\end{array}$ & 9 & $\begin{array}{l}\text { CFI }=.83, \text { TFI }=.80, \text { RMSEA }= \\
.16, \text { SRMR }=.084\end{array}$ & $\begin{array}{l}\text { CFI }=.985, \text { TFI }=.977, \\
\text { RMSEA }=.068, \text { SRMR }=.043\end{array}$ & .93 \\
\hline Conceptualization & 3 & & & .90 \\
\hline Implementation & 3 & & & .83 \\
\hline Presentation & 3 & & & .94 \\
\hline \multicolumn{2}{|l|}{ Chi-Square } & & $\chi_{\text {diff }}^{2}=1741, p<.001$ & \\
\hline \multirow[t]{2}{*}{ Goal-commitment } & 5 & CFA single-factor model & $\begin{array}{l}\text { CFA higher-order factor model } \\
\text { with second lower-order latent } \\
\text { variable for reverse code items* }\end{array}$ & .72 \\
\hline & & $\begin{array}{l}\text { CFI }=.90, \text { TFI }=.80, \text { RMSEA }=.16 \text {, } \\
\text { SRMR=.09 }\end{array}$ & $\begin{array}{l}\text { CFI }=.997, \text { TFI }=.969 \\
\text { RMSEA }=.063, \text { SRMR }=.019, \\
\chi_{2}^{2}=12.25, p=.02\end{array}$ & \\
\hline Chi-Square & & & $\chi_{\text {diff }}^{2}=12.25, p=.02$ & \\
\hline \multirow{2}{*}{$\begin{array}{l}\text { Identification with } \\
\text { research }\end{array}$} & 9 & CFA single-factor model* & & .76 \\
\hline & & $\begin{array}{l}\text { CFI=.95, TFI=.93, } \\
\text { RMSEA=0.06, SRMR=.07, } \\
\text { ACI=1225). }\end{array}$ & & \\
\hline \multirow{3}{*}{$\begin{array}{l}\text { Research experience } \\
\text { Experience with } \\
\text { Research } \\
\text { Occupational } \\
\text { supportfor } \\
\text { research }\end{array}$} & 4 & CFA single-factor model & CFA two-factor model* & .66 \\
\hline & 2 & $\begin{array}{l}\text { CFI }=.92, \text { TFI }=.76, \text { RMSEA }=.15 \text {, } \\
\text { SRMR }=.06\end{array}$ & $\begin{array}{l}\text { CFI }=1.0, \text { TFI }=1.10, \\
\text { RMSEA }=0.00, \text { SRMR }=.02\end{array}$ & .65 \\
\hline & 2 & & & .55 \\
\hline \multicolumn{2}{|l|}{ Chi-Square } & & $\chi_{\text {diff }}^{2}=4.65, p=.03$ & \\
\hline \multirow[t]{2}{*}{ Native identity } & 7 & CFA single-factor model & $\begin{array}{l}\text { CFA higher-order model with } \\
\text { second lower-order latent } \\
\text { variable for parental identities* }\end{array}$ & .86 \\
\hline & & $\begin{array}{l}\text { CFI }=.90, \text { TFI }=.90, \text { RMSEA }=.14 \text {, } \\
\text { SRMR }=.07\end{array}$ & $\begin{array}{l}\text { CFI }=.94, \text { TFI }=.90 \\
\text { RMSEA }=.13, \text { SRMR }=.06 \\
\chi_{\text {diff }}^{2}=4.62, p=.099\end{array}$ & \\
\hline \multirow[t]{2}{*}{ White identity } & 7 & CFA single-factor model & $\begin{array}{l}\text { CFA higher-order model with } \\
\text { second lower-order latent } \\
\text { variable for parental identities* }\end{array}$ & .89 \\
\hline & & $\begin{array}{l}\text { CFI }=.90, \text { TFI }=.86, \text { RMSEA }=.16 \text {, } \\
\text { SRMR }=.07\end{array}$ & $\begin{array}{l}\text { CFI }=.95, \mathrm{TFI}=.92, \\
\mathrm{RMSEA}=.12, \mathrm{SRMR}=.05 \\
\chi_{\text {diff }}^{2}=13.46, \mathrm{p}=.001\end{array}$ & \\
\hline
\end{tabular}

Note: $i=$ number of items; $\alpha=$ Cronbach's alpha across items, *model used to construct variable(s) 
This model was a statistically significant improvement over including the full item list $\left(\chi^{2 \mathrm{diff}}=226 ., 88, p<.001\right)$. I u sed the higher order factor score since I was interested in self-efficacy in research overall rather than its components. The items combined had excellent internal consistency $(\alpha=.93)$, similar to the total-item alpha reported for Bieschke et al.'s (1996) full 4-component Research Self-efficacy scale $(\alpha=.96)$. Subscales were internally consistent as well: conceptualization $(\alpha=.90)$, implementation $(\alpha=$ $.83)$, and presentation $(\alpha=.94)$.

Pre-training goal-commitment. Goal-commitment was measured using Klein, Wesson, Hollenbeck, Wright, \& DeShon's (2001) 5-item revision of the Hollenbeck, Williams, and Klein's (1989) 9-item goal-commitment scale. Hollenbeck et al. (1989) showed that the 5-item version showed significantly improved fit statistics over the longer model with only slightly lower internal agreement $(\alpha=.74)$. A sample item includes, "I am strongly committed to learning how to use research skills." A model where goal commitment was a higher order factor that was informed by positively framed goal-commitment item factor and a reverse-coded-items factor. This model showed the excellent fit $(\mathrm{CFI}=1.0, \mathrm{TFI}=1.07, \mathrm{RMSEA}=0.00, \mathrm{SRMR}=.024) . \mathrm{I}$ used the factor loading from the goal commitment component as the goal-commitment variable. The internal consistency (i.e., Cronbach's alpha) for goal-commitment's five items was $\alpha=.72$.

Identification with research. No scale for Identification with Research could be located, so a scale was constructed using 3 items from James and Cardador's (2007) Technology and Self sub-scale of their Cognitions and Beliefs about Technology and Science inventory and 6 items from Meyer, Allen, and Smith's (1993) the affective 
occupational commitment sub-scale, adapted for jobs in research. Example items include "People like me are good at research" and "I dislike the idea of being a researcher" (reverse-coded). The reliability of the James and Cardador's (2007) subscale was a little below convention at .64 in their validation study while Meyer et al.'s (1993) subscale had an internal agreement of .87. A CFA demonstrated that identification with research items all loaded on a single factor $(\mathrm{CFI}=.95, \mathrm{TFI}=.93, \mathrm{RMSEA}=0.06, \mathrm{SRMR}=.07)$ so $\mathrm{I}$ created a composite score across the 9 items. Internal consistency (i.e., Cronbach's alpha) for pre-test identification with research was $\alpha=.76$.

Covariates. Apart from demographics, pre-program survey controls included past research experience, occupational support for research, and cultural identity.

Research experience and occupational support. The extent that one uses research for their occupation was measured with four questions. Three were questions created for this survey (i.e., "How much experience have you had conducting research?", "How often do you have to use research for the work you do?", and "My role at work allows me to be involved in research") and the fourth came from the National Science Foundation (NSF) Science Indicators question bank (Miller, 1998; 2004). Created questions were rated on a 5-point Likert scale ranging from $0=$ none, never, strongly disagree to $4=$ a lot, all of the time or strongly agree. The NSF question (i.e., "Some things are studied scientifically; some things are studied other ways. Would you say you have (circle one)") was answered on a scale from 0-2, with zero representing "no understanding of its meaning," one for "a general sense of its meaning," and two for "a clear understanding." Due to the fact that the coding scheme was slightly different 
between the items created for this survey and the NSF question, answers were standardized so as to share the same scaling. Model fit significantly improved when research opportunities at work were loaded on one factor and experience and understanding were loaded on another $(\mathrm{CFI}=1.0, \mathrm{TFI}=1.10, \mathrm{RMSEA}=0.00, \mathrm{SRMR}=.02$, $\left.\mathrm{AIC}=622, \chi^{2 \mathrm{diff}}=4.65, p=.03\right)$ so I calculated factor scores separate for each. The internal consistency between work and personal experience (i.e., Cronbach's alpha) was $\alpha=.66$. Individually work opportunity items were more consistent $(\alpha=.65)$ than experience and understanding items $(\alpha=.55)$.

Cultural Identity. Cultural identity was measured to control for the effect that a Native American culture-focused program may depend on participants' identification with that in-group. Cultural identity was measured using Oetting and Beauvais' (19901991) Orthogonal cultural identification scale, where participants can identify with more than one cultural identity. The 7 item scale provides the first half of statements such as "Do you live or follow" or "Is your father successful in" with 6 response options for Native American, White, African-American, Latino, Asian, Other way of life. It was apparent that the ethnic composition of attendees was primarily either Native American, White, or both (see Table 5), therefore cultural identity scales for my participants were only calculated for Native American and White identity. CFA's run separately on Native identity $(\mathrm{CFI}=.94, \mathrm{TFI}=.90, \mathrm{RMSEA}=.13, \mathrm{SRMR}=.06)$ and White identity $(\mathrm{CFI}=.95$, TFI=.92, RMSEA=.12, SRMR=.05) both showed better fit when run as a higher-order factor with individually-related and family-related items loaded on separate lower-order 
factors. Higher-order-factor scores were used for Native and White identity. Internal consistency was acceptable for Native identity $(\alpha=.86)$ and White identity $(\alpha=.89)$.

\section{Analysis}

Preliminary descriptive analyses were run on raw composite scores to aid in interpretability, and evaluate central tendency (i.e., means), variability (i.e., standard deviations), normality, and kurtosis. Preliminary inferential statistics (i.e., correlations, one-way ANOVA's) were run to evaluate the quality of my manipulation and explore potentially confounding covariates that should be included in the larger model. Hypotheses were evaluated using a structural equation model (SEM) on mean-centered composite and factor scores (i.e., path analysis). The model is presented in Figure 3, where a CRTI predicts knowledge and skill acquisition above a topically-matched nonculturally responsive intervention. Individual differences in knowledge and skill were controlled for with pre-test data (not shown in the Figure 3). Direct and indirect effects were tested simultaneously, significance was interpreted at the conventional alpha $=.05$. 
Figure 3. Path analysis of CRTI on knowledge and skill acquisition, mediated by motivation to learn (MTL), self-efficacy, goal-commitment, and identification with research.

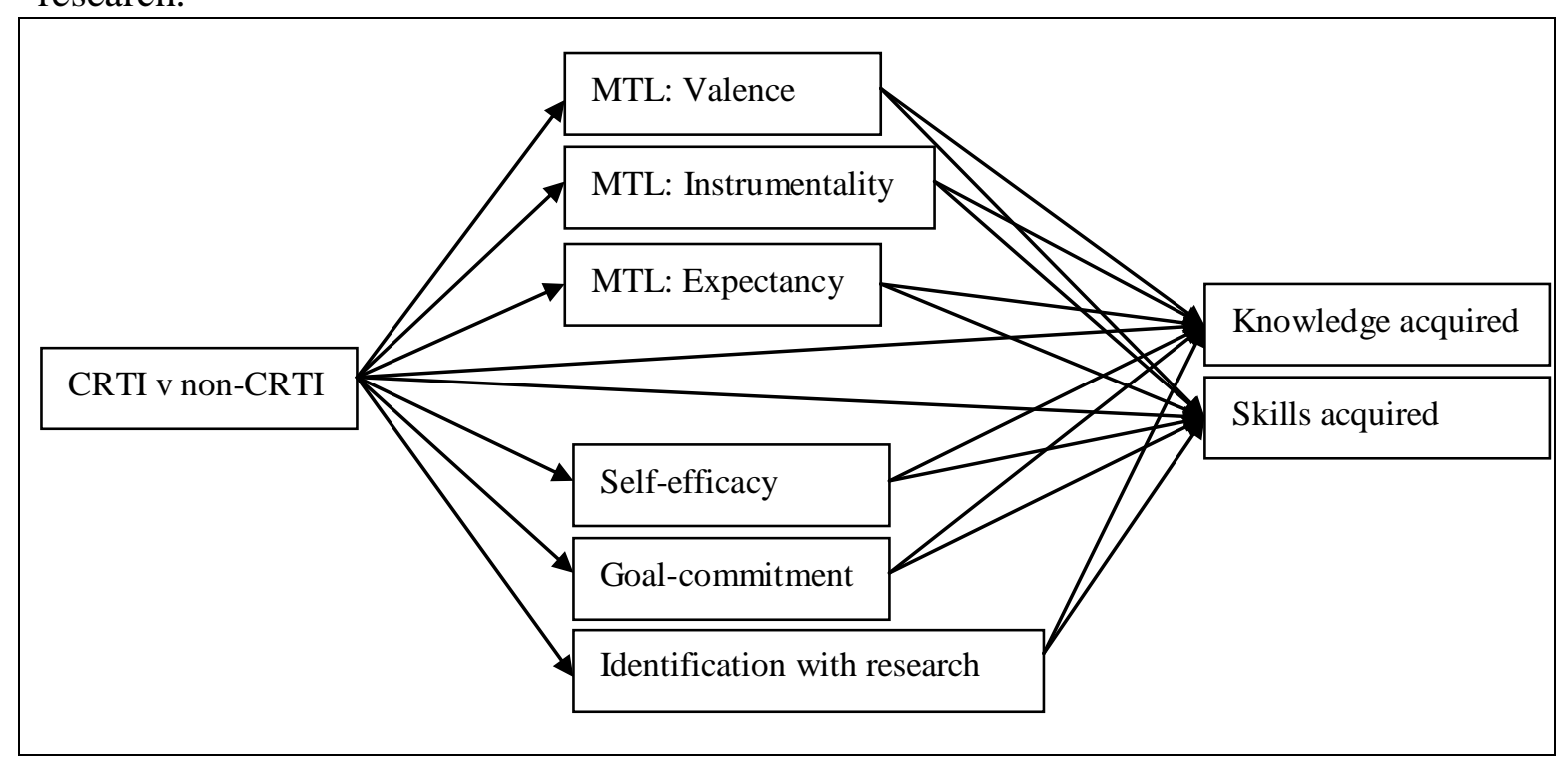




\section{Results}

I conducted four sets of analyses: 1) descriptive statistics, 2) manipulation checks, 3) bivariate correlations to search for confounding variables, and 4) my hypothesis tests.

\section{Descriptive Statistics}

Descriptive statistics were run to provide a frame of reference for group differences and test assumptions of normality (see Table 9). Decisions regarding transformations due to distributional characteristics were made based on visual inspections of histograms, although tests of normality were also run (i.e., Shapiro-Wilk's $W$ ). It has been noted that normality tests, including Shapiro-Wilk's $W$, are overly sensitive and often detect non-normality even when it is not analytically relevant (Fidell \& Tabachnick, 2003).

Table 9. Descriptive statistics.

\begin{tabular}{lccccc}
\hline $\begin{array}{l}\text { Descriptive statistics } \\
\text { Variable }\end{array}$ & Scale & $M$ & SD & Shapiro-Wilks $W$ & Transformation \\
\hline $\begin{array}{l}\text { Focal outcomes } \\
\text { Post-test knowledge }\end{array}$ & $0-14$ & 9.86 & 2.74 & $W=.92, p=.001$ & None \\
Post-test skill & $0-10$ & 6.52 & 3.00 & $W=.90, p<.001$ & None \\
$\begin{array}{l}\text { Affective variables (mediators) } \\
\text { Motivation to learn in training }\end{array}$ & $0-4$ & & & & \\
$\quad$ Valence & & 3.31 & .55 & $W=.74, p<.001$ & Square-root \\
$\quad$ Instrumentality & & 3.21 & .55 & $W=.81, p<.001$ & Square-root \\
$\quad$ Expectancy & & 3.02 & .63 & $W=.85, p<.001$ & Square-root \\
Self-efficacy in research & $0-4$ & 2.60 & .67 & $W=.95, p=.01$ & None \\
$\quad$ Goal-commitment to research & $0-4$ & 2.97 & .61 & $W=.91, p<.001$ & None \\
Identification with research & $0-4$ & 2.44 & .43 & $W=.98, p=.50$ & None \\
\hline
\end{tabular}

\section{Manipulation Checks}

Before using inferential statistics to compare those who were exposed to the CRTI versus those who were not, it was important to evaluate whether 1) individuals were comparable between conditions, 2) training was delivered consistently between conditions and locations, and whether 3) outcomes depended on the trainer's 
improvement over time, and 4) the videos adequately represented culturally responsive material (or not).

Group equivalence. Culturally responsive versus mainstream video priming conditions were compared on age, gender, ethnicity, education, income, pre-training knowledge, pre-training skill, research experience, Native American identity, White identity, sequence, and implementation (i.e., number of activities completed during a training session). The culturally responsive condition had 26 participants, the mainstream condition had 34 participants, and 7 participants viewed neither video prime $(n=67)$. The 7 latecomers who did not view either video prime were given their own code, however I only report comparisons between my groups of interest. Multivariate listwise deletion in the MANOVA reduced the sample size of equivalence testing for both groups (CRTI $n=$ 24; $\sim$ CRTI $n=30$ ). The multivariate $F$ was statistically significant, $F(12,41)=3.53, p=$ $.001, \omega^{2}=.51, B^{9}=.99$, indicating that participants were not equal between conditions

Table 10. Multivariate Analysis of Variance (MANOVA): CRTI on control variables to test group equivalence.

\begin{tabular}{|c|c|c|c|c|c|}
\hline \multicolumn{6}{|c|}{ Manipulation check: Group equivalence } \\
\hline \multirow[b]{2}{*}{ Control variable } & \multicolumn{2}{|c|}{ CRTI } & \multicolumn{2}{|c|}{ Non-CRTI } & \multirow[b]{2}{*}{$F$-statistic, $p$-value } \\
\hline & $M$ & $\mathrm{SD}$ & $M$ & SD & \\
\hline Age & 40.67 & 12.74 & 46.63 & 11.97 & $F(1)=3.13, p=.08 \dagger$ \\
\hline Male & .17 & .38 & .27 & .45 & $F(1)=.75, p=.39$ \\
\hline Education & 2.79 & 1.38 & 3.00 & 1.20 & $F(1)=.35, p=.56$ \\
\hline Income & 3.08 & 1.95 & 4.80 & 1.71 & $F(1)=11.84, p=.001 * * *$ \\
\hline Pre-training knowledge & 8.29 & 2.63 & 8.27 & 2.69 & $F(1)=.001, p=.97$ \\
\hline Pre-training skill & 3.38 & 2.99 & 6.8 & 2.48 & $F(1)=3.66, p=.06 \dagger$ \\
\hline Research experiencet & $-.05 \ddagger$ & $.40 \%$ & $-.04 t$ & $.38 \$$ & $F(1)=.01, p=.92$ \\
\hline Occupational support: & $-.06 \ddagger$ & $.80 \%$ & $-.02 \ddagger$ & $.78 \$$ & $F(1)=.04, p=.84$ \\
\hline Native identity & $-.04 \dagger$ & $.73 \hbar$ & $-.02 \ddagger$ & $.53 \$$ & $F(1)=.02, p=.89$ \\
\hline White identity! & $-.09 t$ & $1.32 \ddagger$ & $.37 t$ & $1.54 \ddagger$ & $F(1)=1.35, p=.25$ \\
\hline Sequence of delivery & 4.29 & 1.71 & 4.9 & 2.04 & $F(1)=1.37, p=.25$ \\
\hline Implementation of activities & 2.58 & .65 & 1.87 & .35 & $F(1)=26.78, p<.001 * * *$ \\
\hline
\end{tabular}

$9 * B=$ statistical power 
Conditions that received the mainstream pre-training prime tended to have higher annual incomes $(M=4.80$ [almost $\$ 50 \mathrm{k}], \mathrm{SD}=1.71)$ than those who received the culturally responsive version $(M=3.08[\$ 30-40 \mathrm{k}], \mathrm{SD}=1.95)$. In addition, not all training sessions completed all of the day's activities at equal rates $(F(1)=26.78, p<$ .001). Training sessions that used the culturally responsive prime condition completed all three activities $65 \%$ of the time and at least two activities $27 \%$ of the time. Training sessions that used the mainstream prime condition completed all three activities $0 \%$ of the time, but did get through at least two of the activities $88 \%$ of the time. One average the mainstream condition only completed 1.87 activities while the culturally responsive condition completed 2.58 activities. Participants in the mainstream prime condition were marginally significantly older in age and marginally significantly higher in pre-training skills. Sequence, or order of delivery, effects were not significant, which does not support the idea that scores were influenced by the trainer's improvement over time.

Video evaluation. To ensure that participants were paying attention and that the videos were equal in all respects, except for culturally responsiveness, a MANOVA was run on the six questions pertaining to the videos' 1) audio quality, 2) picture quality, 3) pace of the video, 4) clarity of the message, 5) relevance to work with participant's community, and 6) cultural appropriateness of the video. Multivariate listwise deletion in the MANOVA reduced the sample size for video evaluations in both groups (CRTI $n=$ 26; CRTI $n=31$ ). The omnibus MANOVA was non-significant, indicating that overall the videos did not significantly differ from one another, $F(6,50)=1.68, p=.15, \omega^{2}=.17$, $B=.59$. Univariate $F$ 's supported the lack of difference between the two videos on all 
five metrics, including, unfortunately, ratings on how culturally appropriate the video was to one's community $(p=.44)$. According to this limited data, trainees did not differentiate between a Native-focused CRTI and a non-Native CRTI video, at least in terms of relevance to work in one's community and the video's cultural appropriateness.

\section{Bivariate Correlations}

Due to sample size restrictions it is necessary to be prudent with the number of covariates that are entered into the final model for hypothesis testing. Therefore correlations were run to identify potential demographic influences on my mediators and dependent variables that need to be accounted for in my model (see Table 11). Dependent variables were knowledge and skill scores at Time 2, mediators included motivation to learn during the training, self-efficacy for research, goal-commitment to research activities, and identification with research. Demographic variables included continuous variables for age, gender, income, education, research experience, occupational support for research, Native American identity, White identity, implementation (i.e., number of activities completed), sequence (i.e., training delivery order), and having missed viewing either prime (i.e., late arrivals). Corrections were made for multiple tests.

Pre-training skills and knowledge were consistently related with pre-training affective motivators and post-training knowledge and skills. Participant gender and education were the next most frequently occurring statistically significant relationships, followed by research experience, occupational support for research, income, Native identity, and implementation (i.e., the number of activities completed). Given the 
information in Table 12, I ran a covariate-inclusive model in addition to a model solely based off of my variables of interest. 
Table 11. Correlation matrix of independent and dependent variables

Correlation matrix of independent and dependent variables, demographics, and control variables

\begin{tabular}{|c|c|c|c|c|c|c|c|c|c|c|}
\hline & Variables & 1 & 2 & 3 & 4 & 5 & 6 & 7 & 8 & 9 \\
\hline 1 & Culturally responsive prime or not & 1 & & & & & & & & \\
\hline 2 & Post-training knowledge & .02 & 1 & & & & & & & \\
\hline 3 & Post-training skill & $-.32 *$ & $.48 * * *$ & 1 & & & & & & \\
\hline 4 & Motivation to learn (valence) & -.16 & $.28^{*}$ & $.44 * * *$ & 1 & & & & & \\
\hline 5 & Motivation to learn (instrumentality) & $-.24 \dagger$ & $.31^{*}$ & $.44 * * *$ & $.88 * * *$ & 1 & & & & \\
\hline 6 & Motivation to learn (expectancy) & -.18 & $.35^{* *}$ & $.44 * * *$ & $.75^{* * *}$ & $.75^{* * * *}$ & 1 & & & \\
\hline 7 & Self-efficacy & .06 & $.37 * * *$ & $.39 * * *$ & .20 & $.22 \dagger$ & $.40 * * *$ & 1 & & \\
\hline 8 & Goal-commitment & -.06 & .13 & $.38 * * *$ & $.33^{* *}$ & $.35^{* * * *}$ & $.46^{* * * *}$ & $.42 * * *$ & 1 & \\
\hline 9 & Identification with research & .01 & $.42 * * *$ & $.49 * * *$ & $.34^{* *}$ & $.34 * *$ & $.37 * * *$ & $.53 * * *$ & $.45^{* * *}$ & 1 \\
\hline
\end{tabular}

Note. $\uparrow p<.10, * p<.05, * * p<.01, * * * p<.005$ 
Table 11b. Correlation matrix of independent and dependent variables, demographics, and control variables continued... Correlation matrix of independent and dependent variables, demographics, and control variables continued...

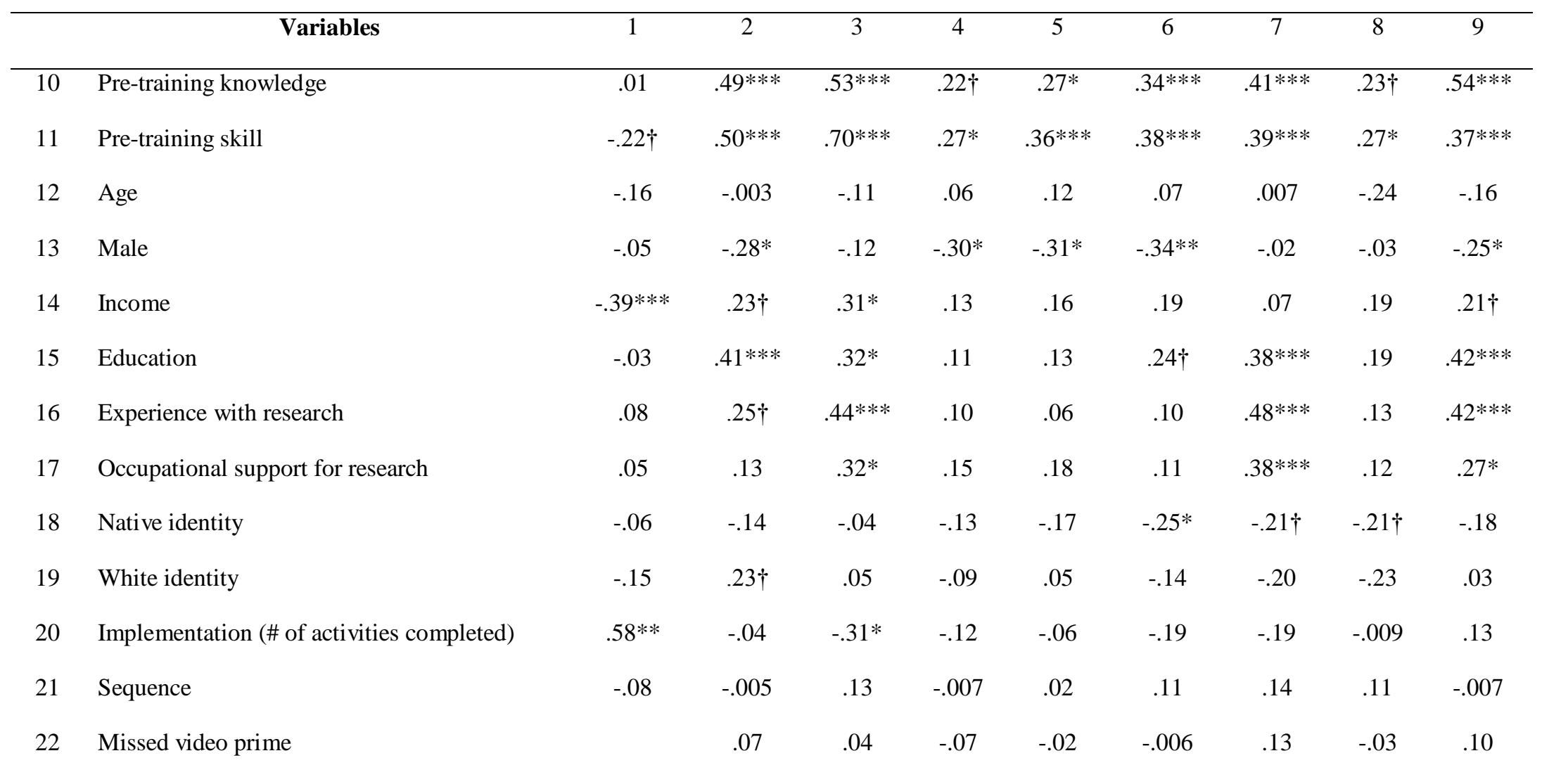

Note. $\dagger p<.10, * p<.05, * * p<.01, * * * p<.005$ 
Table 11c. Correlation matrix of independent and dependent variables, demographics, and control variables continued... Correlation matrix of independent and dependent variables, demographics, and control variables continued...

\begin{tabular}{|c|c|c|c|c|c|c|c|c|c|c|c|c|c|c|}
\hline & Variables & 10 & 11 & 12 & 13 & 14 & 15 & 16 & 17 & 18 & 19 & 20 & 21 & 22 \\
\hline 10 & $\begin{array}{l}\text { Pre-training } \\
\text { knowledge }\end{array}$ & 1 & & & & & & & & & & & & \\
\hline 12 & Age & -.17 & -.07 & 1 & & & & & & & & & & \\
\hline 13 & Male & -.13 & -.09 & .06 & 1 & & & & & & & & & \\
\hline 14 & Income & $.21 \dagger$ & $.36 * * *$ & $.28^{*}$ & .03 & 1 & & & & & & & & \\
\hline 15 & Education & $.54 * * *$ & $.43 * * *$ & .11 & -.04 & $.46^{* * * *}$ & 1 & & & & & & & \\
\hline 16 & $\begin{array}{l}\text { Experience with } \\
\text { research }\end{array}$ & $.47 * * *$ & $.38 * * *$ & $-.28 *$ & .08 & .13 & $.43 * * *$ & 1 & & & & & & \\
\hline 17 & $\begin{array}{l}\text { Occupational support } \\
\text { for research }\end{array}$ & $.34 * * *$ & $.23 \dagger$ & .11 & .05 & .14 & $.30 *$ & $.71 * * *$ & 1 & & & & & \\
\hline 19 & White identity & .11 & .21 & .06 & -.15 & .14 & .21 & -.05 & .002 & $-.21 \dagger$ & 1 & & & \\
\hline 20 & $\begin{array}{l}\text { Implementation (\# of } \\
\text { activities completed) }\end{array}$ & -.09 & .06 & -.07 & -.09 & .04 & -.18 & -.02 & -.13 & .03 & -.14 & 1 & & \\
\hline 21 & Sequence & -.17 & .02 & .14 & .06 & .08 & -.03 & -.20 & -.06 & $.38 * * *$ & .06 & $.21 \dagger$ & 1 & \\
\hline 22 & Missed video prime & $.24 \dagger$ & .01 & .01 & -.06 & .03 & .15 & .19 & .03 & .07 & -.02 & -.09 & $-.31 *$ & 1 \\
\hline
\end{tabular}


Table 12. List of statistically significant relationships between outcome variables, individual differences, and demographics.

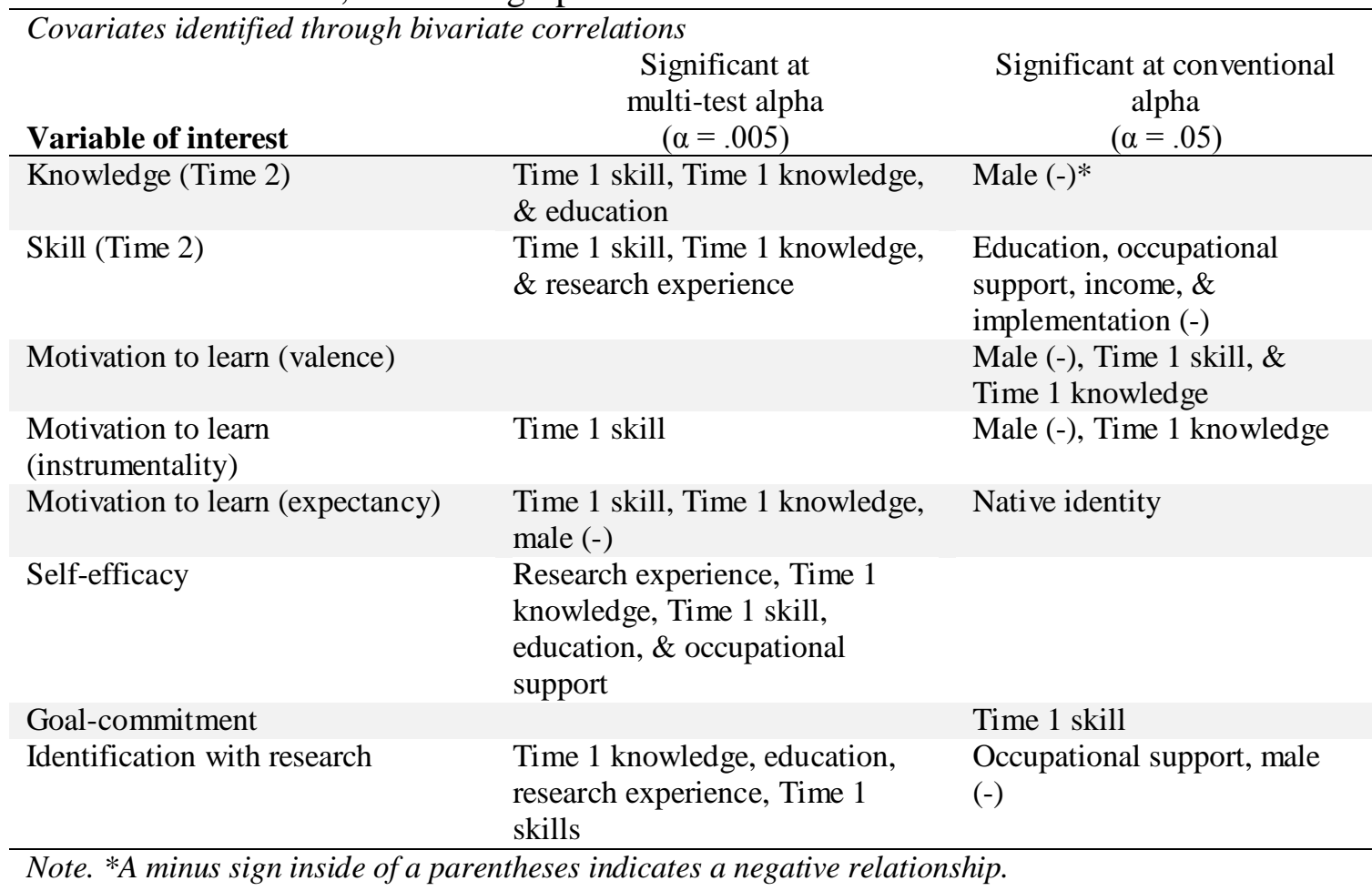

\section{Hypothesis Testing}

To test my hypothesis I ran a structural equation model path analysis where having received a CRTI (i.e., video prime), versus an alternative version, predicted an increase in knowledge and skill scores post-training. In model 1, predictors included prime type (dummy coded) and knowledge and skill scores at pre-test to control for individual differences in KSA's. Outcomes were knowledge and skill outcomes in Time 2 (hypotheses 1-2). Mediators included motivation to learn (i.e., valence, instrumentality, and expectancy subscales), self-efficacy, goal-commitment, and identification with research measured after the video prime but before the training. These were included to test hypotheses 3-6 that a culturally responsive prime would produce an increase in 
Table 13. Coefficient estimates from structural equation model predicting Knowledge and Skill training outcomes from CRTI, controlling for pre-training knowledge and skills, and mediated by affective variables.

\begin{tabular}{|c|c|c|c|c|c|c|}
\hline \multirow{2}{*}{$\begin{array}{l}\text { Model 1: Model without covariates } \\
\text { Predictors }\end{array}$} & \multicolumn{6}{|c|}{ Outcomes } \\
\hline & $\begin{array}{l}\text { Knowle } \\
\text { dge }\end{array}$ & se & $p$-value & Skill & $\mathrm{Se}$ & $p$-value \\
\hline CRTI & -0.33 & .60 & .58 & -.78 & .60 & .19 \\
\hline Pre-test knowledge & .28 & .15 & $.07 \dagger$ & .18 & .15 & .25 \\
\hline Pre-test skill & .20 & .15 & .19 & .51 & .15 & $.001 * * *$ \\
\hline No prime & -.67 & 1.14 & .56 & -.56 & 1.14 & .62 \\
\hline \multicolumn{7}{|l|}{ Affective variables - Direct effects } \\
\hline Motivation to learn (valence) & 2.61 & 1.64 & .11 & 8.91 & 1.64 & $<.000 * * *$ \\
\hline Motivation to learn (instrumentality) & -1.48 & 1.68 & .38 & -1.75 & 1.69 & .30 \\
\hline Motivation to learn (expectancy) & 2.90 & 1.53 & $.06 \dagger$ & -3.90 & 1.54 & $.01 *$ \\
\hline Self-efficacy & .12 & .32 & .69 & .75 & .32 & $.02 *$ \\
\hline Goal-commitment & -.77 & .37 & $.04 *$ & -.47 & .37 & .21 \\
\hline Identification with research & 1.07 & 67 & .108 & 69 & 67 & .30 \\
\hline \multicolumn{7}{|l|}{ Indirect effects } \\
\hline Prime -> valence -> knowledge & -.07 & .14 & .61 & & & \\
\hline Prime -> instrumentality -> knowledge & .06 & .10 & .57 & & & \\
\hline Prime -> expectancy -> knowledge & .007 & .16 & .97 & & & \\
\hline Prime -> self-efficacy $->$ knowledge & .009 & .04 & .82 & & & \\
\hline Prime -> goal commit -> knowledge & -.15 & .19 & .43 & & & \\
\hline Prime -> identification -> knowledge & -.009 & .14 & .95 & & & \\
\hline Prime -> valence -> skill & & & & -.25 & .47 & .59 \\
\hline Prime -> instrumentality -> skill & & & & .07 & .11 & .54 \\
\hline Prime -> expectancy -> skill & & & & -.01 & .22 & .97 \\
\hline Prime -> self-efficacy -> skill & & & & .06 & .20 & .79 \\
\hline Prime -> goal commit -> skill & & & & -.09 & .13 & .48 \\
\hline Prime -> identification -> skill & & & & -.006 & .09 & .95 \\
\hline
\end{tabular}

Note. $\dagger \alpha=.10, * \alpha=.05, * * \alpha=.01, * * * \alpha=.005$

affect-related motivators, and that this increase would partially explain (i.e., mediate) the relationship between culturally responsive materials and improved knowledge (hypothesis 7) and skill (hypothesis 8) scores. In model 2, the same model was tested again controlling for the potentially confounding variables identified in Table 12. SEM requires no missing data across all variables in the dataset. Listwise deletion lowered the final sample size $(N=50)$. None of my hypotheses were supported in either model (see 
Tables 13 - 16). CRTI was not significantly related to knowledge, skill (see Tables 13 and 15), or affective outcomes (see Tables 14 and 16). Consequently, none of the indirect effects were significant for CRTI's effect on knowledge or skill. A post-hoc power analysis using the effect sizes from my CRTI intervention ranged between $\eta^{{ }^{2}}=.01^{10}$ for knowledge acquisition and $\eta^{{ }^{2}}=.04$ for skill acquisition, controlling for covariates. Cohen (1988) describes these as a small to medium effects. An a priori power analysis with these effect sizes estimates that, to achieve adequate power, replication studies would need samples sizes of $\mathrm{N}=620$ to $\mathrm{N}=102$.

Table 14. Direct effects of CRTI on affective variables purposed to mediate its effect on learning outcomes.

Model.1: Model without control variables

\begin{tabular}{llll} 
Affective variables as outcomes & Culturally responsive prime & Se & $p$-value \\
\hline
\end{tabular}

$\begin{array}{lccc}\text { Motivation to learn (valence) } & -.03 & .05 & .59 \\ \text { Motivation to learn (instrumentality) } & -.04 & .05 & -.45 \\ \text { Motivation to learn (expectancy) } & .002 & .06 & .97 \\ \text { Self-efficacy } & .07 & .27 & .79 \\ \text { Goal-commitment } & .20 & .23 & .39 \\ \text { Identification with research } & -.009 & .13 & .95\end{array}$

Note. $\dagger \alpha=.10, * \alpha=.05, * * \alpha=.01, * * * \alpha=.005$

Direct effects of affective mediators on knowledge and skill acquisition were observed (see Table 13 and 15). In model 1, knowledge acquisition was negatively predicted by goal-commitment $(\beta=-.77, p=.04)$ and positively predicted by motivation to learn expectancy, albeit with only marginal significance $(\beta=2.90, p=.06)$. Skill acquisition was predicted by motivation to learn valence $(\beta=8.91, p<.001)$ and selfefficacy $(\beta=.75, p=.02)$. Motivation to learn expectancy was also significant, although in the opposite direction than hypothesized $(\beta=-3.90, p=.01$; see Figure 4$)$.

${ }^{10} \eta^{2}=$ partial eta squared 
Figure 4. Model 1 results: Without covariates entered

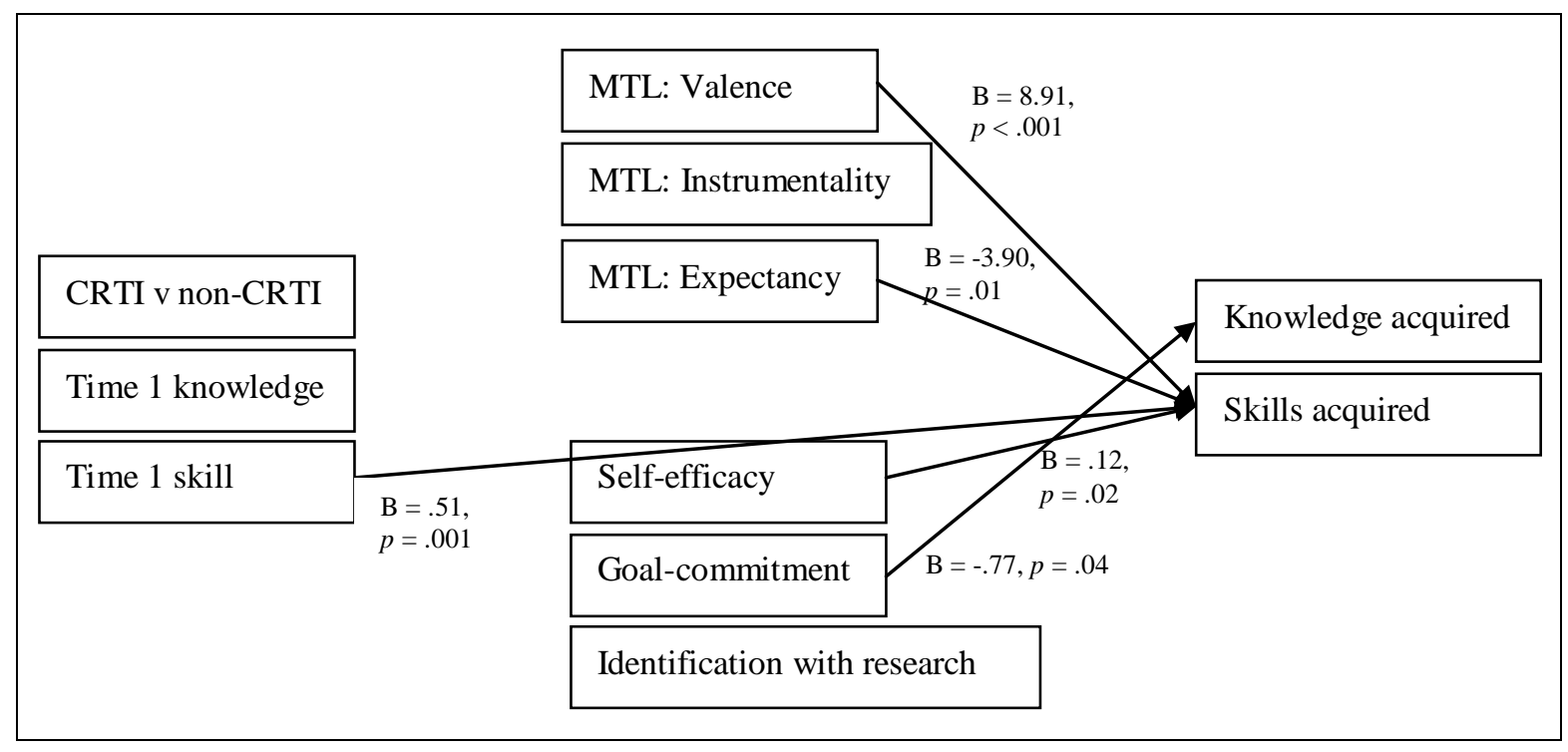

In model 2, controlling for covariates, the relationships between knowledge acquisition and goal-commitment and motivation to learn expectancy disappeared (compare Figures 4 and 5). The positive relationships between skill acquisition and motivation to learn valence $(\beta=9.39, p<.001)$ and self-efficacy $(\beta=.61, p=.04)$ remained, however, so did its negative relationship with motivation to learn expectancy $(\beta=-5.26, p<.001 ;$ see Table 15$)$. 
Table 15. Coefficient estimates from structural equation model predicting Knowledge and Skill training outcomes from CRTI, controlling for pre-training knowledge and skills, and mediated by affective variables. Control variables included.

\begin{tabular}{|c|c|c|c|c|c|c|}
\hline \multirow{2}{*}{$\begin{array}{l}\text { Model 2: Model with covariates } \\
\text { Predictors }\end{array}$} & \multicolumn{6}{|c|}{ Outcomes } \\
\hline & $\begin{array}{l}\text { Knowled } \\
\text { ge }\end{array}$ & se & $p$-value & Skill & $\mathrm{Se}$ & $p$-value \\
\hline CRTI & -.09 & .55 & .87 & .32 & .66 & .62 \\
\hline Pre-test knowledge & .29 & .15 & $.05^{*}$ & .24 & .14 & $.096 \dagger$ \\
\hline Pre-test skill & .28 & .14 & $.04 *$ & .60 & .13 & $<.000 * * *$ \\
\hline No prime & -.55 & 1.12 & .62 & .37 & 1.13 & .75 \\
\hline \multicolumn{7}{|l|}{ Affective variables - Direct effects } \\
\hline Motivation to learn (valence) & 2.42 & 1.66 & .15 & 9.39 & 1.52 & $<.000 * * *$ \\
\hline Motivation to learn (instrumentality) & -1.21 & 1.71 & .48 & -2.31 & 1.56 & .139 \\
\hline Motivation to learn (expectancy) & 1.98 & 1.59 & .21 & -5.26 & 1.41 & $<.000 * * *$ \\
\hline Self-efficacy & -.01 & .30 & .97 & .61 & .29 & $.04 *$ \\
\hline Goal-commitment & -.64 & .36 & $.08 \dagger$ & -.11 & .34 & .74 \\
\hline Identification with research & .94 & .68 & .17 & .47 & .63 & .46 \\
\hline \multicolumn{7}{|l|}{ Affective variables - Indirect effects } \\
\hline CRTI prime -> valence -> knowledge & .06 & .26 & .81 & & & \\
\hline $\begin{array}{l}\text { CRTI prime -> instrumentality -> } \\
\text { knowledge }\end{array}$ & .01 & .30 & .97 & & & \\
\hline $\begin{array}{l}\text { CRTI prime -> expectancy -> } \\
\text { knowledge }\end{array}$ & .32 & .49 & .51 & & & \\
\hline $\begin{array}{l}\text { CRTI prime -> self-efficacy -> } \\
\text { knowledge }\end{array}$ & $<.001$ & .01 & .97 & & & \\
\hline $\begin{array}{l}\text { CRTI prime -> goal commit -> } \\
\text { knowledge }\end{array}$ & .03 & .03 & .37 & & & \\
\hline $\begin{array}{l}\text { CRTI prime -> identification -> } \\
\text { knowledge }\end{array}$ & -.02 & .15 & .63 & & & \\
\hline CRTI prime -> valence -> skill & & & & .25 & 1.00 & .81 \\
\hline CRTI prime -> instrumentality -> skill & & & & .02 & .56 & .97 \\
\hline CRTI prime -> goal commit -> skill & & & & -.86 & 1.12 & .45 \\
\hline CRTI prime -> self-efficacy -> skill & & & & -.02 & .03 & .46 \\
\hline CRTI prime -> goal commit -> skill & & & & .005 & .02 & .75 \\
\hline CRTI prime -> identification $->$ skill & & & & -.01 & .03 & 67 \\
\hline \multicolumn{7}{|l|}{ Control variables } \\
\hline Past experience with research & - & - & - & 1.04 & .98 & .29 \\
\hline Occupational support for research & & & & -.14 & .40 & .72 \\
\hline Education level & .20 & .24 & .42 & -.16 & .25 & .53 \\
\hline Male & -.25 & .75 & .74 & - & - & - \\
\hline Income & - & - & - & -.10 & .15 & .52 \\
\hline Implementation (\# of activities) & - & - & - & -1.43 & .49 & $.004 *$ \\
\hline
\end{tabular}

Note. $\dagger \alpha=.10, * \alpha=.05, * * \alpha=.01, * * * \alpha=.005$ 
Table 16. Direct effects of CRTI on affective variables purposed to mediate its effect on learning outcomes.

Model.2: Model with control variables

\begin{tabular}{lccc} 
Affective variables as outcomes & Culturally responsive prime & Se & $p$-value \\
\hline Motivation to learn (valence) & -.04 & .05 & .43 \\
Motivation to learn (instrumentality) & -.05 & .04 & .30 \\
Motivation to learn (expectancy) & -.02 & .05 & .61 \\
Self-efficacy & -.009 & .24 & .97 \\
Goal-commitment & .16 & .21 & .44 \\
Identification with research & .03 & .11 & .81
\end{tabular}

Note. $\uparrow \alpha=.10, * \alpha=.05, * * \alpha=.01, * * * \alpha=.005$

Figure 5. Model 2 results: With covariates entered

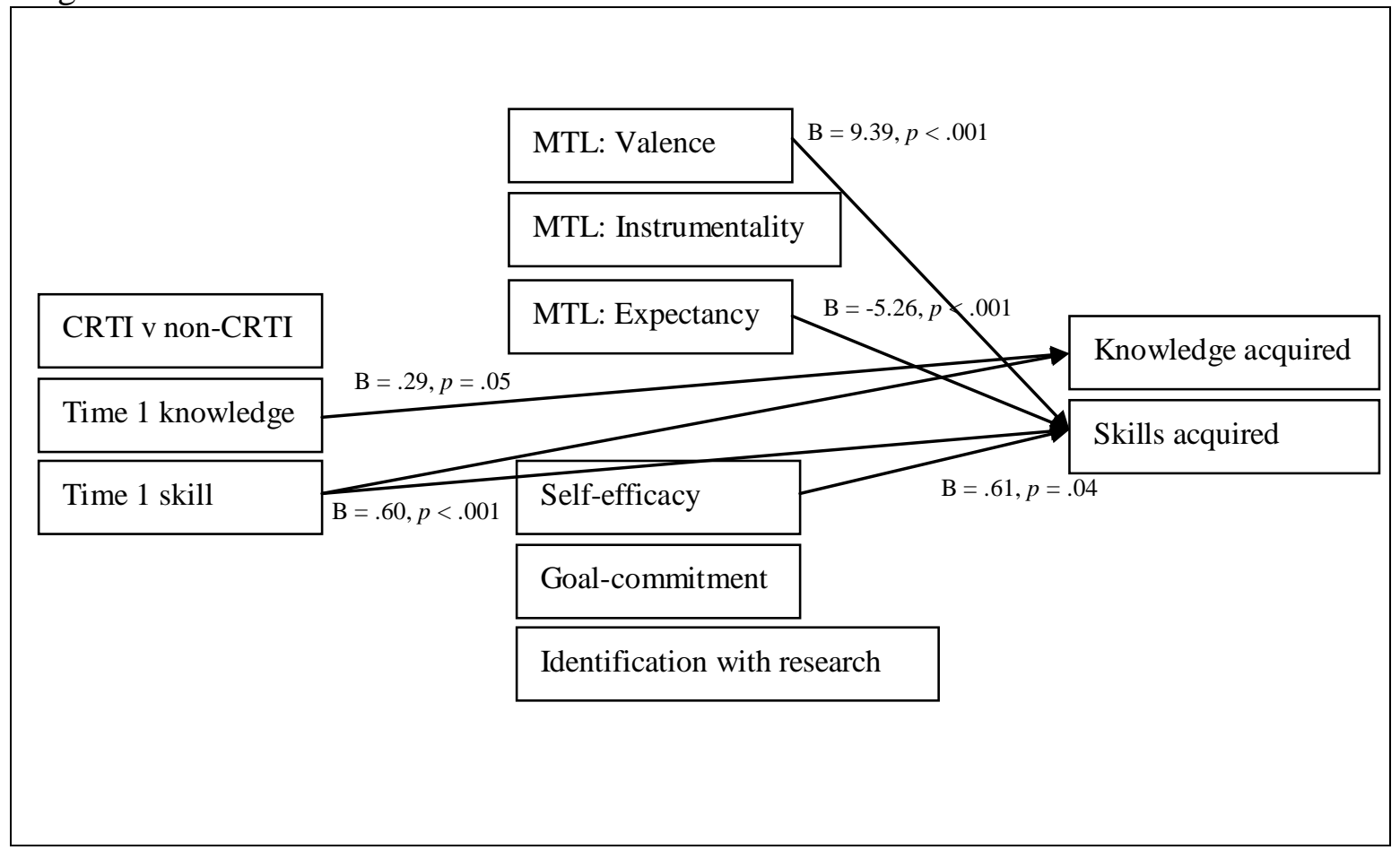

\section{Supplementary Analyses}

To evaluate alternative explanations as to why my hypotheses were not supported I re-ran the manipulation check MANOVA that compared video evaluation scores on community relevance and cultural appropriateness, except I added the interaction 
between prime type and ethnic group, which I re-defined Natives alone, White alone, Native and White mixed, and other ethnicities.

Ethnicity and priming effects. Neither Prime type or Ethnic group had significant main effects, however a significant interaction showed that ratings on a video's community relevance, $F(3,48)=3.65, p=.02$, and culturally appropriateness, $F(3,48)=3.93, p=.01$, depended on one's ethnic grouping, multivariate $F(6,96)=2.64, p=$ .02. Natives alone $\left(M_{a l t}=2.11, M_{c r t i}=1.64\right)$, Whites $\left(M_{a l t}=2.33, M_{c r t i}=2.00\right)$, and other ethnicities $\left(M_{a l t}=3.00, M_{c r t i}=2.00\right)$ all rated the alternate version (i.e., with white researchers only) as more relevant to their community than the culturally responsive prime. Similarly, Whites $\left(M_{a l t}=2.33, M_{c r t i}=1.50\right)$ and other ethnicities $\left(M_{a l t}=2.00, M_{c r t i}\right.$ $=3.00$ ) rated the alternate version higher on cultural appropriateness, this time Natives alone rated each video near equal $\left(M_{a l t}=1.67, M_{c r t i}=1.64\right)$. On the other hand Native/White mixed individuals rated the culturally responsive video prime as more relevant $\left(M_{a l t}=1.50, M_{c r t i}=2.40\right)$ and more appropriate $\left(M_{a l t}=1.38, M_{c r t i}=2.20\right)$ than the alternative version.

Additionally, using a MANCOVA that compared knowledge and skill scores at time 2 by prime type, ethnic group, and their interaction, controlling for scores at time 1 , Native/White mixed individuals scored higher on average than Natives or Whites alone in the culturally responsive prime condition but not in the alternative mainstream prime condition, although these differences were not significant. It is possible that although this prime did not get the reaction I expected from my sample as a whole, for mixed individuals the culturally responsive video resonated. One finding from my structural 
equation model (not reported) that lends credibility to this idea were the significant negative correlations with motivation to learn: expectancy (i.e., expectancy to learn material $)$ and both Native $(\beta=-.07, p=.05)$ and White identity $(\beta=-.04, p=.02)$.

Apparently the more one identified with either Native or White culture, the less that they expected to learn from the training. I interpret this as meaning that the training appealed more to the middle of the training participants versus those in the poles with regard to ethic identity.

Video evaluations as predictors. Participant ratings of my video manipulation did not significantly differentiate between community relevance and cultural appropriateness. With the belief that both videos were somehow equally relevant and appropriate, I wanted to see if those elements of the videos predicted outcomes given that relevance and appropriateness are both considered key ingredients to CRE. I re-ran Model 1 two more times, once with video appraisals of cultural appropriateness instead of CRTI as the predictor, and once with community relevance instead of CRTI as the predictor.

Cultural appropriateness significantly predicted motivation to learn valence $(\beta=$ $.08, p=.01)$, instrumentality $(\beta=.07, p=.02)$, and expectancy $(\beta=.13, p<.001)$, selfefficacy $(\beta=.43, p=.01)$, and goal-commitment $(\beta=.32, p=.03)$ and marginally predict skill score increases $(\beta=.93, p=.08)$. In addition, pre-training videos' cultural appropriateness had significant indirect effects on Time 2 skill scores through valence (appropriateness $\rightarrow$ motivation to learn: valence $\rightarrow$ skill; $\beta=.67, p=.03$ ) and expectancy (appropriateness $\rightarrow$ motivation to learn: expectancy $\rightarrow$ skill; $\beta=-.73, p=.01$ ). 
Responsive Training 110

Community relevance significantly predicted skill-score increases $(\beta=1.03, p=$ $.05)$, motivation to learn valence $(\beta=.09, p=.005)$, instrumentality $(\beta=.09, p=.003)$, and expectancy $(\beta=.12, p<.001)$, self-efficacy $(\beta=.43, p=.01)$, and goal-commitment $(\beta=.37, p=.007)$. In addition, pre-training videos' relevance had significant indirect effects on Time 2 skill scores through valence (relevance $\rightarrow$ motivation to learn: valence $\rightarrow$ skill; $\beta=.73, p=.02$ ) and expectancy (relevance $\rightarrow$ motivation to learn: expectancy $\rightarrow$ skill; $\beta=-.61, p=.02$ ). Video relevance to one's culture also revealed one marginally significant indirect effect on knowledge scores through valence (relevance $\rightarrow$ motivation to learn: valence $\rightarrow$ knowledge; $\beta=-.32, p=.098)$. 


\section{Discussion}

This study failed to show that training effectiveness can be enhanced by incorporating culturally responsive features in a pre-training video. I was unable to show that a culturally responsive prime intervention yielded an increase in knowledge and skills over an equivalent pre-training prime intervention without the cultural component. I was also unable to show that motivation to learn, self-efficacy, goal-commitment, and identification with research were higher at the outset for participants who received a culturally responsive prime. Finally, this study failed to show that the effect of a culturally responsive prime on knowledge and skills occurs because of an effect on training-related affective variables (e.g., motivation to learn, self-efficacy, goalcommitment, and identification with research).

In Model 1, goal-commitment predicted a decrease in knowledge scores, but this effect disappeared after adding covariates in Model 2. Across both models, significant positive results on skill acquisition, but not knowledge acquisition, were discovered for motivation to learn valence and self-efficacy, while significant negative results were found for motivation to learn expectancy. These findings extend other research in training (Colquitt et al., 2000) that emphasize the importance of valence and self-efficacy to the learning process, specifically in skill acquisition.

The initial reaction from this data is to conclude that I did not find evidence for the effect of a CRTI. However, given my manipulation check failing to distinguish between conditions in terms of relevance and cultural appropriateness, what this really shows is a failure to properly capture culturally responsive pre-training video content that 
is at the same time comparable to a non-culturally responsive video alternative. This can be viewed in at least two ways: one that emphasizes what my manipulated variable did not include and one that emphasizes what this study contributes to our understanding of cultural responsiveness.

With regard to what my independent variable did not include, it is important to distinguish the 13-minute pre-training intervention that I labeled as "culturally responsive" from other educational approaches that incorporate TEK or indigenous ways of knowing (Barnhardt \& Kawagley, 1998, 2005; James, 2001; Riggs, 2007). My intervention was culturally responsive in that it emphasized the presence of similar others (i.e., Native researcher interviews v. non-Native research interviews) and the connection between research and the potential to contribute to one's community (i.e., personal relevance and collectivistic motivation). However the content was essentially that of a college-level social science methods course with an emphasis on education grants and program evaluation and directed at a Pan-Indian audience. A TEK-based culturally responsive approach would be much more substantial in its integration of Native science (e.g., traditional astronomy, medicine, zoology, botany) and culture (e.g., language) and involve multiple levels of change. Barnhardt (2002) discussed how educational communities were becoming culturally responsive in Alaska, but to do so had to discuss the complex historical interaction between policy, educational institutions, and tribal communities, where favorable policies, school administrators, teachers, curriculum writers, students, families, and tribal organizations all contributed to this change, sometimes one taking the lead more than others. 
My definition of culturally responsiveness fell more in line with that of Klug and Whitfield (2003), in their emphasis on real-life relevance and the use of student backgrounds to facilitate learning, than Demmert and Towner's (2003), who stress the inclusion of Native language, spirituality, inter-generational involvement in teaching and learning, traditional pedagogy, and cultural etiquette. Perhaps had I better emphasized the overlap between mainstream science and Indigenous Ways of Knowing, as prescribed in Barnhardt and Kawagley, 2005), there would have been a stronger effect of the intervention.

Figure 6. Qualities Associated with Traditional Knowledge and Western Science. Reprinted from "Indigenous Knowledge Systems/Alaska Native Ways of Knowing," by R. Barnhardt and A.O. Kawagaley, 2005, Anthropology and Education, 36, p. 8. Copyright 2005 by American Anthropological Association.

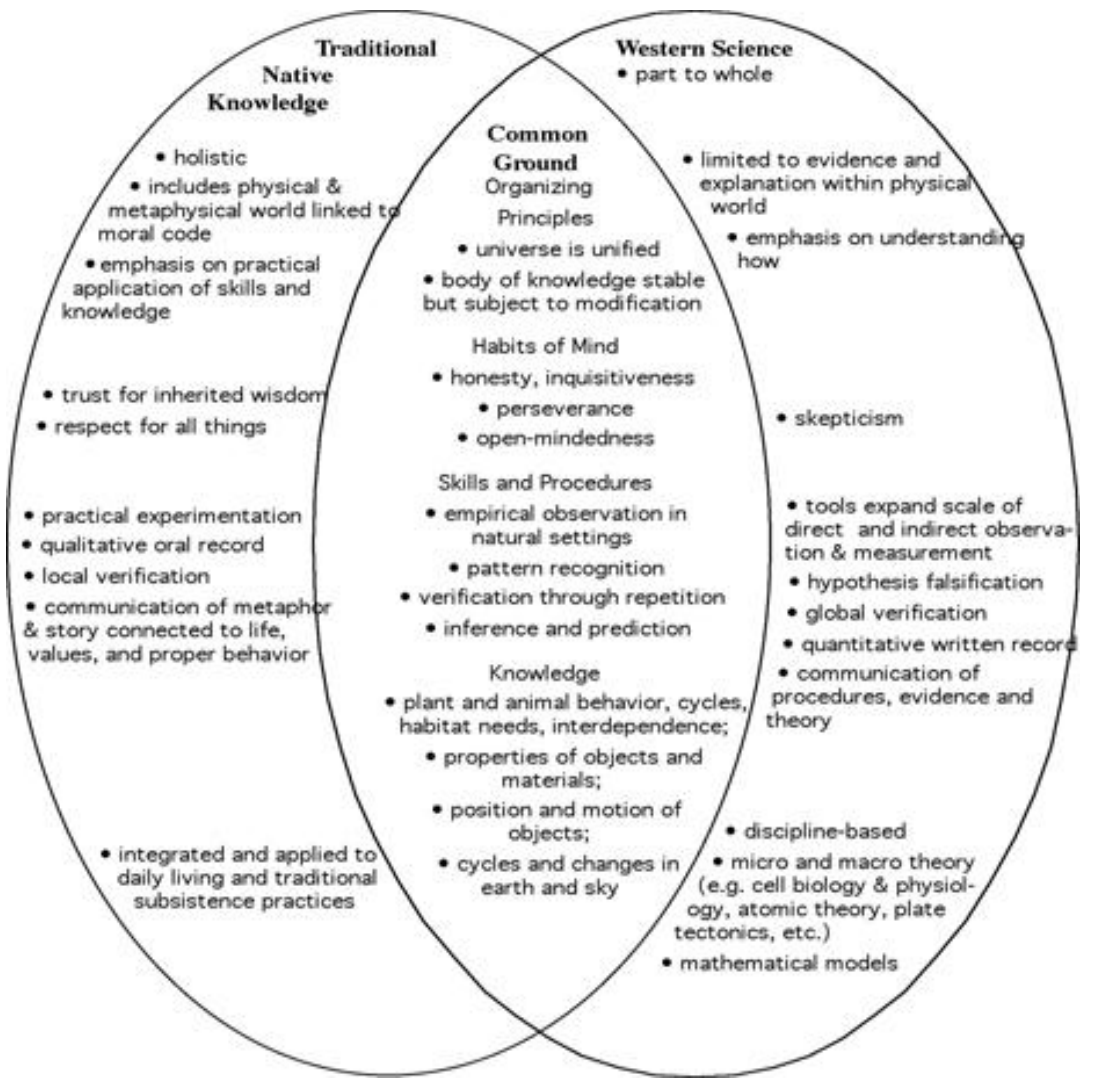


With this point of view I could evaluate my findings from the perspective that my CRTI condition was not culturally responsive enough, or that my non-CRTI condition was too culturally responsive. This leads to the empirical question of "not culturally responsive enough for whom?" Supplementary analyses revealed that Natives alone (i.e., non-mixed ethnicity) rated the videos with White researchers as more relevant and equally appropriate. This is interesting but is not totally unexpected. In 2004 when I was doing research with a reservation in the southwest, I asked the resident psychologist about the complete lack of Native medical personnel at their Health and Wellness Center. She informed me that many Native doctors experience a form of internalized racism from the community, where their education and credentials are deemed less trustworthy or valuable as White doctors. When individuals do this to themselves the phenomenon has been referred to as "Stereotype threat" (Steele \& Aronson, 1995), however in this context a self-stereotype was applied to another in-group member. It is possible that these ratings captured this dynamic. Results from supplementary analyses further support this conclusion given that ethnically-mixed individuals, who presumably identify with some aspects of both cultures, received the most from the training experience.

Another way to view my findings is in terms of the contributions it has made to culturally responsive approaches. As before, I would argue that it was my video manipulation that failed to concentrate cultural responsiveness more than the alternative version, such that a model that compares the two conditions fails to find an effect. However, this is still different than whether the ingredients of culturally responsiveness were effective. I did not add the manipulation check variables to my SEM models due to 
the fact that my prime conditions did not significantly differ on any of the comparison metrics. However, I ran my structural equation Model 1 two more times as supplementary analyses, but replaced prime type (i.e., CRTI) as a predictor with either the pre-training videos' culturally appropriateness or community relevance ratings as predictors instead. Cultural appropriateness and relevance predicted increases in motivation to learn valence, instrumentality, expectancy, self-efficacy, and goalcommitment. These findings suggest that while my video interventions did not differ in cultural responsiveness with regard to relevance and cultural appropriateness, that those key ingredients of cultural responsiveness are still driving learning outcomes. This is in line with definitions of culturally relevant teaching by CRE pioneers that focus on relevance (Ladson-Billings, 1995) and cultural competence (Gay, 2000). It might also suggest that materials do not have to be tailored to specific a cultural group or use culturally-tied symbols to be culturally responsive.

\section{Implications}

This study has implications for theory and research in training and education, particularly within the contexts of globalization, underrepresentation, and Native American populations. Although I did not demonstrate the heightened effectiveness of CRTI over a more typical pre-training intervention, I did advance the dialogue with regard to theory and research on culturally responsive approaches in at least two ways. First, by controlling for individual differences, randomizing conditions, and measuring pre- and post-training, this study utilized one of the most controlled quasi-experimental designs in culturally responsive education research to date. This responded to the long 
awaited call from Demmert and Towner (2003) who lamented the lack of rigorous designs in this field.

Second, this study showed that materials can be deemed culturally appropriate and community relevant even when they include racially dissimilar others and the information is not framed specific to one cultural community, although this could be moderated by ethnic group composition and impacted by stereotype threat. The fact that many participant's already held jobs where research and statistics are used make it difficult to generalize. A less complex training experience with a more representative population would help to bring clarity to the picture.

Since I did not achieve statistical significance for any of my eight hypotheses, I am not able to comment on the viability of culturally-bound ATIs (Cronbach \& Snow, 1977) or the use of Compatibility theory (Tharp \& Gillmore, 1989), Cultural-HistoricalActivation-Theory (CHAT) and Cognitive Theory (Tharp, Estrada, Dalton, \& Yamauchi, 2000) as explanatory frameworks. However, supplementary analyses did demonstrate the utility of using pre-training primes that frame the training objectives in terms of their appropriateness and relevance to work in one's cultural community. Motivation to learn, self-efficacy, and goal-commitment all increased significantly depending on perceptions of cultural appropriateness and relevance of the prime. This supports the advice from Cannon et al. (1998) on pre-training priming and adds to the our knowledge of predictors of motivation (Colquitt et al., 2000; Major, Turner, \& Fletcher, 2006; Smith-Jentsch, Jentsch, Payne, \& Salas, 1996) and self-efficacy (Baldwin \& Magjuka, 1997; Blume, Ford, \& Baldwin, 2010). 


\section{Applications}

Findings from this study are applicable to training practices in direct and indirect ways. Directly, this study showed pre-existing knowledge and skills are important in whether or not new research knowledge and skills are acquired. This finding testifies to the value of needs assessments to identify who needs what type of training. Participants in these training ranged in education level from less than an $8^{\text {th }}$ grade education to $\mathrm{PhDs}$, and it was difficult to design a training that was both accessible as well as challenging to everyone. Separate training for different knowledge and skills levels might be useful in building learning readiness for advanced concepts.

Another direct application from this study is the finding that valence, instrumentality, and expectancy forms of motivation to learn can differentially affect learning outcomes. Valence was the only form that positively related to learning outcomes while the other two were either non-significant or predicted decreases in skill acquisition. The negative relationship between expectancy and skill acquisition was unexpected. The more participants thought they would be able to master training information (i.e., expectancy) the worse they did on post-test skill assessments. It was almost as though heightened motivation in this forms created a false sense of security that resulted in lower processing of the information or, alternatively, a sense of being overwhelmed by so much new and abstract information (e.g., skill-based answers that were correct in the pre-test were often wrong in the post-test). This may be a problem specific to science and research. 
In a program evaluation I did for Portland State University's Center for Climate and Aerosol Research in 2014, in contrast to our expectations, a 10-week long internship involving lab and field work decreased intrinsic motivation, self-efficacy, and identification with research. I suggested in that evaluative report that preconceptions about research and science may be confronted with the difficult and/or mundane activities of scientific research and have a deflating, albeit sobering, effect. My findings might suggest trainers should explain that research is a life-long learning process and that the belief that one will be qualified or able to conduct rigorous research at the end of a oneday training is hopeful but ultimately misguided. Trainers should encourage the desire to learn for understanding-sake instead to increase valence.

A third application of this data comes from the supplementary analysis in the discussion above pertaining to priming appropriateness and relevance cross-culturally. Although it was not in my hypotheses, the data showed that when a pre-training video was viewed as culturally appropriate and relevant to ones' work in their community, that it had a positive effect on skill acquisition by increasing the valence and self-efficacy. Since cultural appropriateness and relevance are two of the major tenets of CRE, manipulations that increase these perceptions for people of particular cultural groups could be considered approaching culturally responsiveness. In this case, showing a video of White researchers working on societal problems can still be effective with Native researchers as long as the information is relevant and culturally appropropriate.

Ladson-Billings (1995) also calls for the development of a critical consciousness and a focus on learning outcomes, and Gay (2000) and Pewewardy and Hammer (2003) 
advocate the importance of a safe, non-discriminating learning environment and cultural competence of instructors as well. This study could not provide any evidence with regard to these other factors, but in terms of cultural appropriateness and relevance this study supports their importance as pre-conditions to learning. A word of caution came from the negative indirect effects of relevance to expectancy to skill outcomes. Although relevance increased expectancy, expectancy predicted decreases in skill acquisition. Messages or primes that increase the relevance of learning the subject matter to one's life and work need to be tempered with explanations of the difficulty of the material and the likelihood of transfer opportunities (Baldwin \& Ford, 1988). Alternatively, over-confidence may have caused individuals not to try as hard since they felt they already understood the material and were not going to get anything out of the exit survey.

Indirectly this study applies to training in practice by offering an example of a science-related program that was requested, designed, implemented, and evaluated by and for Native Americans. Although skill scores did not increase significantly over the course of the training $(p=.91)$, knowledge increased $(p<.001)$ and subjectively $98 \%$ of those who filled out the post-test survey reported that the training was useful. When asked if they were interested in similar training on six related subjects (e.g., qualitative methods, statistical analyses, research issues in Indian Country) over three quarters (76\%) said they would be interested in all six training modules. Despite the lack of hypothesized effects of my independent variable, the training itself should be seen as progress for equipping underrepresented communities and supporting indigenous sovereignty through research-related capacity building. 


\section{Limitations}

Although this study is arguably the most rigorous project on culturally responsive training to date, it is not without its limitations. First, a stronger and more power design would be to randomly assign participants to different conditions. In this case, it was only possible to randomly assign training conditions by location. The low number organizational affiliates also restricted plans for more complex designs (e.g., Solomon Four Groups Design).

Second, participants in this training were members of a volunteer-based nonprofit organization and not the workplace of trainees. Therefore data on the effectiveness of the training did not include any objective measures of increased capacity or skill on the job. In addition, because participants were voluntary members rather than employees, the range in participant education and skill level forced the content to be fairly introductory. The effect of CRTI may have been attenuated with such conceptual, versus applied, information.

Third, it would have been ideal to have separate training altogether, one that included a culturally responsive approach and one that did not, rather than offer the same training with such a short intervention. My culturally responsive component was separated from the essential training content to eliminate researcher expectancy effects (i.e., the Pygmalion effect, Kierein \& Gold, 2000) but still allow all participants to undergo the complete training for practical reasons. As a research design this separation was useful, however another major tenet of CRE (i.e., adaptive pedagogy) could not be tested because of it. The training was still taught primarily through lecture in a 
hierarchical, cognitively-focused, top-down approach, which is in direct contrast to the more horizontal, experiential, collaborative approach called for by most culturally responsive educators of Native Americans (Pewewardy, 1998, 2012; Pewewardy \& Fitzpatrick, 2009). In the end this design only evaluated a "culturally responsive" training prime (Cannon-Bowers et al., 1998) in the context of a complex all-day training and not a full representation of cultural responsiveness in action. Although priming effects are generally found to have an effect (Van den Bussche, Noortgate, \& Reynvoet, 2009), as a test of CRTI this manipulation may have been too weak and brief compared to other components of the training.

Fourth, a limitation to the conclusion to this study is that the instructor was not able to remain blind to the video intervention. On four occasions there were technical difficulties that required the instructor to re-enter the room after the video had started, on two occasions a trainee mentioned the content to the instructor that gave away the prime type, and on one occasion that at first seemed to go successfully, I could hear the music that was indicative of a particular prime through the wall. Perhaps the non-significant relationship between the prime type and the outcomes makes this point moot; however it should be acknowledged that I cannot rule out the possibility that my knowledge of the prime type for each location influenced my delivery.

Fifth, there were elements to the training content that may have diluted the effect of the prime conditions. Although pre-training video evaluations were not statistically different it is possible that the culturally-framed video would have shown effects had the training been completely generic. As it was, the trainer was Native American, the data 
used in skill assessment pertained to Native students, the majority of attendees were Native American, and it was promoted and affiliated with a well-known Native organization in the state. Questions and discussion often related to indigenous or Native American issues and topics, and a reoccurring theme in initial introductions was feelings of resistance to Western science and feelings of being forced to convert Native experiences into numbers for the government to then deny funding. Although the slides, activities, materials, format, and delivery were all the same throughout the bulk of the training, both conditions were followed by culturally responsive elements in-training which may have diluted the effect of the pre-training prime.

\section{Future Research}

In addition to more and stronger designs that address the limitations noted above, future research on CRTI should include more studies specific to the training as well as to the trainee/trainer characteristics. Training-specific research is needed to assess the prevalence, dosage, objective type, validity, and organizational support of CRTI. Trainee/trainer-related research is required to evaluate the role of group composition, cultural identification, and instructor-trainee similarity.

Training-specific research. Research on the prevalence of CRTI is important to recognize popular trends, comparative, and correlational studies. It is likely that CRTI designs are widespread, even if they are not offered under the label of CRTI. For instance, since culture is often invisible to its proprietor, organizations that are owned by, operated by, and employ members of the same cultural group will probably offer a CRTI design without considering it a special way of conducting training. This is probably also 
the case for branches that are primarily staffed by a particular cultural group, in which general policies are translated into local practices. Finally, some variant of CRTI probably takes place in organizations that are particularly responsive on their own accord, such as companies that offer training for customer service in ethno-cultural communities. Identification of companies already using culturally responsive designs would allow for comparisons between organizations, and could provide a cultural insider, or emic, point of view, as well as an outsider's, or etic, point of view of CRTI's value and utility (Morris, Leung, Ames, \& Lickel, 1999).

If instances of CRTI were to be identified along a continuum from low to high integration, then comparative studies could be done in matched samples (e.g., minority training performance in high, low, and no CRTI organizations) and correlative studies could evaluate the relationship between culturally responsive levels and learning and performance. Beyond correlational studies, researchers should experimentally manipulate the degree of cultural responsiveness to identify the critical dosage CRTI and potential for plateau. With regard to the dependent variable, research should be conducted where outcome conceptualization and measurement occasions are more distal, behaviorallybased, and considers the value added (e.g., ROI, employee retention).

In line with Baldwin and Ford's (1988) model of training, research should also look beyond the training design to the larger organizational context of training. Researchers might consider how impressions of the organization and its management are negatively affected when CRTI is unsupported by the general structure and culture of the organization. CRTI might work to set up false expectations from the trainees and make 
the organization appear deceptive. Ideally, an organization would support the principles incorporated into their training to the fullest extent possible. However, to preemptively buffer against the threat of expectation-experience discrepancies, the use of CRTI would need to be stated explicitly and couched within the mission statement of the organization, perhaps as a way to appreciate employees and provide an organizational culture that seeks to positively respond to diversity. The claim that unsupported CRTI post-training causes negative perceptions or that negative perceptions can be buffered by an explanation require empirical support. With each study researchers should be careful to list the components of their design, training objectives, and effect sizes for outcomes of interest so that meta-analyses can be run to control for method and context effects and moderators, and the true relationship between culturally responsive designs and performance can be assessed.

Trainee characteristics. My sample included a multi-national group, considering multiple tribes sovereign nations. However, given that North American tribes all share the experience of colonization, similar institutional struggles, and a collective group identity (i.e., Native American), suggestions for design and delivery that are applied to more or less homogenous groups should be implemented with caution. Future research should delineate the scope of CRTI, whether broad or specific, to more or less diverse cultural settings. Perhaps in certain settings, advocating for an emphasis on organizational culture trumps CRTI decisions. This may be the case when members of a cultural group do not identify or fit into their cultural group's norms or socialization 
experiences (e.g., $4^{\text {th }}$ generation versus $1^{\text {st }}$ generation immigrants in an ethnic neighborhood).

In this study, the instructor was a mixed heritage Native American doctoral student. CRTI should be tested with both Native and non-Native instructors with varying levels of expertise. Depending on the objective of the training and organization context, employees may be resistant to trust or comply with an out-group member more than an in-group member (e.g., for industries near border towns). For marginalized groups, internalized stigma (i.e., self-stereotyping) could have an opposite effect and in-group members may be deemed less trustworthy. Similarly, the level of education of the instructor may influence perceptions of credibility and participant engagement.

Finally, future research should explore the relation of CRTI to different forms of cognitive processing. It is possible that improved performance takes place due to the availability of more neural and associative networks. However, it is also possible that post-training performance does not improve or goes down because participants feel more comfortable, more focused on contemplation, and less concerned about memorization or self-presentation. Kulik and Roberson (2008) found that non-whites were more likely to transfer lessons from training than Whites. Perhaps the added cognitive difficulty of having to translate information cross-culturally (i.e., in terms of associate networks) facilitates greater processing. In that case our goal for training would be to create a culturally incongruent experience for all trainees to heighten cognitive processing.

Despite its rich historical foundation, culturally responsive approaches are understudied, especially in adult education. This study helped to fill this gap in at least 
Responsive Training 126

three areas. First, it imported the concept and rationale of culturally responsive approaches to the field of training. Second, it advanced the education literature on CRE in terms of its methodologically rigorous design. Third, this study helped Native American educators develop instructional strategies that have empirical backing. 


\section{References}

ACT Inc. (2013). ACT profile report - State: Oregon. Retrieved April 13, 2014 from https://www.act.org/newsroom/data/2013/pdf/profile/Oregon.pdf.

Agbo, S. (2004). First Nations perspectives on transforming the status of culture and language in schooling. Journal of American Indian Education, 43, 1-31.

Aguilera, D., Lipka, J., Demmert, W., \& Tippeconnic, J. (2007). Special issue on culturally responsive education for American Indian, Alaska Native, and native Hawaiian students. Journal American Indian Education, 46, 4-10.

Aguinis, H. \& Kraiger, K. (2009). Benefits of training and development for individuals, teams, organizations, and society. Annual Review of Psychology, 60, 451-474.

Aikenhead, G.S. \& Ogawa, M. (2007). Indigenous knowledge and science revisited. Cultural Studies of Science Education, 2, 539-620.

Alaskan Native Knowledge Network [ANKN]. (1998). Alaska rural systemic initiative: Year three annual progress report, December 1, 1997-November 30, 1998. Fairbanks: University of Alaska.

Allen, N.J. \& Crawley, F.E. (1998). Voices from the bridge: Worldview conflicts of Kickapoo students of science. Journal of Research in Science Teaching, 35, 111-132.

Alliger, G. M. \& Janak, E. A. (1989). Kirkpatrick's levels of training criteria: Thirty years later. Personnel Psychology, 42, 331-342.

Alliger, G. M., Tannenbaum, S. I., Bennett, W., Traver, H., \& Shotland, A. (1997). A meta-analysis of the relations among training criteria. Personnel Psychology, 50, 341358. 
American Psychological Association (APA). (2003). Guidelines on multicultural education, training, research, practice, and organizational change for psychologists. American Psychologist, 58, 377-402.

Anderson, J. R. (1996). ACT: A simple theory of complex cognition. American Psychologist, 51, 355.

Arthur Jr, W., Bennett Jr, W., Stanush, P. L. \& McNelly, T. L. (1998). Factors that influence skill decay and retention: A quantitative review and analysis. Human Performance, 11, 57-101.

Arthur, W. \& Bennett, W. (1995). The international assignee: The relative importance of factors perceived to contribute to success. Personnel Psychology, 48, 99-114.

Arthur, W., Bennett, W., Edens, P.S., \& Bell, S.T. (2003). Effectiveness of training in organizations: A meta-analysis of design and evaluation features. Journal of Applied Psychology, 88, 234-245.

Atkinson, T. (1769). Charter of Dartmouth College. Retrieved June 15, 2014 from http://www.dartmouth.edu/ trustees/docs/charter-2010.pdf.

ATSD (2013). State of the industry report (description). Retrieved March 20 ${ }^{\text {th }}, 2014$ from http://www.astd.org/Professional-Resources/State-Of-The-Industry-Report.

Augustine, N. R. (2005). Rising above the gathering storm: Energizing and employing America for a brighter economic future. Retrieved July, 20, 2014 from http://www.mdworkforce.com/pub/pdf/aaugustine10202005.pdf.

Averill, R., Anderson, D., Easton, H., Maro, P. T., Smith, D., \& Hynds, A. (2009). Culturally responsive teaching of mathematics: Three models from linked 
studies. Journal for Research in Mathematics Education, 157-186.

Bacon, H.L., Kidd, G., \& Seaberg, J. (1982). The effectiveness of bilingual instruction with Cherokee Indian students. Journal of American Indian Education, 21, 34-43.

Baldwin, T. T., \& Ford, J. K. (1988). Transfer of training: A review and directions for future research. Personnel Psychology, 41, 63-105.

Baldwin, T. T., \& Magjuka, R. J. (1997). Training as an organizational episode:

Pretraining influences on trainee motivation. Improving training effectiveness in work organizations, 99-127.

Ball, J. (2005). As if Indigenous knowledge and communities mattered: Transformative education in First Nations communities in Canada. American Indian Quarterly, 28, 454-479.

Balter, A. \& Grossman, F. (2009). The effects of the No Child Left Behind Act on the language and culture education in Navajo public schools. Journal of American Indian Education, 48, 19-46.

Banks, J. A. (2007). Educating citizens in a multicultural society ( $2^{\text {nd }}$ ed.). New York: Teachers College Press.

Banks, J. A. (2013). The Construction and Historical Development of Multicultural Education, 1962-2012. Theory Into Practice, 52, 73-82.

Banks, J. A., \& Banks, C. A. M. (Eds.). (2009). Multicultural education: Issues and perspectives. John Wiley \& Sons.

Banks, J. A., \& Tucker, M. (1998). Multiculturalism's five dimensions. NEA Today, 17, $17-20$. 
Barnhardt, C. (1994). Life on the other side: Alaska Native teacher education students and the University of Alaska Fairbanks. Doctoral dissertation, University of Alaska Fairbanks.

Barnhardt, R. \& Kawagley, A.O. (2005). Indigenous knowledge systems and Alaska native ways of knowing. Anthropology \& Education Quarterly, 36, 8-23.

Baron, R. M., \& Kenny, D. A. (1986). The moderator-mediator variable distinction in social psychological research: Conceptual, strategic, and statistical considerations. Journal of Personality and Social Psychology, 51, 1173.

Bartels, L.K., Nadler, J.T., Kufahl, K. and Pyatt, J. (2013). 'Fifty years after the Civil Rights Act: diversity management practices in the field'. Industrial and Organizational Psychology, 6, 450-457.

Battiste, M. (2002). Indigenous knowledge and pedagogy in First Nations education: A literature review with recommendations. Ottawa: Apamuwek Institute.

Bennett, R. (1987). Cooperative learning with a computer in a Native language class. Paper presented at the association of California State University Professors' Conference on the use of Personal Computers in Higher Education, San Diego, CA, March 26-27th.

Bennett, R., Aston, A., \& Colquhoun, T. (2000). Cross-cultural training: A critical step in ensuring the success of international assignments. Human Resource Management, 39, 239-250.

Berkes, F., Colding, J., \& Folke, C. (2000). Rediscovery of traditional ecological knowledge as adaptive management. Ecological applications, 10, 1251-1262. 
Berry, B. (1968). The Education of the American Indians: A Survey of the Literature. Office of Education, Bureau of Research: Washington DC.

Berry, J. W. (1966). Temne and Eskimo perceptual skills. Journal of International Psychology, 1, 207-299.

Berry, J. W. (1969). Ecology and socialization as factors in figural assimilation and the resolution of binocular rivalry. International Journal of Psychology, 4, 271-280.

Berry, J. W. (1971). Ecological and cultural factors in spatial perceptual development. Canada Journal of Behavioral Science, 3, 324-336.

Berry, J. W. (1967). Independence and conformity in subsistence-level societies. Journal of Personality and Social Psychology, 7, 415-418.

Bersin, J. (February 4th, 2014). Spending on corporate training soars: Employee capabilities now a priority. Retrieved February 10th, 2014 from http://www.forbes.com/sites/joshbersin/2014/02/04/the-recovery-arrives-corporatetraining-spend-skyrockets/.

Bezrukova, K., Jehn, K.A. \& Spell, C.S. (2012). Reviewing diversity training: Where we have been and where we should go. Academy of Management Learning \& Education, $11,207-227$.

Bhaskar-Shrinivas, P., Harrison, D. A., Shaffer, M. A., \& Luk, D. M. (2005). Input-based and time-based models of international adjustment: Meta-analytic evidence and theoretical extensions. Academy of Management Journal, 48, 257-281.

Bieschke, K. J., Bishop, R. M., \& Garcia, V. L. (1996). The utility of the research selfefficacy scale. Journal of Career Assessment, 4, 59-75. 
Black, J. S. \& Mendenhall, M. (1990). Cross-cultural training effectiveness: A review and a theoretical framework for future research. Academy of Management Review, 15, 113-136.

Bloom, B. S., Davis, A., Hess, R. D., \& Silverman, S. B. (1965). Compensatory education for cultural deprivation. New York: Holt, Rinehart and Winston.

Blume, B. D., Ford, J. K., Baldwin, T. T., \& Huang, J. L. (2010). Transfer of training: A meta-analytic review. Journal of Management, 36, 1065-1105.

Boudreau, J.W. \& Ramstad, P.M. (2005). Talentship, talent segmentation, and sustainability: A new HR decision science paradigm for a new strategy definition. Human Resource Management, 44, 129-136.

Brayboy, B. M. J. (2005). Toward a tribal critical race theory in education. The Urban Review, 37, 425-446.

Brayboy, B. M. J., \& Castagno, A. E. (2009). Self-determination through self-education: Culturally responsive schooling for Indigenous students in the USA.Teaching Education, 20, 31-53.

Brayboy, B.M.J. (2004). Hiding in the ivy: American Indian students and visibility in elite educational settings. Harvard Educational Review, 74, 125-152.

Brenner, M.E. (1998). Adding cognition to the formula for culturally relevant instruction in mathematics. Anthropology \& Education Quarterly, 29, 214-244.

Brewer, A. \& Suchan, T.A. (2001). Mapping census 2000: The geography of U.S. diversity. Redlands, CA: ESRI Press.

Brislin, R. W. \& Pedersen, P. (1976) Cross-cultural orientation programs. New York: 
Gardner Press.

Brook, D. (1998). Environmental genocide: Native Americans and Toxic Waste. American Journal of Economics and Sociology, 57, 105-113.

Brown, K.G. \& Sitzmann, T. (2011). Training and employee development for improved performance. In Zedeck, S. (Ed.), APA handbook of industrial and organizational psychology, Vol 2: Selecting and developing members for the organization. APA Handbooks in Psychology (pp. 469-503). Washington, DC, US: American Psychological Association.

Brown-Jeffy, S., \& Cooper, J. E. (2011). Toward a Conceptual Framework of Culturally Relevant Pedagogy: An Overview of the Conceptual and Theoretical

Literature. Teacher

Education Quarterly, 38, 65-84.

Bureau of Indian Affairs [BIA] (2014). Frequently asked questions. Retrieved on May 28, 2014, from http://www.bia.gov/FAQs/index.htm.

Burke, L. A. \& Hutchins, H. M. (2007). Training transfer: An integrative literature review. Human Resource Development Review, 6, 263-296.

Burke, L. A. \& Hutchins, H. M. (2008). A study of best practices in training transfer and proposed model of transfer. Human Resource Development Quarterly, 19, 107-128.

Busch, H. (2005). Is science education relevant?. Europhysics News, 36, 162-167.

Bushnell, D. S. (1990). Input, process, output: A model for evaluating training. Training and Development Journal, 44, 41-43.

Cajete, G. (1994). Look to the mountain: An ecology of indigenous education. Kivaki 
Press, 585 E. 31 st St., Durango, CO 81301.

Cajete, G. A. (1999). The Native American Learner and Bicultural Science Education. In K.C. Swisher and J.W. Tippeconnic III (Eds.), Next Steps: Research and Practice to Advance Indian Education. Charleston, WV: ERIC Clearinghou se on Rural Education and Small Schools.

Cajete, G.A. (2000). Native science: Natural laws of interdependence. Santa Fe, NM: Clear Light Publishers.

Callahan, J. S., Kiker, D. S., \& Cross, T. (2003). Does method matter? A meta-analysis of the effects of training method on older learner training performance. Journal of Management, 29, 663-680.

Campbell, J.P. (1971). Personnel training and development. Annual Review of Psychology, 22, 565-602.

Cannon, M. D., \& Witherspoon, R. (2005). Actionable feedback: Unlocking the power of learning and performance improvement. The Academy of Management Executive, 19, 120-134.

Cannon-Bowers, J. A., Rhodenizer, L., Salas, E., \& Bowers, C. A. (1998). A framework for understanding pre-practice conditions and their impact on learning. Personnel Psychology, 51, 291-320.

Cannon-Bowers, J. A., Tannenbaum, S. I., Salas, E., \& Converse, S. A. (1991). Toward an integration of training theory and technique. Human Factors, 33, 281-292.

Carter, M. \& Beier, M. E. (2010). The effectiveness of error management training with working-aged adults. Personnel Psychology, 63, 641-675. 
Cascio, W.F. \& Aguinis, H. (2011). Applied psychology in human resource management (7th ed.). Upper saddle River, NJ: Pearson Prentice Hall.

Castagno, A.E. \& Brayboy, B.M.J. (2008). Cultural responsive schooling for Indigenous youth: A review of the literature. Review of Educational Research, 78(4), 941-993.

Chambers, D. W. \& Gillespie, R. (2000). Locality in the history of science: Colonial science, technoscience, and indigenous knowledge. Osiris, 221-240.

Chapman, C., Laird, J., Ifill, N., \& KewalRamani, A. (2011). Trends in high school dropout and completion rates in the United States: 1972-2009 compendium report. Washington, DC: National Center for Education Statistics.

Chrobot-Mason, D., \& Quiñones, M. A. (2002). Training for a diverse workplace. In K. Kraiger (Ed.), Creating, implementing and managing effective training and development, 117-159. San Fransico, CA: Jossey-Bass.

Clark, W.J. (1996). Effects of a computer assisted instruction program on Aboriginal student achievement. Unpublished master's thesis, Brandon University, Manitoba, Canada. (ERIC Document Reproduction Service No. ED 400777)

Cleary, L. M., \& Peacock, T. D. (1998). Collected wisdom: American Indian education. Allyn \& Bacon, 160 Gould Street, Needham Heights, MA 02194.

Clinton, W. (1998). Executive Order 13096 of August 6, 1998: American Indian and Alaskan Native Education. Washington DC: The Federal Register, 63(104), 4268242684.

Cohen, F. S. (1945). Handbook of Federal Indian Law: With Reference Tables and Index. Washington, DC: US Government Printing Office. 
Cohen, J. (1988). Statistical power analysis for the behavioral sciences. Hillsdale, NJ: Erlbaum.

Colquitt, J. A., \& Simmering, M. J. (1998). Conscientiousness, goal orientation, and motivation to learn during the learning process: A longitudinal study. Journal of Applied Psychology, 83, 654.

Colquitt, J. A., LePine, J. A., \& Noe, R. A. (2000). Toward an integrative theory of training motivation: a meta-analytic path analysis of 20 years of research. Journal of Applied Psychology, 85, 678.

Colquitt, J. A., Scott, B. A., Rodell, J. B., Long, D. M., Zapata, C. P., Conlon, D. E., \& Wesson, M. J. (2013). Justice at the millennium, a decade later: a meta-analytic test of social exchange and affect-based perspectives. Journal of Applied Psychology, 98, 199.

Cottrell, M.C. (1971). Bilingual education in San Juan County, Utah: A cross cultural emphasis. Paper presented at the annual meeting of the American Educational Research Association, New York, NY. (ERIC Document Reproduction Service No. ED047855)

Cozby, P.C. \& Bates, S.C. (2012). Methods in behavioral research (11th ed). New York, NY: McGraw Hill.

Cronbach, L. J. \& Snow, R. E. (1977). Aptitudes and instructional methods: A handbook for research on interactions. Oxford, England: Irvington.

Curry, L. (1987). Integrating concepts of cognitive or learning styles: A review with attention to psychometric standards. Ottawa, Canada: Canadian College of Health 
Service Executives.

Curry-Stevens, A., Cross-Hemmer, A., \& Coalition of Communities of Color. (2011).

The Native American community of Multnomah county: An unsettling profile.

Portland, OR: Portland State University.

DeJong, D. H. (1993). Promises of the Past: A History of Indian Education in the United States. Golden, CO: North American Press.

Demmert, W. G., Jr., \& Towner, J. C. (2003). A review of the research literature on the influences of culturally based education on the academic performance of Native American student. Portland, OR. Northwest Regional Educational Laboratory (http://www.nwrel.org/indianed/cbe.pdf).

Demmert, W.G. (2001). Improving academic performance among Native American students: A review of the research literature. Charleston, WV: ERIC Clearinghouse on Rural Education and Small Schools, Appalachian Educational Laboratory.

Demmert, W.G., McCardle, P, Mele-McCarthy, J., \& Leos, K. (2006). Preparing Native American children for academic success: A blueprint for research. Journal of American Indian Education, 45, 92-106.

DeNavas-Walt, C. \& Proctor, B.D. (2014). Income and poverty in the United States: 2013, current population reports. Washington, D.C.: U.S. Government Printing Office.

Department of Education [DOE]. (2011). 30th Annual Report to Congress on the Implementation of the Individuals with Disabilities Education Act, 2008. Washington, D.C: Office of Special Education and Rehabilitative Services, Office of Special 
Education Programs.

Department of Labor, US [DOL]. (2011). Labor force chracteristics by race and ethnicity, 2010. Washington, DC: U.S. Bureau of Labor Statistics.

DeRue, D. S., Nahrgang, J. D., Wellman, N. E. D., \& Humphrey, S. E. (2011). Trait and behavioral theories of leadership: An integration and meta-analytic test of their relative validity. Personnel Psychology, 64, 7-52.

Deshpande, S. P. \& Viswesvaran, C. (1992). Is cross-cultural training of expatriate managers effective: A meta analysis. International Journal of Intercultural Relations, 16, 295-310.

Deyhle, D., \& Swisher, K. (1997). Research in American Indian and Alaska Native education: From assimilation to self-determination. Review of Research in Education, 22, 113-194.

Dunn, R. \& Dunn, K. (1992). Teaching elementary students through their individual learning styles. Boston: Allyn \& Bacon.

Dunn, R., Beaudry, J. S., \& Klavas, A. (2002). Survey of research on learning styles. California Journal of Science Education, 2, 75-98.

Ehrke, F., Berthold, A., \& Steffens, M.C. (2014). How diversity training can change attitudes: Increasing perceived complexity of superordinate groups to improve intergroup relations. Journal of Experimental Social Psychology, 53, 193-206.

Energy Information Administration. (2000). Energy consumption and renewable energy development potential on Indian Lands. U.S. Department of Energy: Washington, D.C. 
Ericsson, K. A., Krampe, R. T., \& Tesch-Römer, C. (1993). The role of deliberate practice in the acquisition of expert performance. Psychological Review, 100, 363.

Esen, E. (2005). 2005 Workplace Diversity Practices: Survey Report. Alexandria, VA: Society for Human Resource Management.

Felder, R. M., \& Spurlin, J. (2005). Applications, reliability and validity of the index of learning styles. International Journal of Engineering Education, 21, 103-112.

Ferraro, G. (2001). Cultural anthropology: An applied perspective (4 ${ }^{\text {th }}$ ed.). Belmont, CA: Wadsworth.

Fidell, L.S. \& Tabachnick, B.G. (2003). In Schinka, J.A. \& Velicer, W.F. (Eds.), "Preparatory data analysis". In Handbook of psychology: Research methods in psychology, Vol. 2, 115-141. New York, NY: John Wiley Sons.

Fiedler, F. E., Mitchell, T., \& Triandis, H. C. (1971). The culture assimilator: An approach to cross-cultural training. Journal of Applied Psychology, 55, 95.

Fleming, W. C. (2006). Myths and stereotypes about Native Americans. Phi Delta Kappan, 88, 213.

Franks, M.E. (1988). Using the gap reduction model to evaluate a successful bilingual/ESL program. Paper presented at the annual meeting of the Mid-South Educational Research Association, Louisville, KY. (ERIC Document Reproduction Service No. ED303505)

Fusco, D. (2001). Creating relevant science through urban planning and gardening. Journal of Research in Science Teaching, 38, 860-877.

Galliher, R. V., Jones, M. D., \& Dahl, A. (2011). Concurrent and longitudinal effects of 
ethnic identity and experiences of discrimination on psychosocial adjustment of Navajo adolescents. Developmental Psychology, 47, 509.

Gary, F. (2007). Cultural Anthropology an Applied Perspective (7th ed.). Belmont, CA: Wadsworth.

Gay, G. (1983). Multiethnic education: Historical developments and future prospects. Phi Delta Kappan, 560-563.

Gay, G. (2000). Culturally responsive teaching: Theory, research, and practice. New York: Teachers College Press.

Gay, G. (2002). Preparing for culturally responsive teaching. Journal of Teacher Education, 53, 106-116.

Gilliland, H. (1995). Teaching the Native American ( $3^{\text {rd }}$ ed.). Kendal/Hunt: Dubuque, IA. Goldstein, I.L. \& Ford, J.K. (2002). Training in Organizations (4th ed.). Belmont, CA: Wadsworth.

Goldstein, I.L. (1980). Training in work organizations. Annual Review of Psychology, 31, $229-272$.

Goldstein, I.L. (1986). Training in organizations (2nd ed.). Pacific Grove, CA: Brooks/Cole.

Greymorning, S. (1997). Going beyond words: The Arapaho immersion program. In J. Reyhner (Ed.), Teaching indigenous languages (pp. 22-30). Flagstaff, AZ: Northern Arizona University (ERIC Document Reproduction Service No. ED415061)

Grossman, R. \& Salas, E. (2011). The transfer of training: What really matters. International Journal of Training and Development, 15, 103-120. 
Gryczynki, J., Feldman, R., Carter-Pokras, O., Kanamori, M., Chen, L. \& Roth, S. (2010). Contexts of tobacco use and perspectives on smoking cessation among a sample of urban American Indians. Journal of Health Care for the Poor and Underserved, 21, 544-558.

Havighurst, Robert J. (1970). The education of Indian children and youth. The national study of American Indian education, Summary report and recommendations. USCE OEO-0-8-080147-2805, Series IV, No. 6. Chicago, IL: University of Chicago Press. Herman, A.M. (1999). Futurework: The trends and challenges of the 21st century. The United States Department of Labor: Washington, D.C.

Hickling-Hudson, A. R. (2003). Multicultural education and the postcolonial turn. Policy Futures in Education, 1, 381-401.

Hickling-Hudson, A. R. (2005). 'White','Ethnic'and 'Indigenous'. Pre-service teachers reflect on discourses of ethnicity in Australian culture. Policy Futures in Education, 3, $340-358$.

Hickling-Hudson, A. R. \& Alquist, R. (2003). Contesting the curriculum in the schooling of Indigenous children in Australia and the United States: From Eurocentrism to culturally powerful pedagogies. Comparative Education Review, 47, 64-89.

Hilberg, R. S., \& G Tharp, R. (2002). Theoretical perspectives, research findings, and classroom implications of the learning styles of American Indian and Alaska Native students. Center for Research on Education, Diversity \& Excellence.

Hill, S.T. (2007). Science and engineering degrees, by racelethnicity of recipients: 1995 2004. (NSF 07-308). Arlington VA: National Science Foundation, Division of Science 
Responsive Training 142

Resources Statistics (DRS).

Hobbs, F. \& Stoops, N. (2002). Demographic Trends in the Twentieth Century. Census 2000 Special Reports. Washington, DC: US Government Printing Office.

Hobbs, F., \& Stoops, N. (2002). Demographic trends in the 20th century (Vol. 4). US Census Bureau. Chicago.

Hofstede, G. (1980). Culture and organizations. International Studies of Management \& Organization, 15-41.

Hofstede, G. (1998). Attitudes, values and organizational culture: Disentangling the concepts. Organization Studies, 19, 477-493.

Hodge, F.S. (2012). No meaningful apology for American Indian unethical research abuses. Ethics \& Behavior, 22, 431-444.

Hollenbeck, J. R., Williams, C. L., \& Klein, H. J. (1989). An empirical examination of the antecedents of commitment to difficult goals. Journal of Applied Psychology, 74, $18-23$.

Holton III, E. F., \& Baldwin, T. T. (2003). Improving learning transfer in organizations. San Fransico, CA: Jossey-Bass.

Holton III, E. F., Bates, R. A., \& Ruona, W. E. (2000). Development of a generalized learning transfer system inventory. Human Resource Development Quarterly, 11, 333360.

Houghton, N. D. (1931). The legal status of Indian suffrage in the United States. California Law Review, 507-520.

Howard, K. W. (1989). A comprehensive expectancy motivation model: Implications for 
adult education and training. Adult Education Quarterly, 39, 199-210.

Howard, T. C. (2003). Culturally relevant pedagogy: Ingredients for critical teacher reflection. Theory into Practice, 42, 195-202.

Howell, W.C. \& Cooke, N.J. (1989). Training in human information processor. In I.L. Goldstein (Ed.), Training and development in organizations (pp. 121-182). San Fransico, CA: Jossey-Bass.

Hu, L. \& Bentler, P.M. (1999). Cutoff criteria for fit indexes in covariance structure analysis: Conventional criteria versus new alternatives. Structural Equation Modeling: A Multidisciplinary Journal, 6, 1-55.

Hunt, D. (January 30th, 2012). BIA's impact on Indian education is an education in bad eduation. Indian Country Today Media Network.

Indian Nations at Risk Task Force (1991). Indian nations at risk: An educational strategy for action. Washington, DC: US Department of Education.

Ingalls, L. \& Hammond, H. (2007). The match between Apache Indians' culture and educational practices used in our schools: From problems to solutions. College Teaching Methods \& Styles Journal (CTMS), 3, 9-18.

Jackson, S. E., Joshi, A., \& Erhardt, N. L. (2003). Recent research on team and organizational diversity: SWOT analysis and implications. Journal of Management, 29, 801-830.

Jackson, S.E. \& Joshi, A. (2011) Work team diversity. In: Zedeck S (ed.) APA Handbook of Industrial and Organizational Psychology, Vol. 1. Washington, DC:American Psychological Association Press, 651-686. 
Jahoda, G. (2012). Critical reflections on some recent definitions of "culture".Culture \& Psychology, 18, 289-303.

James, K. (2000). Social psychology: American Indians, science and technology. Social Science Computer Review, 18, 196-213.

James, K. (2001). Science and Native American communities: Legacies of pain, visions of promise. Lincoln and London: University of Nebraska Press.

James, K. (2006). Identity, cultural values, and American Indians' perceptions of science and technology. American Indian Culture and Research Journal, 30, 45-58.

James, K., \& Cardador, J. (2007). Cognitions about technology and science: A measure and its relevance to career decisions. Journal of Career Assessment, 15, 463-482.

James, K., Chavez, E., Beauvais, F., Edwards, R., \& Oetting, G. (1995). School achievement and dropout among Anglo and Indian females and males: A comparative examination. American Indian Culture and Research Journal, 19, 181-206.

James, K., Hall, D., Redsteer, M.H., \& Doppelt, R. (2008). Organizational environmental justice with a Navajo (Dine) nation case example. In S. Gilliliand, D.D. Steiner, and D.P. Skarlicki, Justice, morality, and social responsibility (pp. 263-289). Charlott, NC: Information Age Publishing.

Jaramillo, F., Mulki, J. P., \& Marshall, G. W. (2005). A meta-analysis of the relationship between organizational commitment and salesperson job performance: 25 years of research. Journal of Business Research, 58, 705-714.

Jensen, A. R. (1969). How much can we boost IQ and scholastic achievement. Harvard Educational Review, 39, 1-123. 
Johnston, W. B. (1991). Global workforce 2000: The new world labor market. Harvard Business Review, 69, 115-127.

Jones, K. P., King, E. B., Nelson, J., Geller, D. S., \& Bowes-Sperry, L. (2013). Beyond the business case: an ethical perspective of diversity training. Human Resource Management, 52, 55-74.

Kalinoski, Z.T., Steele-Johnson, D., Peyton, E.J., Leas, K.A., Steinke, J. \& Bowling, N.A. (2013). A meta-analytic evaluation of diversity training outcomes. Journal of Organizational Behavior, 34, 1076-1104.

Karl, K. A., O'Leary-Kelly, A. M., \& Martocchio, J. J. (1993). The impact of feedback and self-efficacy on performance in training. Journal of Organizational Behavior, 14, $379-394$.

Kawagley, A. O. \& Barnhardt, R. (1998). Education Indigenous to Place: Western Science Meets Native Reality. Fairbanks, AK: Alaska Native Knowledge Network.

Kealey, D. J. \& Protheroe, D. R. (1996). The effectiveness of cross-cultural training for expatriates: An assessment of the literature on the issue.International Journal of Intercultural Relations, 20, 141-165.

Keefe, J. W. (1979). Learning style: An overview. Student Learning Styles: Diagnosing and Prescribing Programs, 1-17.

Keith, N., \& Frese, M. (2008). Effectiveness of error management training: a meta analysis. Journal of Applied Psychology, 93, 59.

Kennedy, E. (1969). Indian Education: A National Tragedy-A National Challenge. Washington, DC: U.S. Government Printing Office. 
Kerbo, H. R. (1980). College achievement among Native Americans: A research note. Social Forces, 59, 1275.

Kierein, N. M., \& Gold, M. A. (2000). Pygmalion in work organizations: a meta-analysis. Journal of Organizational Behavior, 21, 913-928.

King, E.G., Dawson, J.F., Kravitz, D.A., \& Gulick, L.M.V. (2012). A multilevel study of the relationships between diversity training, ethnic discrimination and satisfaction in organizations. Journal of Organizational Behavior, 33, 5-20.

Kirkpatrick, D.L. (1976). Evaluation of training. In R.L. Craig (Ed.), Training and development handbook: A guide to human resource development (3rd ed., pp. 301319). New York: McGraw-Hill.

Klein, H. J., Wesson, M. J., Hollenbeck, J. R., Wright, P. M., \& DeShon, R. P. (2001). The assessment of goal commitment: A measurement model meta-analysis. Organizational Behavior and Human Decision Processes, 85, 32-55.

Kleinfeld, J., \& Nelson, P. (1988). Adapting Instruction to Native Americans'" Learning Styles": An Iconoclastic View. Fairbanks, AK: ERIC.

Klug, B. \& Whitfield, P. (2003). Widening the circle: Culturally relevant pedagogy for American Indian students. New York: Routledge.

Klump, J. \& McNeir, G. (2005). Culturally responsive practices for student success: A regional sampler. Northwest Regional Educational Laboratory: Portland, OR.

Kolb, D. A. (1981). Learning styles and disciplinary differences. The Modern American College, 232-255.

Kraiger, K. (2002a). Preface. In K. Kraiger (Ed.), Creating, implementing, and managing 
effective training and development. San Fransico, CA: Jossey-Bass.

Kraiger, K. (2002b). Decision-based evaluation. In K. Kraiger (Ed.), Creating, implementing, and managing effective training and development (pp. 331-375). San Fransico, CA: Jossey-Bass.

Kraiger, K., Ford, J.K., \& Salas, E. (1993). Application of cognitive, skill-based, and affective theories of learning outcomes to new models of training evaluation. Journal of Applied Psychology, 78, 311-328.

Kratochwill, T.R., McDonald, L., Young Bear-Tibbitts, H., \& Levin, J.R. (2001). Families and schools together: An experimental analysis of a parent-mediated early intervention program for at-risk American Indian children. Madison, WI: Wisconsin Center for Education Research.

Kulik, C. T. (2014). Working below and above the line: the research-practice gap in diversity management. Human Resource Management Journal, 24, 129-144.

Kulik, C. T., \& Roberson, L. (2008). Diversity initiative effectiveness: What organizations can (and cannot) expect from diversity recruitment, diversity training, and formal mentoring programs. In A. P. Brief (Ed.), Diversity at work (pp. 265-317). Cambridge: Cambridge University Press.

Ladson-Billings, G. (1989). A Tale of Two Teachers: Exemplars of Successful Pedagogy for Black Students. Paper presented at the Educational Equality Project Colloquium (New York, NY, May 4-5, 1989). Washington, DC: National Academy of Education. Ladson-Billings, G. (1990). Like lightning in a bottle: attempting to capture the pedagogical excellence of successful teachers of black students 1. Internation Journal 
of Qualitative Studies in Education, 3, 335-344.

Ladson-Billings, G. (1994). The dreamkeepers: Success-ful teaching for AfricanAmerican students. San Francisco: Jossey-Bass.

Ladson-Billings, G. (1995a). But that's just good teaching! The case for culturally relevant pedagogy. Theory into Practice, 34, 159-165.

Ladson-Billings, G. (1995b). Toward a theory of culturally relevant pedagogy. American Educational Research Journal, 32, 465-491.

LaFrance, J. (2004). Culturally competent evaluation in Indian country. New Directions for Evaluation, 102, 39-50.

LaFromboise, T. D., Trimble, J. E., \& Mohatt, G. V. (1990). Counseling Intervention and American Indian Tradition An Integrative Approach. The Counseling Psychologist, $18,628-654$.

Latham, G.P. (1988). Human resource training and development. Annual Review of Psychology, 39, 545-582.

Lee, T. S., \& Quijada Cerecer, P. D. (2010). (Re) Claiming Native Youth Knowledge: Engaging in Socio-culturally Responsive Teaching and Relationships. Multicultural Perspectives, 12, 199-205.

Lemke, J. L. (2001). Articulating communities: Sociocultural perspectives on science education. Journal of research in science teaching, 38, 296-316.

Lewis, D.R. (1995). Native Americans and the environment: A survey of twentiethcentury issues. American Indian Quarterly, 19, 423-450.

Lewis, J.L., Menzies, H., Najera, E.I., \& Page, R.N. (2009). Rethinking trends in 
minority participation in the sciences. Science Education, 93, 961-977.

Lievens, F., Harris, M. M., Van Keer, E., \& Bisqueret, C. (2003). Predicting crosscultural training performance: the validity of personality, cognitive ability, and dimensions measured by an assessment center and a behavior description interview. Journal of Applied Psychology, 88, 476.

Lipka, J., \& Adams, B. (2002). Improving Alaska Native rural and urban students' mathematical understanding of perimeter and area. Unpublished manuscript. Alaska School Research Fund.

Lipka, J., \& McCarty, T. L. (1994). Changing the culture of schooling: Navajo and Yup'ik cases. Anthropology \& Education Quarterly, 25, 266-284.

Littrel, L.N., Salas, E., Hess, K.P., Paley, M. \& Riedel, S. (2006). Expatriate preparation: A critical analysis of 25 years of cross-cultural training research. Human Resource Development Review, 5, 355-388.

Locke, E. A., \& Latham, G. P. (1990). A theory of goal setting \& task performance. Prentice-Hall, Inc.

Locke, E. A., \& Latham, G. P. (2002). Building a practically useful theory of goal setting and task motivation: A 35-year odyssey. American psychologist, 57, 705.

Lomawaima, K. T. (1999). The un-natural history of American Indian education. In K.G. Swisher \& J.W. Tippeconnic (Eds.) Next steps: Resarch and practice to advance Indian education (pp. 2-30). Charleston, WV: ERIC Clearinghouse on Rural Education and Small Schools.

Lomawaima, K. T., \& McCarty, T. L. (2002). When tribal sovereignty challenges 
Responsive Training 150

democracy: American Indian education and the democratic ideal. American Educational Research Journal, 39, 279-305.

López, F. A., Heilig, J. V., Schram, J. (2013). A Story within a Story: Culturally Responsive Schooling and American Indian and Alaska Native Achievement in the National Indian Education Study. American Journal of Education, 119, 513-538.

Lord, F. M. (1952). The relationship of the reliability of multiple-choice test to the distribution of item difficulties. Psychometrika, 18, 181-194.

Mack, E., Augare, H., Cloud-Jones, L. D., Davíd, D., Gaddie, H. Q., Honey, R. E., ... \& Wippert, R. (2012). Effective practices for creating transformative informal science education programs grounded in Native ways of knowing. Cultural Studies of Science Education, 7, 49-70.

Madera, J.M., King, E.B., \& Hebl, M.R. (2013). Enhancing the effects of sexual orientation diversity training: The effects of setting goals and training mentors on attitudes and behaviors. Journal of Business and Psychology, 28, 79-91.

Maina, F. (1997). Culturally relevant pedagogy: First Nations education in Canada. The Canadian Journal of Native Studies, 17, 293-314.

Major, D.A., Turner, J.E., \& Fletcher, T.D. (2006). Linking proactive personality and the big five to motivation to learn and development activity. Journal of Applied Psychology, 91, 927-935.

McCarty, T. L., Lynch, R. H., Wallace, S. \& Benally, A. (1991). Classroom inquiry and Navajo learning styles: A call for reassessment. Anthropology \& Education Quarterly, 22, 42-59. 
McGehee, W. \& Thayer, P.W. (1961). Training in business and industry. Oxford, England: Wiley.

Merriam, L. (1928). The Problem of Indian Administration: Report of a Survey Made at the Request of Honorable Hubert Work, Secretary of the Interior. Baltimore: The Institute for Government Research.

Meyer, J. P., Allen, N. J., \& Smith, C. A. (1993). Commitment to organizations and occupations: Extension and test of a three-component conceptualization. Journal of Applied Psychology, 78, 538.

Meyer, J. P., Stanley, D. J., Herscovitch, L., \& Topolnytsky, L. (2002). Affective, continuance, and normative commitment to the organization: A meta-analysis of antecedents, correlates, and consequences. Journal of Vocational Behavior, 61, 20-52.

Michalak, D. F. (1981). The Neglected Half of Training. Training and Development Journal, 35, 22-28

Miles, C. (January $\left.27^{\text {th }}, 2012\right)$. Oregon graduation rate continues to increase. Oregon Department of Education, News Release. Retrieved April 14 ${ }^{\text {th }}, 2014$ from http://www.ode.state.or.us/news/announcements/announcement.aspx?ID=7983\& TypeID=5.

Miller, J.D. (1998). The measurement of civic scientific literacy. Public Understanding of Science, 7, 203-223.

Miller, J. D. (2004). Public understanding of, and attitudes toward, scientific research: What we know and what we need to know. Public Understanding of Science, 13, 273 294. 
Miller, L. (Novermber 8th, 2012). ASTD 2012 state of the industry report: Organizations continue to invest in workplace learning. Retrieved January 29th, 2014 from http://www.astd.org/Publications/Magazines/TD/TD-Archive/2012/11/ASTD-2012State-of-the-Industry-Report.

Mohatt, G., \& Erickson, F. (1981). Cultural differences in teaching styles in an Odawa school: A sociolinguistic approach. In H. Trueba, G. Guthrie, \& K. Au (Eds.), Culture and the bilingual classroom: Studies in classroom ethnography (pp. 105-199). Rowley, MA: Newbury House.

Mohatt, N. V., Thompson, A. B., Thai, N. D., \& Tebes, J. K. (2014). Historical trauma as public narrative: A conceptual review of how history impacts present-day health. Social Science \& Medicine, 106, 128-136.

Moran, J. R., Fleming, C. M., Somervell, P., \& Manson, S. M. (1999). Measuring Bicultural Ethnic Identity among American Indian Adolescents A Factor Analytic Study. Journal of Adolescent Research, 14, 405-426.

More, A. J. (1984). Okanagan Nicola Indian quality of education study. Okanagan Indian Learning Institute.

More, A. J. (1987). Native Indian learning styles: A review for researchers and teachers. Journal of American Indian Education, 27, 17-29.

More, A. J. (1990). Learning Styles of Native Americans and Asians. Paper presented at the Annual Meeting of the American Psychological Association (98th, Boston, MA). Morris, M. A. \& Robie, C. (2001). A meta-analysis of the effects of cross-cultural 
Responsive Training 153

training on expatriate performance and adjustment. International Journal of Training and Development, 5, 112-125.

Morris, M. W., Leung, K., Ames, D., \& Lickel, B. (1999). Views from inside and outside: Integrating emic and etic insights about culture and justice judgment. Academy of Management Review, 24, 781-796.

Morrison, K. A., Robbins, H. H., \& Rose, D. G. (2008). Operationalizing culturally relevant pedagogy: A synthesis of classroom-based research. Equity \& Excellence in Education, 41, 433-452.

Mosby, I. (2013). Administering colonial science: nutrition research and human biomedical experimentation in Aboriginal communities and residential schools, 19421952. Histoire Sociale/Social History, 46, 145-172.

Murry, A. T., James, K., \& Drown, D. (2013). From pictures to numbers: Vision mapping and sustainability collaboration between Native American community members and mainstream scientists. American Indian Culture and Research Journal, 37, 1-24.

Murtagh, E.J. (1982). Creole and English as languages of instruction in bilingual education with Aboriginal Australians: Some research findings. International Journal of the Sociology of Language, 36, 15-33.

Nagel, J. (1994). Constructing ethnicity: Creating and recreating ethnic identity and culture. Social Problems, 152-176.

Nagel, J. (1995). American Indian ethnic renewal: Politics and the resurgence of identity. American Sociological Review, 947-965.

National Indian Education Association (NIEA). (n.d.) Statistics on Native students. 
Retrieved June $17^{\text {th }}, 2014$ from http://www.niea.org/Research/Statistics.aspx.

Nieto, S. (1999). The light in their eyes: Creating multicultural learning communities. New York, NY: Teachers College Press.

Noe, R. (1986). Trainee's attributes and attitudes: Neglected influences on training effectiveness. Personnel Psychology, 46, 125-147.

Noe, R. (2008). Employee training and development (4th ed.). Boston, MA: McGrawHill.

Noe, R. A., \& Schmitt, N. (1986). The influence of trainee attitudes on training effectiveness: Test of a model. Personnel Psychology, 39, 497-523.

Noe, R.A. \& Colquitt, J.A. (2002). Planning for training impact: Principles of training effectiveness. In In K. Kraiger (Ed.), Creating, implementing, and managing effective training and development (pp. 53-79). San Fransico, CA: Jossey-Bass.

Norris, T., Vines, P. L., \& Hoeffel, E. M. (2012). The American Indian and Alaska Native Population: 2010. US Department of Commerce, Economics and Statistics Administration, US Census Bureau.

Norton, I. M., \& Manson, S. M. (1996). Research in American Indian and Alaska Native communities: navigating the cultural universe of values and process.Journal of Consulting and Clinical Psychology, 64, 856.

Oetting, E. R., Swaim, R. C., \& Chiarella, M. C. (1998). Factor structure and invariance of the orthogonal cultural identification scale among American Indian and Mexican American youth. Hispanic Journal of Behavioral Sciences, 20, 131-154.

Oetting, E.R., \& Beauvais, F (1990-1991). Orthogonal cultural identification theory: The 
cultural identification of minority adolescents. International Journal of Addiction, 25, $655-685$.

Office of Elementary and Secondary Education (OESE). (2005). History of Indian Education. Retrieved June, 16th 2014 from http://www2.ed.gov/about/offices/list/oese/oie/history.html.

Ogbu, J. U. (1992a). Adaptation to minority status and impact on school success. Theory into Practice, 31, 287-295.

Ogbu, J. U. (1992b). Understanding cultural diversity and learning. Educational Researcher, 21, 5-14.

Ogbu, J. U. (1995). Cultural problems in minority education: Their interpretations and consequences_-Part one: Theoretical background. The Urban Review, 27, 189-205.

Ogbu, J. U., \& Simons, H. D. (1998). Voluntary and involuntary minorities: a cultural-ecological theory of school performance with some implications for education. Anthropology \& Education Quarterly, 29, 155-188.

Ogunwale, S.U. (2006). We the people: American Indians and Alaska Native in the United States. Census 2000 Special Reports. Retrieved on June, 2006 from http://www.census.gov/prod/2006pubs/censr-28.pdf.

Omizo, M. M., \& Omizo, S. A. (1989). Art activities to improve self-esteem among Native Hawaiian children. The Journal of Humanistic Education and Development, $27,167-176$.

Omizo, M. M., Omizo, S. A., \& Kitaoka, S. K. (1998). Guided affective and cognitive imagery to enhance self-esteem among Hawaiian children. Journal of Multicultural 
Counseling and Development, 26, 52-62.

Osborne, J. \& Collins, S. (2001). Pupils' views of the role and value of the science curriculum: A focus-group study. International Journal of Science Education, 23, 441-467.

Osborne, J., Simon, S., \& Collins, S. (2003). Attitudes towards science: A review of the literature and its implications. International Journal of Science Education, 25, 10491079.

Osman-Gani, A. M. \& Rockstuhl, T. (2009). Cross-cultural training, expatriate selfefficacy, and adjustments to overseas assignments: An empirical investigation of managers in Asia. International Journal of Intercultural Relations, 33, 277-290.

Pager, D. \& Shepherd, H. (2008). The sociology of discrimination: Racial discrimination in employment, housing, credit, and consumer markets. Annual Review of Sociology, 34, 181.

Palthe, J. (2004). The relative importance of antecedents to cross-cultural adjustment: Implications for managing a global workforce. International Journal of Intercultural Relations, 28, 37-59.

Paluck, E. L. (2006). Diversity training and intergroup contact: A call to action research. Journal of Social Issues, 62, 577-595.

Pashler, H., McDaniel, M., Rohrer, D., \& Bjork, R. (2008). Learning styles concepts and evidence. Psychological science in the public interest, 9, 105-119.

Pavkov, T.W., Leah, T., Fox, K.A., King, C.B. \& Cross, T.L. (2008). Tribal youth victimization and delinquency: Analysis of youth risk behavior surveillance survey 
data. Cultural Diversity and Ethnic minority Psychology, 16, 123-134.

Pewewardy, C. (1998). Fluff and feathers: Treatment of Americans in the literature and the classroom. Equity \& Excellence, 31, 69-76.

Pewewardy, C. (2002). Learning styles of American Indian/Alaskan Native students: A review of the literature and implications for practice. Journal of American Indian Education, 41, 23.

Pewewardy, C. (2012). Culturally responsive teaching for American Indian students. In J. Diller \& J. Moule (Eds.), Cultural Competence: A primer for educators. Belmont, CA: Thompson Wadsworth.

Pewewardy, C. \& Fitzpatrick, M. (2009). Working with American Indian students and families: Disabilities, issues, and interventions. Intervention in School and Clinic, 45, 91-98.

Pewewardy, C., \& Hammer, P. C. (2003). Culturally Responsive Teaching for American Indian Students. Charleston, WV: ERIC Digest.

Philips, S. U. (1976). Some sources of cultural variability in the regulation of talk. Language in Society, 5, 81-95.

Philips, S.U. (1972). Participant structures and communicative competence. In C. Cazden, V. John, \& D. Hymes (Eds.), The function of lanuage in the classroom (pp. 370-394). New York: Teachers College Press.

Powers, B., \& Rothwell, W. J. (2007). Instructor excellence: Mastering the delivery of training. John Wiley \& Sons.

Rayner, S., \& Riding, R. (1997). Towards a categorisation of cognitive styles and 
learning styles. Educational Psychology, 17, 5-27.

Ren, H., Shaffer, M. A., Harrison, D. A., Fu, C., \& Fodchuk, K. M. (2014). Reactive adjustment or proactive embedding? Multistudy, multiwave evidence for dual pathways to expatriate retention. Personnel Psychology, 67, 203-239.

Reyhner, J. (1993). American Indian language policy and school success. The Journal of Educational Issues of Language Minority Students, 12, 35-59.

Reynolds, D., Rahman, I., \& Bradetich, S. (2013). Hotel manager's perceptions of the value of diversity training: an empirical investigation. International Journal Contemporary Hospitality Management, 23, 426-446.

Riding, R., \& Cheema, I. (1991). Cognitive styles — an overview and integration. Educational psychology, 11, 193-215.

Riggs, E. M. (1998). Toward an understanding of the roles of scientific, traditional, and spiritual knowledge in our "demon-haunted world". American Indian Culture and Research Journal, 22, 213-226.

Riggs, E. M. (2004). Field-based education and Indigenous knowledge: Essential components of geoscience education for Native American communities. Science Education, 1-18.

Riggs, E. M., Robbins, E., \& Darner, R. (2007). Sharing the land: Attracting Native American students to the geosciences. Journal of Geoscience Education, 55, 478.

Robers, S., Kemp, J., \& Truman, J. (2013). Indicators of School Crime and Safety: 2012. NCES 2013-036/NCJ 241446. Washington, DC: National Center for Education Statistics. 
Responsive Training 159

Roberson, L., Kulik, C. T., \& Pepper, M. B. (2009). Individual and environmental factors influencing the use of transfer strategies after diversity training. Group \& Organization Management, 34, 67-89.

Roberson, L., Kulik, C.T., \& Pepper, M.B. (2003). Using needs assessment to resolve controversies in diversity training design. Group \& Organizational Management, 28, 148-174.

Roehrig, G. H., Dubosarsky, M., Mason, A., Carlson, S., \& Murphy, B. (2011). We look more, listen more, notice more: Impact of sustained professional development on head start teachers' inquiry-based and culturally-relevant science teaching practices. Journal of Science Education and Technology, 20, 566-578.

Rogers, C. A. \& Jaime, A. M. (2010). Listening to the community: Guidance from Native community members for emerging culturally responsive educators. Equity \& Excellence in Education, 43, 188-201.

Rollings, W. H. (2004). Citizenship and Suffrage: The Native American Struggle for Civil Rights in the American West, 1830-1965. Neveda Law Journal, 5, 126-140.

Rosenthal, R., \& Jacobson, L. (1968). Pygmalion in the classroom: teacher expectation and pupils' intellectual development. New York: Holt, Rinehart \& Winston.

Rosier, P., \& Holm, W. (1980). The Rock Point experience: A longitudinal study of a Navajo school program. Washington, DC: Center for Applied Linguistics. (ERIC Document Reproduction Service No. ED195363)

Ross, A. C. (1982). Brain Hemispheric Functions and the Native American. Journal of American Indian Education, 21, 2-5. 
Responsive Training 160

Ross, T., Kena, G., Rathbun, A., KewalRamani, A., Zhang, J., Kristapovich, P., and Manning, E. (2012). Higher Education: Gaps in Access and Persistence Study (NCES 2012-046). U.S. Department of Education, National Center for Education Statistics. Washington, DC: Government Printing Office.

Rossi, P. H., Lipsey, M. W., \& Freeman, H. E. (2003). Evaluation: A systematic approach. Thousand Oaks, CA: Sage publications.

Rossi, P.H., Lipsey, M.W., \& Freeman, H.E. (2004). Evaluation: A systematic approach ( $7^{\text {th }}$ Ed.). Sage: Thousand Oaks, CA.

Roth, W. M., \& Lee, Y. J. (2007). "Vygotsky’s neglected legacy”: Cultural-historical activity theory. Review of Educational Research, 77, 186-232.

Rouiller, J. Z. \& Goldstein, I. L. (1993). The relationship between organizational transfer climate and positive transfer of training. Human Resource Development Quarterly, 4, $377-390$.

Rudin, C. (1989). Omaha Language Preservation in the Macy, Nebraska Public School. Washington, DC: National Science Foundation.

Ruggs, E. N., Law, C., Cox, C. B., Roehling, M. V., Wiener, R. L., Hebl, M. R., \& Barron, L. (2013). Gone fishing: I-O psychologists' missed opportunities to understand marginalized employees' experiences with discrimination. Industrial and Organizational Psychology, 6, 39-60.

Sackett, P. R. \& Mullen, E. J. (1993). Beyond formal experimental design: Towards an expanded view of the training evaluation process. Personnel Psychology, 46, 613-627. Salas, E. \& Cannon-Bowers, J. A. (2001). The science of training: A decade of 
progress. Annual Review of Psychology, 52, 471-499.

Salas, Tannenbaum, Kraiger, \& Smith-Jentsch (2012). The science of training and development in Organizations: What matters in practice. Psychological Science in the Public Interest, 13, 74-101.

Santamaria, L. J. (2009). Culturally responsive differentiated instruction: Narrowing gaps between best pedagogical practices benefiting all learners. The Teachers College Record, 111, 214-247.

Santoro, N. (2007). 'Outsiders' and 'others':'different'teachers teaching in culturally diverse classrooms. Teachers and Teaching: theory and practice,13(1), 81-97.

Santoro, N., Reid, J. A., Crawford, L., \& Simpson, L. (2011). Teaching indigenous children: Listening to and learning from indigenous teachers. Australian Journal of Teacher Education, 36, 65-76.

Sasson, J. R. \& Austin, J. (2005). The effects of training, feedback, and participant involvement in behavioral safety observations on office ergonomic behavior. Journal of Organizational Behavior Management, 24, 1-30.

Savage, C., Hindle, R., Meyer, L. H., Hynds, A., Penetito, W., \& Sleeter, C. E. (2011). Culturally responsive pedagogies in the classroom: Indigenous student experiences across the curriculum. Asia-Pacific Journal of Teacher Education, 39, 183-198.

Saxton, R. (2014). Statewide report card: An annual report to the legislature on Oregon public schools 2013-2014. Salem, OR: Oregon Department of Education. Retrieved January 15th, 2015 from http://www.ode.state.or.us/data/annreportcard/rptcard2014.pdf. 
Schmeichel, M. (2012). Good Teaching? An examination of culturally relevant pedagogy as an equity practice. Journal of Curriculum Studies, 44, 211-231.

Schneider, B. (1987). The people make the place. Personnel Psychology, 40, 437-453.

Scriven, M. (2013) Key evaluation checklist (KEC). Retrieved October 14th, 2014 from http://michaelscriven.info/images/KEC_7.25.2013.pdf

Semken, S. (2005). Sense of place and place-based introductory geoscience teaching for American Indian and Alaska Native undergraduates. Journal of Geoscience Education, 53, 149-157.

Semken, S.C. \& Morgan, F. (1997). Navajo pedagogy and earth systems. Journal of Geoscience Education, 45, 109-112.

Seymour, E. (2000). Tracking the processes of change in US undergraduate education in science, mathematics, engineering, and technology. Science Education 86, 79-105.

Siebens, J., \& Julian, T. (2011). Native North American Languages Spoken at Home in the United States: 2006-2010. US Department of Commerce, Economics and Statistics Administration, US Census Bureau.

Simosi, M. \& Xenikou, A. (2010). The role of organizational culture in the relationship between leadership and organizational commitment: an empirical study in a Greek organization. The International Journal of Human Resource Management, 21, 15981616.

Simosi, M. (2012). The moderating role of self-efficacy in the organizational culturetraining transfer relationship. International Journal of Training and Development, 16, 92-106. 
Sleeter, C. E. (2011). The Academic and Social Value of Ethnic Studies: A Research Review. Washington, DC: National Education Association Research Department.

Smith-Jentsch, K. A., Jentsch, F. G., Payne, S. C., \& Salas, E. (1996). Can pretraining experiences explain individual differences in learning?. Journal of Applied Psychology, 81, 110.

Snow, R. E. (1986). Individual differences and the design of educational programs. American Psychologist, 41, 1029.

Soldier, L. L. (1988). Sociocultural Context and Language Learning of Native American Pupils. ERIC Clearinghouse.

Sperry, R. W. (1961). Cerebral organization and behavior. Science, 133(3466), 17491757.

Spindler, L., \& Spindler, G. (1958). Male and female adaptations in culture change. American Anthropologist, 60, 217-233.

Stahl, G. K. \& Caligiuri, P. (2005). The effectiveness of expatriate coping strategies: the moderating role of cultural distance, position level, and time on the international assignment. Journal of Applied Psychology, 90, 603.

Steele, C.M. \& Aronson, J. (1995). Stereotype threat and the intellectual test performance of African Americans. Journal of Personality and Social Psychology, 69, 797-811.

Sterenberg, G. (2013). Considering Indigenous Knowledges and Mathematics Curriculum. Canadian Journal of Science, Mathematics and Technology Education, 13, 18-32.

Stiles, D. B. (1997). Four Successful Indigenous Language Programs. In J.A. Reyhner 
(Ed.), Teaching indigenous languages (pp. 148-262). Northern Arizona University:

Center for Excellence in Education.

Südkamp, A., Kaiser, J., \& Möller, J. (2012). Accuracy of teachers' judgments of students' academic achievement: A meta-analysis. Journal of Educational Psychology, 104(3), 743.

Swanson, L.J. (1995). Learning styles: A review of the literature. Education Resources Information Center (ERIC).

Swisher, K. (1990). Cooperative learning and the education of American Indian/Alaskan Native students: A review of the literature and suggestions for implementation. Journal of American Indian Education, 29, 36-43.

Szasz, M. C. (1983). American Indian education: Historical perspective. Peabody Journal of Education, 61, 109-112.

Tabachnick, B.G. \& Fidell, L.S. (2007). Using multivariate statistics $\left(5^{\text {th }}\right.$ ed.). Boston: Allyn \& Bacon.

Tannenbaum, S. (2002). A strategic view of organizational training and learning. In K. Kraiger (Ed.), Creating, implementing, and managing effective training and development (pp. 10-52). San Fransico, CA: Jossey-Bass.

Tannenbaum, S. I. \& Yukl, G. (1992). Training and development in work organizations. Annual Review of Psychology, 43, 399-441.

Taras, V., Kirkman, B. L., \& Steel, P. (2010). Examining the impact of culture's consequences: A three-decade, multilevel, meta-analytic review of Hofstede's cultural value dimensions. Journal of Applied Psychology, 95, 405. 
Responsive Training 165

Taylor, A. S., James, K., \& Murry, A. (2012). Leveraging workforce diversity using a multidimensional approach. In C. L. Scott \& M. Y. Byrd (Eds.), Handbook of Research on Workforce Diversity and in a Global Society: Technologies and Concepts (pp. 203-224). Hershey, PA: IGI Global.

Taylor, P. J., Russ-Eft, D. F., \& Chan, D. W. (2005). A meta-analytic review of behavior modeling training. Journal of Applied Psychology, 90, 692.

Tharp, R. G. (1982). The effective instruction of comprehension: Results and description of the Kamehameha Early Education Program. Reading Research Quarterly, 503-527.

Tharp, R. G. (1989). Psychocultural variables and constants: Effects on teaching and learning in schools (Vol. 44, No. 2, p. 349). American Psychological Association.

Tharp, R. G., Jordan, C., Speidel, G. E., Hu-Pei Au, K., Klein, T. W., Calkins, R. P., ... \& Gallimore, R. (2007). Education and native Hawaiian children: Revisiting KEEP. Hũlili: Multidisciplinary Research on Hawaiian Well-Being, 4, 269-317.

Tharp, R.G. \& Gillmore, R. (1989). Rousing minds to life: Teaching and learning in social context. New York, NY: Cambridge University Press.

Tharp, R.G., Estrada, P., Dalton, S.S., \& Yamauchi, L.A. (2000). Teaching transformed: Achieving excellence, fairness, inclusion and harmony. Boulder, CO: Westview Press.

Tharp, R.G. \& Yamauchi, L.A. (1994). Effective instructional conversation in Native American classrooms. Educational practice report. National Center for Research on Cultural Diversity and Second Language Learning. Washington DC: Office of Educational Research and Improvement.

Thornton, R. (1987). American Indian holocaust and survival: A population history since 
1492 (Vol. 186). University of Oklahoma Press.

Thornton, R. (1997). Tribal membership requirements and the demography of 'old'and 'new'Native Americans. Population Research and Policy Review, 16, 33-42.

Tisdell, E. J., Taylor, E. W., \& Forté, K. S. (2013). Community-Based Financial Literacy Education in a Cultural Context A Study of Teacher Beliefs and Pedagogical Practice. Adult Education Quarterly, 63, 338-356.

Tomlinson, C. A. (2000). Reconcilable differences: Standards-based teaching and differentiation. Educational Leadership, 58, 6-13.

Tracey, J.B., Tannenbaum, S.I., \& Kavanagh, M.J. (1995). Applying trained skills on the job: The importance of the work environment. Journal of Applied Psychology, 80, 239-252.

Trennert, R. A. (1982). Educating Indian girls at nonreservation boarding schools, 1878 1920. The Western Historical Quarterly, 271-290.

Tsuji, L. J., \& Ho, E. (2002). Traditional environmental knowledge and western science: in search of common ground. Canadian Journal of Native Studies, 22, 327-360.

Tung, R. L. (1981). Selection and training of personnel for overseas assignments. Columbia Journal of World Business, 16, 68-78.

Turner, K. M., Richards, M., \& Sanders, M. R. (2007). Randomised clinical trial of a group parent education programme for Australian Indigenous families. Journal of Paediatrics and Child Health, 43, 429-437.

United States Commission on Civil Rights. (2003). A quiet crisis: Federal funding and unmet needs in Indian country. Washington, DC: Office of Civil Rights Evaluation. 
Retrieved June 12th, 2014 from http://www2.ed.gov/about/reports/annual/ocr/reportto-president-2009-12.pdf.

United State Department of Education Office of Civil Rights [OCR]. (2012). Helping to ensure equal access to education: Report to the President and Secretary of education, under section 203(b)(1) of the Department of Education Organization Act, FY 2009 2012. Washington DC.

US Census. (2008). American Indian and Alaska Native Policy of the US Census Bureau. Retrieved June 1, 2014, from http://www.census.gov/aian/pdf/Appendix-F.pdf.

Van den Bussche, E., Van den Noortgate, W., \& Reynvoet, B. (2009). Mechanisms of masked priming: a meta-analysis. Psychological Bulletin, 135, 452.

Van Iddekinge, C.H., Ferris, G.R., Perrewé, P.L., Perryman, A.A., Blass, F.R., \& Heetderks, T.D. (2009). Effects of selection and training on unit-level performance over time: A latent growth modeling approach. Journal of Applied Psychology, 94, $829-843$.

Varma, S. (2008). Preliminary item statistics using point-biserial correlation and pvalues. Accessed Setempber 24th, 2014 from http://www.eddata.com/resources/publications/eds_point_biserial.pdf.

Villegas, A. M. (1991). Culturally Responsive Pedagogy for the 1990s and Beyond. Trends and Issues Paper No. 6. Washington DC: ERIC Clearinghouse on Teacher Education.

Villegas, A. M., \& Lucas, T. (2002). Preparing culturally responsive teachers rethinking the curriculum. Journal of Teacher Education, 53(1), 20-32. 
Vroom, V. (1964). Work and motivation. New York: John Wiley.

Walch, M. C. (1983). Terminating the Indian termination policy. Stanford Law Review, 1181-1215.

Waldman, C. (2009). Encyclopedia of Native American Tribes. Infobase Publishing.

Walker, D. M. (1995). Connecting Right and Left Brain: Increasing Academic Performance of African American Students through the Arts. Paper presented at the annual meeting of the National Alliance of Black School Educators, Dallas, November 15 th.

Walters, K. L. (1999). Urban American Indian identity attitudes and acculturation styles. Journal of Human Behavior in the Social Environment, 2, 163-178.

Wang, M. \& Takeuchi, R. (2007). The role of goal orientation during expatriation: a cross-sectional and longitudinal investigation. Journal of Applied Psychology, 92, 1437.

Wasti, S.A. (2003a). The influence of cultural values on antecedents of organisational commitment: an individual-level analysis. Applied Psychology, 52, 533-554.

Wasti, S.A. (2003b). Organizational commitment, turnover intentions and the influence of cultural values. Journal of Occupational and Organizational Psychology, 76, 303321.

Watahomigie, L. J., \& McCarty, T. L. (1994). Bilingual/bicultural education at Peach Springs: A Hualapai way of schooling. Peabody Journal of Education, 69, 26-42. Watahomigie, L. J., \& McCarty, T. L. (1994). Bilingual/bicultural education at Peach Springs: A Hualapai way of schooling. Peabody Journal of Education, 69, 26-42. 
Wexley, K. N. (1984). Personnel training. Annual Review of Psychology, 35(1), 519-551.

Whitbeck, L. B., Hoyt, D. R., McMorris, B. J., Chen, X., \& Stubben, J. D. (2001).

Perceived discrimination and early substance abuse among American Indian children. Journal of Health and Social Behavior, 42, 405-424.

Whitbeck, L. B., McMorris, B. J., Hoyt, D. R., Stubben, J. D., \& LaFromboise, T. (2002). Perceived discrimination, traditional practices, and depressive symptoms among American Indians in the upper Midwest. Journal of Health and Social Behavior, 43, 400-418.

Wight, A. R., Hammons, M. A., Bing, J. W., Center for Research and Education, \& the US Peace Corps. (1969). A Draft handbook for cross-cultural and community involvement training. Estes Park, CO: Center for Research and Education.

Williams, K. Y. \& O'Reilly, C. A. (1998). Demography and diversity in organizations: A review of 40 years of research. Research in Organizational Behavior, 20, 77-140.

Wood, P. B., \& Clay, W. C. (1996). Perceived structural barriers and academic performance among American Indian high school students. Youth \& Society, 28(1), 40-61.

World's Scientific Academies (2000). Transition to sustainability in the $21^{\text {st }}$ century: The contribution of science and technology. Retrieved April, 25, 2010 from http://www.interacademies.net/?id=3568.

Wright, S.C., Taylor, D., \& Macarthur, J. (2000). Subtractive bilingualism and the survival of the Inuit language: Heritage versus second-language education. Journal of Educational Psychology, 92, 63-84. 
Yardley, W. (March 25, 2011). Catholic order reaches \$166 million settlement with sexual abuse victims. The New York Times online. Retrieved May 12, 2012 from http://www.nytimes.com/2011/03/26/us/26jesuits.html?_r=0.

Young, E. (2010). Challenges to conceptualizing and actualizing culturally relevant pedagogy: How viable is the theory in classroom practice?. Journal of Teacher Education, 61, 248-260.

Zaniboni, S., Fraccaroli, F., Truxillo, D.M., Bertolino, M., \& Bauer, T.N. (2011). Training valence, instrumentality, and expectancy scale (T-VIES-it): Factor structure and nomological network in an Italian sample. Journal of Workplace Learning, 23, $133-151$.

Ziibiwing Center (2011). American Indian boarding schools: An exploration of global ethnic \& cultural cleansing. Retrieved June 13, 2014 from http://www.sagchip.org/ziibiwing/planyourvisit/pdf/aibscurrguide.pdf. 


\section{APPENDIX A: Manipulation check (video prime evaluation)}

While you are watching this video, please provide your feedback on the following items. Quality

1. How would you rate the audio quality?

\begin{tabular}{|c|c|c|c|}
\hline Poor & Okay & Good & Very good \\
\hline$\square$ & $\square$ & $\square$ & $\square$ \\
\hline
\end{tabular}

2. How would you rate the video quality?

\begin{tabular}{|c|c|c|c|}
\hline Poor & Okay & Good & Very good \\
\hline$\square$ & $\square$ & $\square$ & $\square$ \\
\hline
\end{tabular}

3. How would you rate the pace of this video?

\begin{tabular}{|c|c|c|c|}
\hline Too slow & Okay & Good & Too Fast \\
\hline$\square$ & $\square$ & $\square$ & $\square$ \\
\hline
\end{tabular}

\section{Content}

4. How clear is the message in this video?

\begin{tabular}{|c|c|c|c|}
\hline Not clear & A little & Clear & Very much so \\
\hline$\square$ & $\square$ & $\square$ & $\square$ \\
\hline
\end{tabular}

5. How relevant is this video's message to the work you do with your community?

\begin{tabular}{|c|c|c|c|}
\hline Not relevant & A little & Relevant & Very much so \\
\hline$\square$ & $\square$ & $\square$ & $\square$ \\
\hline
\end{tabular}

6. How culturally appropriate is this video to your community?

Not appropriate A little Appropriate Very much so

7. What would you say is the underlying message of this video? 


\section{APPENDIX B: Time 1 (pre-training) survey}

\section{Please complete the questions below.}

1. What type of work do you do?

What is your job title?

2. What is your age (how old are you)?

3. What is your self-identified gender?

a. Female

b. Male

c. Other

4. What is your household's average annual income?

$\begin{array}{ccccccc}<\$ 10,000 & \$ 10- & \$ 20- & \$ 30- & \$ 40- & \$ 50- & \$ 60,000+ \\ & 20,000 & 30,000 & 40,000 & 50,000 & 60,000 & \end{array}$

5. What is (are) your ethnicity(ies)? (You can check more than one if necessary)

a. Native American

b. White

c. Black

d. Latino

e. Asian

f. Other

6. If you checked "Native American," what is/are your tribal affiliation(s)?

7. What level of education do you have so far?
a. Doctoral degree
b. Master's degree
c. Bachelor degree
d. Associate degree
e. High school diploma/GED
f. Less than high school

8. Do you live by or follow:

A lot Some A little $\begin{gathered}\text { Not at } \\ \text { all }\end{gathered}$

A Native American way of life A White way of life An African-American way of life A Latino/a way of life 
An Asian way of life Another way of life

9. Does your family live by or follow:

A lot Some A little Not at

A Native American way of life A White way of life An African-American way of life A Latino/a way of life An Asian way of life Another way of life

10. Are you successful in:

\section{A lot Some A little Not at}

A Native American way of life A White way of life An African-American way of life A Latino/a way of life An Asian way of life Another way of life

11. Is your mother successful in:

A lot Some A little Not at

A Native American way of life A White way of life An African-American way of life A Latino/a way of life An Asian way of life Another way of life

12. Is your father successful in:

A lot Some A little Not at

A Native American way of life A White way of life An African-American way of life A Latino/a way of life An Asian way of life Another way of life

$\begin{array}{ll}\square & \square \\ \square & \square \\ \square & \square \\ \square & \square \\ \square & \square \\ \square & \square\end{array}$

all

3. Some families have special activities or traditions that take place every year at particular times. How many did your family have when you were growing up that were based on:

A lot Some A little $\begin{gathered}\text { Not at } \\ \text { all }\end{gathered}$


A Native American way of life

A White way of life

An African-American way of life

A Latino/a way of life

An Asian way of life

Another way of life

$\begin{array}{llll}\square & \square & \square & \square \\ \square & \square & \square & \square \\ \square & \square & \square & \square \\ \square & \square & \square & \square \\ \square & \square & \square & \square \\ \square & \square & \square & \square\end{array}$

14. How many special activities or traditions do you participate in based on:

A lot Some A little Not at

A Native American way of life

A White way of life

An African-American way of life

A Latino/a way of life

An Asian way of life

Another way of life

15. How much experience have you had conducting research?
None
A little
Some
Quite a bit
A lot

16. How often do you use research for the work you do?
None
A little
Some
Quite a bit
A lot

17. "My role at work allows me to be involved in research"

Strongly

Not much

Neutral

Agree

all

disagree

The questions below are an assessment, not a test.

18. Some things are studied scientifically; some things are studied in other ways. Would you say you have (circle one):

a. A clear understanding of what it means to study something scientifically,

b. A general sense of what it means, or

c. No understanding of its meaning?

19. From your point of view, what does it mean to study something scientifically? (Just in your own words)

20. Now, please think of this situation. Two scientists want to know if a certain drug is effective against high blood pressure. The first scientist wants to give the drug to 1,000 people with high blood pressure levels. The second scientist wants to give the drug to 500 people with high blood pressure, and not give the drug to 
another 500 people with high blood pressure, and see how many in both groups experience lower blood pressure levels. Which is the better way to test this drug? The first way

The second way

21. Why is it better to test the drug this way?

22. A doctor tells a couple that their genetic make-up means that they've got a one-infour chance of having a child with an inherited illness. Please indicate whether each of four statements is a correct or incorrect interpretation of the meaning of "one-in-four chances":

If they have only three children, none will have the illness

If their first child has the illness, the next three will not

Each of the couple's children has the same risk of suffering from the illness

If their first three children are healthy, the fourth will have the illness

Correct Incorrect

23. What is the difference between a population and a sample?

a. There is no difference.

b. A population is everybody in a category of interest, while a sample only includes the people in my study.

c. A population is everybody in a country, while a sample is only people in my area.

d. I don't know.

24. SAT scores are an example of what kind of data?
a. Continuous
b. Ordinal
c. Dichotomous
d. Nominal
e. I don't know.

25. What is the difference between reliability and validity?

a. Reliability is how consistent my measurements are, while validity is whether or not I am are measuring the right thing.

b. Reliability means my findings are stable, while validity does not.

c. Reliability and validity are the same.

d. I don't know.

26. What does it mean if a research finding is "confounded"?

a. It means the relationship between my measurements is complicated.

b. It doesn't mean anything.

c. It means my results could be caused by things I did not account for.

d. I don't know.

27. What is the benefit to experimental research versus correlational research? 
a. There are no benefits of experimental research over correlational research.

b. Correlational research looks at relationships between things.

c. Experiments can establish cause and effect.

d. I don't know.

28. What is the difference between a variable and a construct?

a. They are the same.

b. A variable varies while a construct stays the same.

c. A variable is a measured expression of a construct.

d. I don't know.

29. What is an independent variable?

a. I don't know.

b. The variable that the experimenter manipulates to determine its effect.

c. A variable that is separate, or independent, of the project.

d. It is a variable that has its own stable income.

30. What is a dependent variable?

a. It is the variable that we expect to change depending on some other variable.

b. It is a quantitative variable.

c. I don't know.

d. It is a variable that is not living on their own.

31. What is the difference between "basic" and "applied" research?

a. Basic research is simpler.

b. Basic is for knowledge sake, while applied attempts to solve real-world problems.

c. Basic research and applied research are the same.

d. I don't know.

32. Below is a table of disciplinary action (in school) per ethnic group in the state of Oregon.

\begin{tabular}{|c|c|}
\hline Ethnicity & $\begin{array}{c}\text { Percent of students with } \\
\text { one or more discipline } \\
\text { incidents } \\
\text { (2013-2014) }\end{array}$ \\
\hline Asian & 1.7 \\
\hline Black/African-American & 11 \\
\hline Hispanic/Latino & 7.2 \\
\hline $\begin{array}{c}\text { American Indian/Alaskan } \\
\text { Native }\end{array}$ & 10.3 \\
\hline Multi-racial & 6.2 \\
\hline $\begin{array}{c}\text { Native Hawaiian/Pacific } \\
\text { Islander }\end{array}$ & 6.5 \\
\hline White & 5.4 \\
\hline
\end{tabular}

a. What is the average (i.e., mean) percentage of students with one or more disciplinary incidents (using rounded numbers is okay)?

b. Please draw a graph that shows the proportion of students above and below average: 
Responsive Training 177

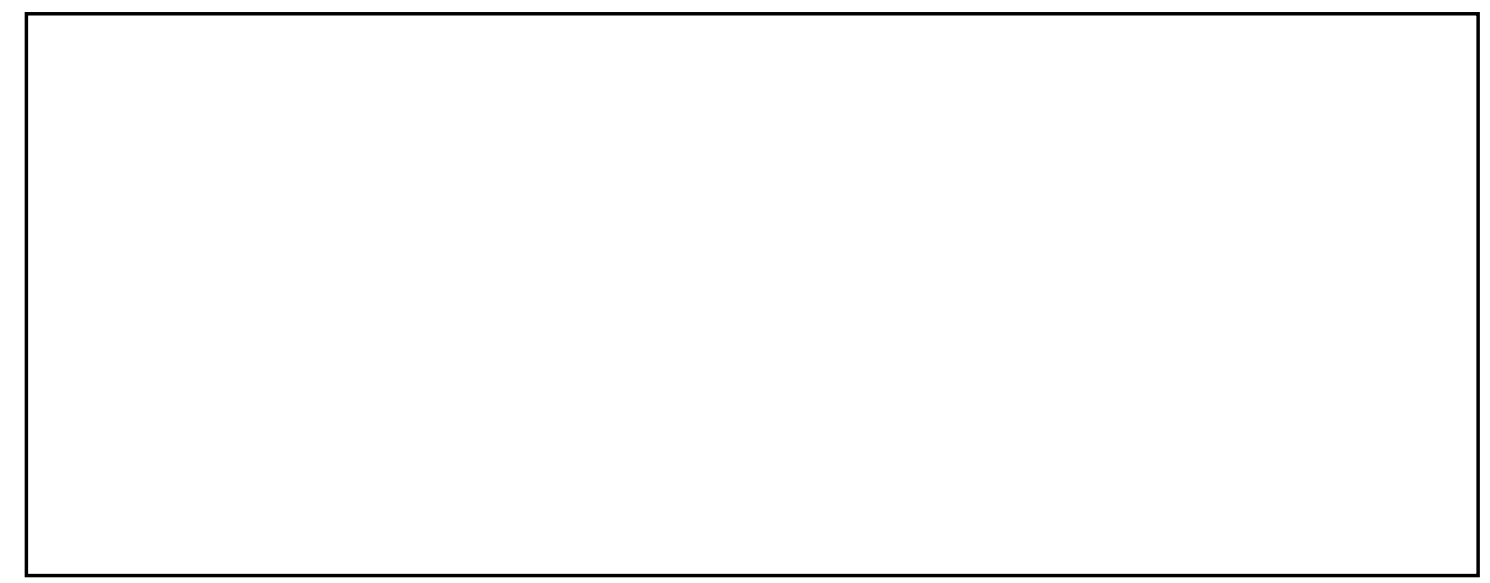

a. What is (are) the independent variable(s)?

b. What is the dependent variable?

c. What does this graph tell us about American Indian students? 


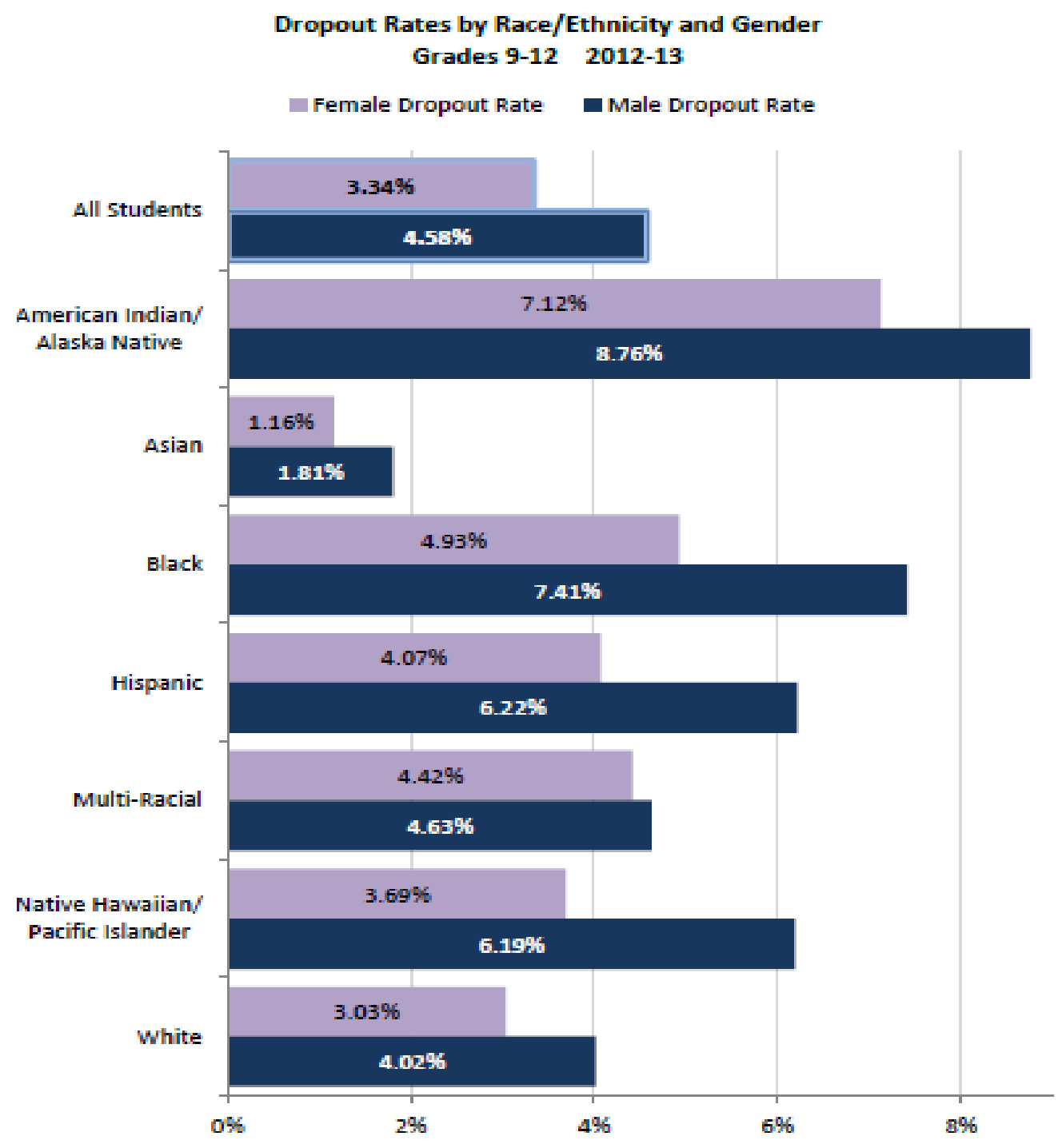

\begin{tabular}{|l|c|c|c|c|c|}
\hline Research as a goal & $\begin{array}{l}\text { Strongly } \\
\text { disagree }\end{array}$ & Disagree & $\begin{array}{l}\text { Neither } \\
\text { agree or } \\
\text { disagree }\end{array}$ & Agree & $\begin{array}{l}\text { Strongly } \\
\text { agree }\end{array}$ \\
\hline $\begin{array}{l}\text { 34. It's hard to take the goal of } \\
\text { conducting research seriously. }\end{array}$ & $\square$ & $\square$ & $\square$ & $\square$ & $\square$ \\
\hline $\begin{array}{l}\text { 35. Quite frankly, I don't care if I am } \\
\text { able to conduct research or not. }\end{array}$ & $\square$ & $\square$ & $\square$ & $\square$ & $\square$ \\
\hline $\begin{array}{l}\text { 36. I am strongly committed to } \\
\text { pursuing research skills. }\end{array}$ & $\square$ & $\square$ & $\square$ & $\square$ & $\square$ \\
\hline $\begin{array}{l}\text { 37. It wouldn't take much to make } \\
\text { me abandon acquiring research skills } \\
\text { as a goal. }\end{array}$ & $\square$ & $\square$ & $\square$ & $\square$ & $\square$ \\
\hline 38. I think learning to understand & $\square$ & $\square$ & $\square$ & $\square$ & $\square$ \\
\hline
\end{tabular}


Responsive Training 179

\begin{tabular}{|c|c|c|c|c|c|}
\hline research is a good goal to shoot for. & & & & & \\
\hline Research and me & $\begin{array}{l}\text { Strongly } \\
\text { disagree }\end{array}$ & Disagree & $\begin{array}{l}\text { Neither } \\
\text { agree or } \\
\text { disagree }\end{array}$ & Agree & $\begin{array}{l}\text { Strongly } \\
\text { agree }\end{array}$ \\
\hline $\begin{array}{l}\text { 39. People like me belong in } \\
\text { research. }\end{array}$ & $\square$ & $\square$ & $\square$ & $\square$ & $\square$ \\
\hline $\begin{array}{l}\text { 40. My ethnic/racial group is good at } \\
\text { conducting research. }\end{array}$ & $\square$ & $\square$ & $\square$ & $\square$ & $\square$ \\
\hline $\begin{array}{l}\text { 41. People my age are better at using } \\
\text { data than people of other ages. }\end{array}$ & $\square$ & $\square$ & $\square$ & $\square$ & $\square$ \\
\hline $\begin{array}{l}\text { 42. Doing research is important to my } \\
\text { self-image. }\end{array}$ & $\square$ & $\square$ & $\square$ & $\square$ & $\square$ \\
\hline $\begin{array}{l}\text { 43. I regret having entered into a field } \\
\text { that involves research. }\end{array}$ & $\square$ & $\square$ & $\square$ & $\square$ & $\square$ \\
\hline $\begin{array}{l}\text { 44. I am proud to be in a profession } \\
\text { that involves research. }\end{array}$ & $\square$ & $\square$ & $\square$ & $\square$ & $\square$ \\
\hline $\begin{array}{l}\text { 45. I dislike the idea of being a } \\
\text { researcher. }\end{array}$ & $\square$ & $\square$ & $\square$ & $\square$ & $\square$ \\
\hline $\begin{array}{l}\text { 46. I do not identify with the research } \\
\text { profession. }\end{array}$ & $\square$ & $\square$ & $\square$ & $\square$ & $\square$ \\
\hline 47. I am enthusiastic about research. & $\square$ & $\square$ & $\square$ & $\square$ & $\square$ \\
\hline I am confident that I can... & $\begin{array}{l}\text { Strongly } \\
\text { disagree }\end{array}$ & Disagree & $\begin{array}{l}\text { Neither } \\
\text { agree or } \\
\text { disagree }\end{array}$ & Agree & $\begin{array}{l}\text { Strongly } \\
\text { agree }\end{array}$ \\
\hline 48. Generate researchable questions & $\square$ & $\square$ & $\square$ & $\square$ & $\square$ \\
\hline $\begin{array}{l}\text { 49. Develop a logical rationale for my } \\
\text { particular research ideas }\end{array}$ & $\square$ & $\square$ & $\square$ & $\square$ & $\square$ \\
\hline $\begin{array}{l}\text { 50. Present my research idea orally or } \\
\text { in written form to a group }\end{array}$ & $\square$ & $\square$ & $\square$ & $\square$ & $\square$ \\
\hline 51. Discuss research ideas with peers & $\square$ & $\square$ & $\square$ & $\square$ & $\square$ \\
\hline $\begin{array}{l}\text { 52. Choose an appropriate research } \\
\text { design }\end{array}$ & $\square$ & $\square$ & $\square$ & $\square$ & $\square$ \\
\hline $\begin{array}{l}\text { 53. Participate in generating } \\
\text { collaborative research ideas }\end{array}$ & $\square$ & $\square$ & $\square$ & $\square$ & $\square$ \\
\hline $\begin{array}{l}\text { 54. Use an existing computer package } \\
\text { to analyze data }\end{array}$ & $\square$ & $\square$ & $\square$ & $\square$ & $\square$ \\
\hline $\begin{array}{l}\text { 55. Interpret and understand } \\
\text { statistical printouts }\end{array}$ & $\square$ & $\square$ & $\square$ & $\square$ & $\square$ \\
\hline $\begin{array}{l}\text { 56. Choose measures of dependent } \\
\text { and independent variables }\end{array}$ & $\square$ & $\square$ & $\square$ & $\square$ & $\square$ \\
\hline $\begin{array}{l}\text { 57. Use computer software to } \\
\text { generate graphics }\end{array}$ & $\square$ & $\square$ & $\square$ & $\square$ & $\square$ \\
\hline $\begin{array}{l}\text { 58. Organize collected data for } \\
\text { analysis }\end{array}$ & $\square$ & $\square$ & $\square$ & $\square$ & $\square$ \\
\hline 59. Report results in graphic form & $\square$ & $\square$ & $\square$ & $\square$ & $\square$ \\
\hline $\begin{array}{l}\text { 60. Choose methods of data } \\
\text { collection }\end{array}$ & $\square$ & $\square$ & $\square$ & $\square$ & $\square$ \\
\hline $\begin{array}{l}\text { 61. Orally present results to my } \\
\text { research group }\end{array}$ & $\square$ & $\square$ & $\square$ & $\square$ & $\square$ \\
\hline $\begin{array}{l}\text { 62. Identify implications for future } \\
\text { research }\end{array}$ & $\square$ & $\square$ & $\square$ & $\square$ & $\square$ \\
\hline
\end{tabular}


Responsive Training 180

\begin{tabular}{|c|c|c|c|c|c|}
\hline $\begin{array}{l}\text { 63. Identify and report limitations of } \\
\text { my research }\end{array}$ & $\square$ & $\square$ & $\square$ & $\square$ & $\square$ \\
\hline Training-specific & $\begin{array}{l}\text { Strongly } \\
\text { disagree }\end{array}$ & Disagree & $\begin{array}{l}\text { Neither } \\
\text { agree or } \\
\text { disagree }\end{array}$ & Agree & $\begin{array}{l}\text { Strongly } \\
\text { agree }\end{array}$ \\
\hline $\begin{array}{l}\text { 64. I want to improve my research } \\
\text { knowledge for my job by attending } \\
\text { this training. }\end{array}$ & $\square$ & $\square$ & $\square$ & $\square$ & $\square$ \\
\hline $\begin{array}{l}\text { 65. I feel that it is important to take } \\
\text { part in this training in order to } \\
\text { strengthen my research skills. }\end{array}$ & $\square$ & $\square$ & $\square$ & $\square$ & $\square$ \\
\hline $\begin{array}{l}\text { 66. I think it's important to learn new } \\
\text { things from this training. }\end{array}$ & $\square$ & $\square$ & $\square$ & $\square$ & $\square$ \\
\hline $\begin{array}{l}\text { 67. I believe this training is useful for } \\
\text { employees that occupy a job position } \\
\text { similar to mine. }\end{array}$ & $\square$ & $\square$ & $\square$ & $\square$ & $\square$ \\
\hline $\begin{array}{l}\text { 68. Usually I am able to apply what I } \\
\text { learn in training activities to my job. }\end{array}$ & $\square$ & $\square$ & $\square$ & $\square$ & $\square$ \\
\hline $\begin{array}{l}\text { 69. Acquiring new skills from this } \\
\text { training will positively influence my } \\
\text { performance on the job. }\end{array}$ & $\square$ & $\square$ & $\square$ & $\square$ & $\square$ \\
\hline $\begin{array}{l}\text { 70. If I am engaged in this training } \\
\text { activity, I am confident that I can } \\
\text { master research aspects of my job. }\end{array}$ & $\square$ & $\square$ & $\square$ & $\square$ & $\square$ \\
\hline $\begin{array}{l}\text { 71. If I am engaged in this training's } \\
\text { activities, I am confident to learn the } \\
\text { new knowledge taught in this } \\
\text { training. }\end{array}$ & $\square$ & $\square$ & $\square$ & $\square$ & $\square$ \\
\hline $\begin{array}{l}\text { 72. If I am engaged in this training } \\
\text { activity, I am confident I can improve } \\
\text { my ability to initiate research in my } \\
\text { job. }\end{array}$ & $\square$ & $\square$ & $\square$ & $\square$ & $\square$ \\
\hline
\end{tabular}

Done! 
Responsive Training 181

\section{APPENDIX C: Time 2 (post-training) survey}

1. Some things are studies scientifically; some things are studies in other ways. Would you say you have (circle one):

a. A clear understanding of what it means to study something scientifically,

b. A general sense of what it means, or

c. No understanding of its meaning?

2. From your point of view, what does it mean to study something scientifically? (Just in your own words)

3. Now, please think of this situation. Two scientists want to know if a certain drug is effective against high blood pressure. The first scientist wants to give the drug to 1,000 people with high blood pressure levels. The second scientist wants to give the drug to 500 people with high blood pressure, and not give the drug to another 500 people with high blood pressure, and see how many in both groups experience lower blood pressure levels. Which is the better way to test this drug?

The first way

The second way

4. Why is it the better to test the drug this way?

5. A doctor tells a couple that their genetic make-up means that they've got a one-infour chance of having a child with an inherited illness. Please indicate whether each of four statements is a correct or incorrect interpretation of the meaning of "one-in-four chances":

If they have only three children, none will have the illness

Correct Incorrect If their first child has the illness, the next three will not Each of the couple's children has the same risk of suffering from the illness

If their first three children are healthy, the fourth will have the illness

6. What is the difference between a population and a sample?

a. There is no difference.

b. A population is everybody in a category of interest, while a sample only includes the people in my study.

c. A population is everybody in a country, while a sample is only people in my area.

d. I don't know. 
7. SAT scores are an example of what kind of data?
a. Continuous
b. Ordinal
c. Dichotomous
d. Nominal
e. I don't know.

8. What is the difference between reliability and validity?

a. Reliability is how consistent my measurements are, while validity is whether or not I am are measuring the right thing.

b. Reliability means my findings are stable, while validity does not.

c. Reliability and validity are the same.

d. I don't know.

9. What does it mean if a research finding is "confounded"?

a. It means the relationship between my measurements is complicated.

b. It doesn't mean anything.

c. It means my results could be caused things I did not account for.

d. I don't know.

10. What is the benefit to experimental research versus correlational research?

a. There are no benefits of experimental research over correlational research.

b. Correlational research looks at relationships between things.

c. Experiments can establish cause and effect.

d. I don't know.

11. What is the difference between a variable and a construct?

a. They are the same.

b. A variable varies while a construct stays the same.

c. A variable is a measured expression of a construct.

d. I don't know.

12. What is an independent variable?

a. I don't know.

b. The variable that the experimenter manipulates to determine its effect.

c. A variable that is separate, or independent, of the project.

d. It is a variable that has its own stable income.

13. What is a dependent variable?

a. It is the variable that we expect to change depending on some other variable.

b. It is a quantitative variable.

c. I don't know.

d. It is a variable that is not living on their own.

14. What is the difference between "basic" and "applied" research?

a. Basic research is simpler.

b. Basic is for knowledge sake, while applied attempts to solve real-world problems.

c. Basic research and applied research are the same.

d. I don't know. 
15. Please look at the graph below.

a. What is (are) the independent variable(s)?

b. What is the dependent variable?

c. What does this graph tell us about American Indian students?

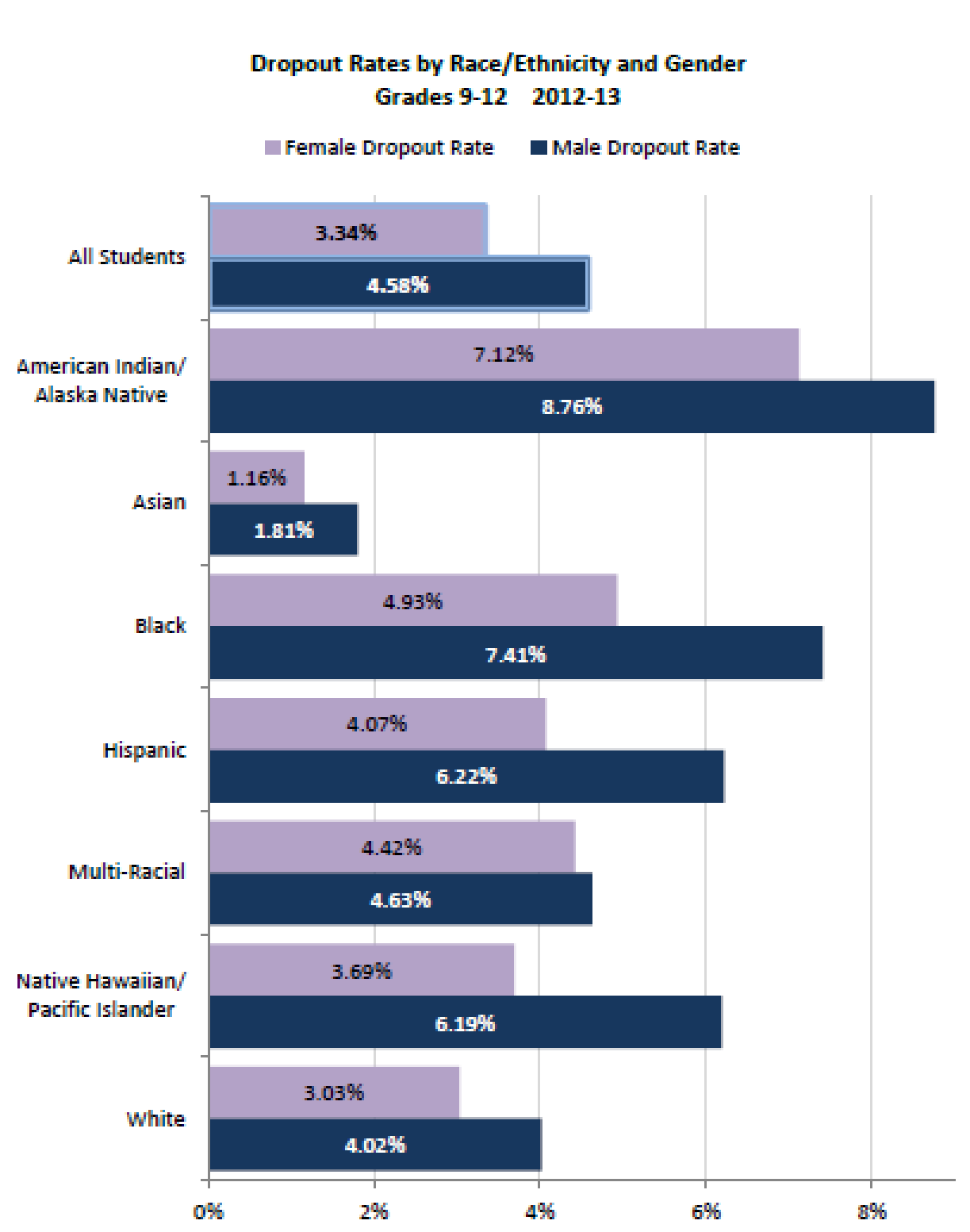


16. Below is a table of disciplinary action (in school) per ethnic group in the state of Oregon.

\begin{tabular}{|c|c|}
\hline Ethnicity & $\begin{array}{c}\text { Percent of students with one or more discipline incidents } \\
(\mathbf{2 0 1 3 - 2 0 1 4 )}\end{array}$ \\
\hline Asian & 1.7 \\
\hline Black/African-American & 11 \\
\hline Hispanic/Latino & 7.2 \\
\hline American Indian/Alaskan Native & 10.3 \\
\hline Multi-racial & 6.2 \\
\hline Native Hawaiin/Pacific Islander & 6.5 \\
\hline White & 5.4 \\
\hline
\end{tabular}

a. What is the average (i.e., mean) percentage of students with one or more disciplinary incidents (using rounded numbers is okay)?

b. Please draw a graph that shows the proportion of students above and below average:

Please consider each statement below and rate your agreement.

\begin{tabular}{|c|c|c|c|c|}
\hline $\begin{array}{l}\text { Strongly } \\
\text { disagree }\end{array}$ & Disagree & $\begin{array}{l}\text { Neither } \\
\text { agree or } \\
\text { disagree }\end{array}$ & Agree & $\begin{array}{l}\text { Strongly } \\
\text { agree }\end{array}$ \\
\hline$\square$ & $\square$ & $\square$ & $\square$ & $\square$ \\
\hline$\square$ & $\square$ & $\square$ & $\square$ & $\square$ \\
\hline$\square$ & $\square$ & $\square$ & $\square$ & $\square$ \\
\hline$\square$ & $\square$ & $\square$ & $\square$ & $\square$ \\
\hline$\square$ & $\square$ & $\square$ & $\square$ & $\square$ \\
\hline $\begin{array}{l}\text { Strongly } \\
\text { disagree }\end{array}$ & Disagree & $\begin{array}{l}\text { Neither } \\
\text { agree or } \\
\text { disagree }\end{array}$ & Agree & $\begin{array}{l}\text { Strongly } \\
\text { agree }\end{array}$ \\
\hline$\square$ & $\square$ & $\square$ & $\square$ & $\square$ \\
\hline$\square$ & $\square$ & $\square$ & $\square$ & $\square$ \\
\hline$\square$ & $\square$ & $\square$ & $\square$ & $\square$ \\
\hline
\end{tabular}

17. It's hard to take the goal of conducting research seriously. 18. Quite frankly, I don't care if I am able to conduct research or not. 19. I am strongly committed to pursuing research skills.

20. It wouldn't take much to make me abandon acquiring research skills as a goal.

21. I think learning to understand research is a good goal to shoot for.

I am confident that I can...

21. Generate researchable questions 22. Develop a logical rationale for my particular research ideas

23. Present my research idea orally or

\section{Strongly}

disagree

agree or

disagree

agree or sagree 
in written form to a group

24. Discuss research ideas with peers

25. Choose an appropriate research

design

26. Participate in generating

collaborative research ideas

27. Use an existing computer package to analyze data

28. Interpret and understand

statistical printouts

29. Choose measures of dependent

and independent variables

30 . Use computer software to generate graphics

31. Organize collected data for analysis

32. Report results in graphic form

33. Choose methods of data

collection

34. Orally present results to my

research group

35. Identify implications for future research

36. Identify and report limitations of my research

Please consider each statement below and rate your agreement.

$\begin{array}{lll}\begin{array}{l}\text { Strongly } \\ \text { disagree }\end{array} & \text { Disagree } & \begin{array}{l}\text { Neither } \\ \text { agree or } \\ \text { disagree }\end{array}\end{array}$

Agree Strongly

agree

37. People like me belong in

research.

38. My ethnic/racial group is good at conducting research.

39. People my age are better at using data than people of other ages.

40. Doing research is important to my self-image.

41. I regret having entered into a field that involves research.

42. I am proud to be in a profession that involves research.

43. I dislike the idea of being a researcher.

44. I do not identify with the research profession.

45. I am enthusiastic about research.

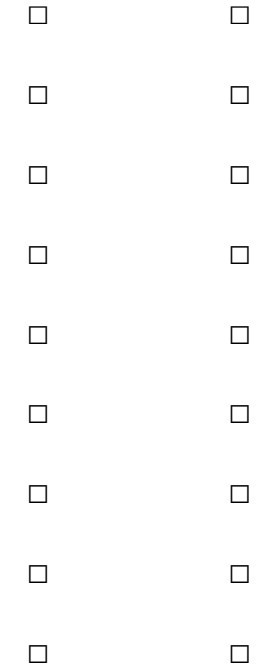

Overall Evaluation

Did you find this training useful? How? 
Responsive Training 186

How might this training be improved?

1. The content of this training was relevant to my cultural community.
a. Strongly disagree
b. Disagree
c. Neither agree or disagree
d. Agree
e. Strongly agree

2. The content of this training was culturally appropriate.
a. Strongly disagree
b. Disagree
c. Neither agree or disagree
d. Agree
e. Strongly agree

3. The pace of this training was culturally appropriate.
a. Strongly disagree
b. Disagree
c. Neither agree or disagree
d. Agree
e. Strongly agree

4. Ideas were communicated to me in a way I felt comfortable with.
a. Strongly disagree
b. Disagree
c. Neither agree or disagree
d. Agree
e. Strongly agree

5. I felt as though my ethnic identity was supported throughout the training.
a. Strongly disagree
b. Disagree
c. Neither agree or disagree
d. Agree
e. Strongly agree 
Would you be interested in any of the following workshops if we were to extend the program?

\begin{tabular}{|l|c|c|}
\hline & Interested & Not interested \\
\hline Where to find data sources? & $\square$ & $\square$ \\
\hline Qualitative research & $\square$ & $\square$ \\
\hline Statistical analyses & $\square$ & $\square$ \\
\hline Mixed method research & $\square$ & $\square$ \\
\hline $\begin{array}{l}\text { Culturally responsive } \\
\text { approaches }\end{array}$ & $\square$ & $\square$ \\
\hline $\begin{array}{l}\text { Research issues in Indian } \\
\text { Country }\end{array}$ & $\square$ & \\
\hline
\end{tabular}

Thank You!

We would like to follow up with you in the future with regard to this training. If that is okay with you, please provide an email that we can reach you at below: 


\section{APPENDIX D: Code in " $R$ ” for Hypothesis Testing}

Model 1: Hypothesized variables only

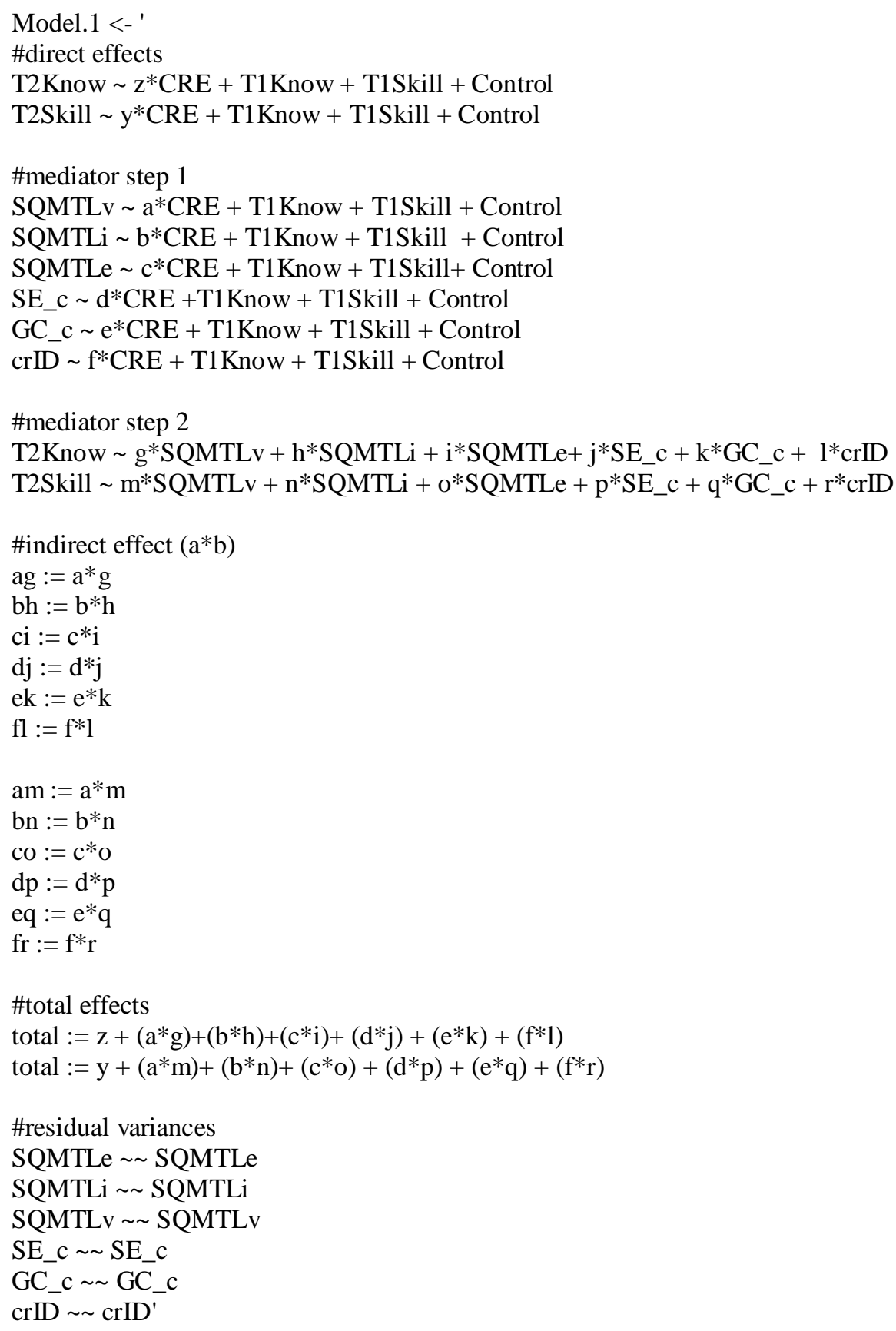


Responsive Training 189

\section{Model 2: Covariate model}

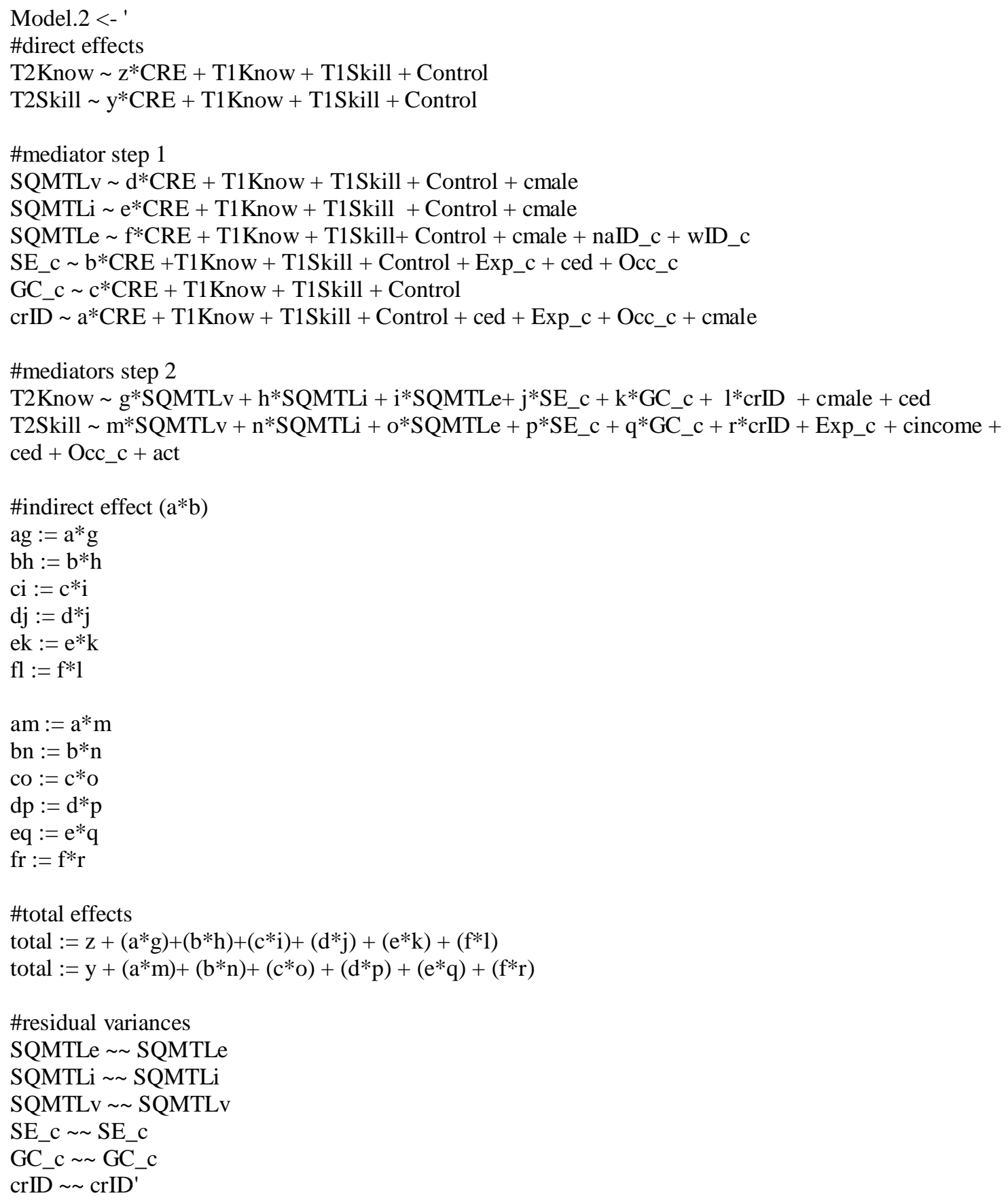

